Southern Illinois University Carbondale

OpenSIUC

Articles and Preprints

Department of Mathematics

1998

\title{
Stochastic Differential Systems with Memory: Theory, Examples and Applications
}

Salah-Eldin A. Mohammed

Southern Illinois University Carbondale, salah@sfde.math.siu.edu

Follow this and additional works at: http://opensiuc.lib.siu.edu/math_articles

For Proceedings of the Sixth Oslo-Silivri Workshop, Geilo, Norway, July 29-August 4, 1996 (Invited presentation of six hourly lectures), Published in Stochastic Analysis and Related Topics VI. The Geilo Workshop, 1996, Progress in Probability, vol. 42; ed. L. Decreusefond, Jon Gjerde, B. Oksendal, A.S. Ustunel; Birkhauser (1998), 1-77. The original publication is available at www.springerlink.com. Presentation slides are posted at http://opensiuc.lib.siu.edu/math_misc/28/.

\section{Recommended Citation}

Mohammed, Salah-Eldin A. "Stochastic Differential Systems with Memory: Theory, Examples and Applications." (Jan 1998).

This Article is brought to you for free and open access by the Department of Mathematics at OpenSIUC. It has been accepted for inclusion in Articles and Preprints by an authorized administrator of OpenSIUC. For more information, please contact opensiuc@lib.siu.edu. 


\title{
STOCHASTIC DIFFERENTIAL SYSTEMS WITH MEMORY. THEORY, EXAMPLES AND APPLICATIONS
}

\author{
Salah-Eldin A. Mohammed ${ }^{\dagger}$ \\ Department of Mathematics \\ Southern Illinois University \\ Carbondale, IL 62901-4408 USA \\ Email: salah@math.siu.edu \\ Web page: http://sfde.math.siu.edu
}

Stochastic Analysis and Related Topics VI. The Geilo Workshop, 1996, ed. L. Decreusefond, Jon Gjerde, B. Oksendal, A.S. Ustunel, Progress in Probability, Birkhauser (1998).

${ }^{\dagger}$ Research supported in part by NSF Grants DMS-9206785 and DMS-9503702. 


\section{Introduction}

The purpose of this article is to introduce the reader to certain aspects of stochastic differential systems, whose evolution depends on the past history of the state.

Chapter I begins with simple motivating examples. These include the noisy feedback loop, the logistic time-lag model with Gaussian noise, and the classical "heat-bath" model of $R$. Kubo, modeling the motion of a "large" molecule in a viscous fluid. These examples are embedded in a general class of stochastic functional differential equations (sfde's). We then establish pathwise existence and uniqueness of solutions to these classes of sfde's under local Lipschitz and linear growth hypotheses on the coefficients. It is interesting to note that the above class of sfde's is not covered by classical results of Protter, Metivier and Pellaumail and Doleans-Dade.

In Chapter II, we prove that the Markov (Feller) property holds for the trajectory random field of a sfde. The trajectory Markov semigroup is not strongly continuous for positive delays, and its domain of strong continuity does not contain tame (or cylinder) functions with evaluations away from 0 . To overcome this difficulty, we introduce a class of quasitame functions. These belong to the domain of the weak infinitesimal generator, are weakly dense in the underlying space of continuous functions and generate the Borel $\sigma$-algebra of the state space. This chapter also contains a derivation of a formula for the weak infinitesimal generator of the semigroup for sufficiently regular functions, and for a large class of quasitame functions.

In Chapter III, we study pathwise regularity of the trajectory random field in the time variable and in the initial path. Of note here is the non-existence of the stochastic flow for the singular sdde $d x(t)=x(t-r) d W(t)$ and a breakdown of linearity and local boundedness. This phenomenon is peculiar to stochastic delay equations. It leads naturally to a classification of sfde's into regular and singular types. Necessary and sufficient conditions for regularity are not known. The rest of Chapter III is devoted to results on sufficient conditions for regularity of linear systems driven by white noise or semimartingales, and Sussman-Doss type nonlinear sfde's.

Building on the existence of a compacting stochastic flow, we develop a multiplicative ergodic theory for regular linear sfde's driven by white noise, or general helix

semimartingales (Chapter IV). In particular, we prove a Stable Manifold Theorem for such systems. 
In Chapter V, we seek asymptotic stability for various examples of one-dimensional linear sfde's. Our approach is to obtain upper and lower estimates for the top Lyapunov exponent.

Several topics are discussed in Chapter VI. These include the existence of smooth densities for solutions of sfde's using the Malliavin calculus, an approximation technique for multidimensional diffusions using sdde's with small delays, and affine sfde's.

\section{Acknowledgments.}

This article is based on a series of six lectures delivered by the author to The Sixth Workshop on Stochastic Analysis, held at Geilo, Norway, July 29-August 4, 1996. The author is very grateful to the organizers for the hospitality and financial support.

The author wishes to thank Bernt Øksendal, Jorge Salazar and Tusheng Zhang for useful discussions. 


\section{Table of Contents}

I Existence................................................ 5

1 Examples and motivation............................... 5

2 General formulation. Existence and uniqueness.................. 12

$3 \quad$ Remarks and generalizations.............................. 18

II Markov Behavior and the Generator ............................ 19

1 Difficulties ......................................... 19

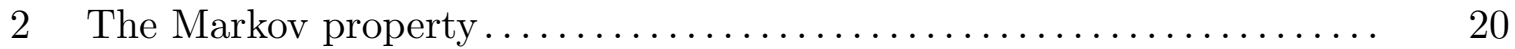

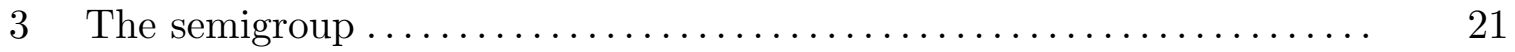

4 The weak infinitesimal generator........................... 24

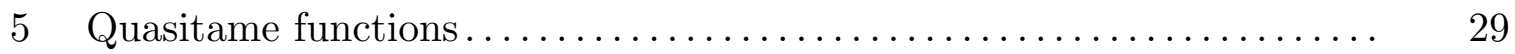

III Regularity and Classification of SFDE's .......................... 31

1 Measurable versions and regularity in distribution............... 31

2 Erratic behavior. The noisy feedback loop revisited .............. 34

3 Regularity of linear systems. White noise ................... 39

4 Regularity of linear systems. Semimartingale noise ............... 40

$5 \quad$ Regular nonlinear systems ............................... 42

IV Ergodic Theory of Linear SFDE's .............................. 45

1 Regular linear systems driven by white noise ................... 45

2 Regular linear systems driven by helix noise.................. 58

V Stability. Examples and Case Studies ........................... 62

1 The noisy feedback loop revisited once more $\ldots \ldots \ldots \ldots \ldots \ldots \ldots \ldots . \ldots 2$

2 Regular one-dimensional linear sfde's $\ldots \ldots \ldots \ldots \ldots \ldots \ldots \ldots \ldots \ldots, 68$

3 An sdde with Poisson noise .............................. 74

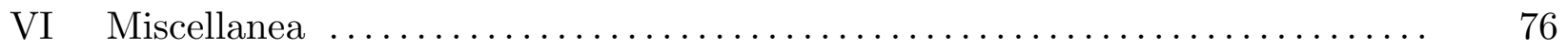

1 Malliavin calculus of sfde's.............................. 76

2 Diffusions via sdde's ................................... 82

3 Affine sfde's. A simple model of population growth............... 84

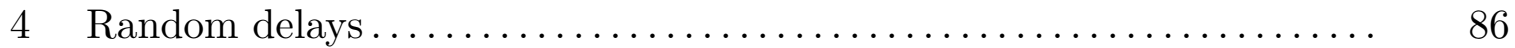

$5 \quad$ Infinite delays. Stationary solutions $\ldots \ldots \ldots \ldots \ldots \ldots \ldots \ldots \ldots \ldots . \ldots 6$

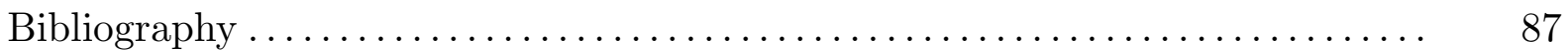




\section{Chapter I}

\section{Existence}

In this chapter we introduce several motivating examples of stochastic differential equations with memory. These simple examples include the the noisy feedback loop described by the stochastic differential delay equation (sdde)

$$
d x(t)=x(t-r) d W(t)
$$

driven by one-dimensional Brownian motion $W$, the logistic time-lag model with Gaussian noise

$$
d x(t)=[\alpha-\beta x(t-r)] x(t) d t+\sigma x(t) d W(t),
$$

and the classical "heat-bath" model proposed by R. Kubo $([\mathrm{Ku}])$ in order to model the motion of a large molecule in a viscous fluid.

We will formulate these physical models as stochastic functional differential equations (sfde's) with the appropriate choice of underlying state space. Our formulation leads to pathwise existence and uniqueness of solutions to the sfde. The existence theorem allows for stochastic white-noise perturbations of the memory, e.g.

$$
d x(t)=\left\{\int_{[-r, 0]} x(t+s) d W(s)\right\} d W(t), \quad t>0
$$

where $W$ is the standard one-dimensional Wiener process. It is interesting to note that the above sfde is not covered by classical results in sde's (cf. Protter [Pr], Metivier and Pellaumail [MP], Doleans-Dade [Do]).

At the end of the chapter we discuss mean-Lipschitz, smooth and/or sublinear dependence of the trajectory random field on the initial condition.

\section{Examples and motivation.}

Throughout this section, denote by $W: \mathbf{R}^{+} \times \Omega \rightarrow \mathbf{R}$ standard one-dimensional Wiener process defined on the canonical filtered Wiener space $\left(\Omega, \mathcal{F},\left(\mathcal{F}_{t}\right)_{t \in \mathbf{R}^{+}}, P\right)$, where $\Omega:=C\left(\mathbf{R}^{+}, \mathbf{R}\right), \mathcal{F}:=$ Borel $\Omega, \mathcal{F}_{t}:=\sigma\left\{\rho_{u}: u \leq t\right\}, \rho_{u}: \Omega \rightarrow \mathbf{R}, u \in \mathbf{R}^{+}$, are evaluation maps $\omega \mapsto \omega(u)$, and $P$ is Wiener measure on $\Omega$. 
Example 1. (Noisy Feedback Loops)

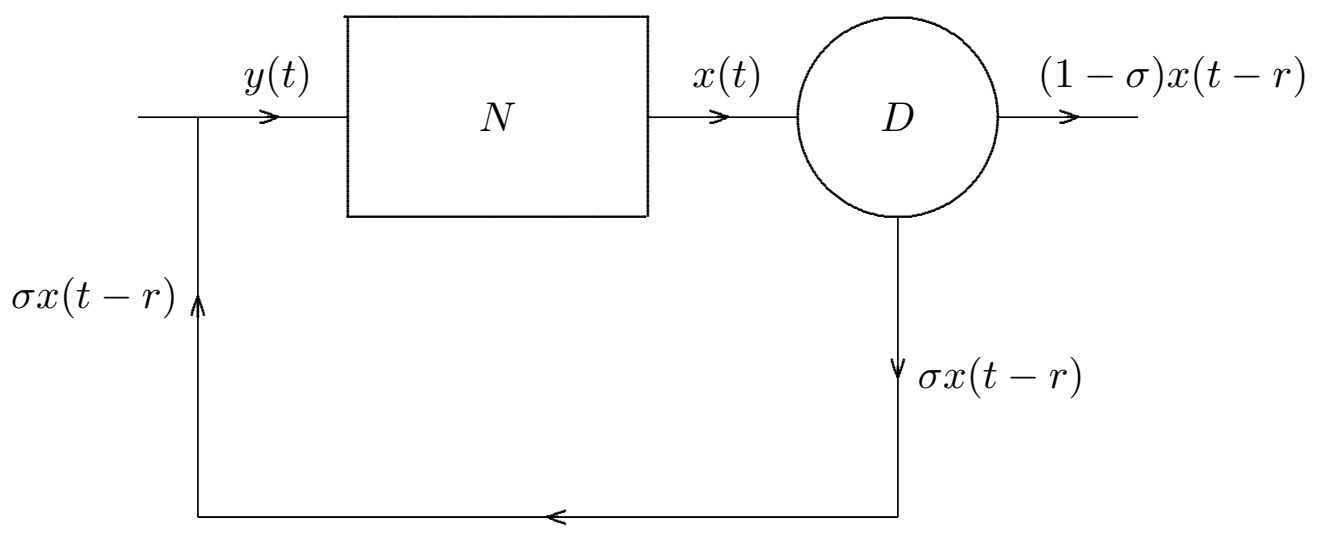

Consider the above noisy feedback loop. In Box $N$, the input $y(t)$ and output $x(t)$ at time $t>0$ are related through the stochastic integral

$$
x(t)=x(0)+\int_{0}^{t} y(u) d Z(u)
$$

where $Z(u)$ is a real-valued semimartingale noise. Unit $D$ delays the signal $x(t)$ by $r(>0)$ units of time. A proportion $\sigma(0 \leq \sigma \leq 1)$ of the signal is transmitted through the link $D$ and the rest $(1-\sigma)$ is used for other purposes. Therefore $y(t)=\sigma x(t-r)$. Take $\dot{Z}(u)$ to be white noise $\dot{W}(u)$. Substituting in (1), gives the Itô integral equation

$$
x(t)=x(0)+\sigma \int_{0}^{t} x(u-r) d W(u)
$$

or the stochastic differential delay equation (sdde):

$$
d x(t)=\sigma x(t-r) d W(t), \quad t>0 .
$$

In the nondelay case, $r=0$ and (I) becomes a linear stochastic ode with the closed-form solution

$$
x(t)=x(0) e^{\sigma W(t)-\left(\sigma^{2} t\right) / 2}, \quad t \geq 0 .
$$

Suppose the delay $r$ is positive. To solve (I), we need an initial process $\theta(t),-r \leq t \leq 0$, viz.

$$
x(t)=\theta(t) \quad \text { a.s., } \quad-r \leq t \leq 0 .
$$


We solve (I) by successive Itô integrations over steps of length $r$. This gives

$$
\begin{aligned}
& x(t)=\theta(0)+\sigma \int_{0}^{t} \theta(u-r) d W(u), \quad 0 \leq t \leq r, \\
& x(t)=x(r)+\sigma \int_{r}^{t}\left[\theta(0)+\sigma \int_{0}^{(v-r)} \theta(u-r) d W(u)\right] d W(v), r<t \leq 2 r, \\
& \quad 2 r<t \leq 3 r,
\end{aligned}
$$

No closed form solution is known (even in the deterministic case).

\section{Curious Fact!}

In the sdde (I), the Itô differential $d W$ may be replaced by the Stratonovich differential odW without changing the solution $x$. Let $x$ be the solution of (I) under an Itô differential $d W$. Then using finite partitions $\left\{u_{k}\right\}$ of the interval $[0, t]$, we have

$$
\int_{0}^{t} x(u-r) \circ d W(t)=\lim \sum_{k} \frac{1}{2}\left[x\left(u_{k}-r\right)+x\left(u_{k+1}-r\right)\right]\left[W\left(u_{k+1}\right)-W\left(u_{k}\right)\right]
$$

where the limit in probability is taken as the mesh of the partition $\left\{u_{k}\right\}$ goes to zero. Now compare the Stratonovich and Itô integrals using the corresponding partial sums. Thus

$$
\begin{aligned}
\lim E( & \sum_{k} \frac{1}{2}\left[x\left(u_{k}-r\right)+x\left(u_{k+1}-r\right)\right]\left[W\left(u_{k+1}\right)-W\left(u_{k}\right)\right] \\
& \left.-\sum_{k}\left[x\left(u_{k}-r\right)\right]\left[W\left(u_{k+1}\right)-W\left(u_{k}\right)\right]\right)^{2} \\
& =\lim E\left(\sum_{k} \frac{1}{2}\left[x\left(u_{k+1}-r\right)-x\left(u_{k}-r\right)\right]\left[W\left(u_{k+1}\right)-W\left(u_{k}\right)\right]\right)^{2} \\
& =\lim \sum_{k} \frac{1}{4} E\left[x\left(u_{k+1}-r\right)-x\left(u_{k}-r\right)\right]^{2} E\left[W\left(u_{k+1}\right)-W\left(u_{k}\right)\right]^{2} \\
& =\lim \sum_{k} \frac{1}{4} E\left[x\left(u_{k+1}-r\right)-x\left(u_{k}-r\right)\right]^{2}\left(u_{k+1}-u_{k}\right) \\
& =0
\end{aligned}
$$

because $W$ has independent increments, $x$ is adapted to the Brownian filtration, $u \mapsto$ $x(u) \in L^{2}(\Omega, \mathbf{R})$ is continuous, and the delay $r$ is positive. In fact the above computation shows that the quadratic variation $\langle x(\cdot-r, W\rangle(t)=0$ for all $t>0$, and

$$
\begin{aligned}
\int_{0}^{t} x(u-r) \circ d W(u) & =\int_{0}^{t} x(u-r) d W(u)+\frac{1}{2}<x(\cdot-r, W>(t) \\
& =\int_{0}^{t} x(u-r) d W(u)
\end{aligned}
$$


almost surely for all $t>0$.

\section{Remark.}

When $r>0$, the solution process $\{x(t): t \geq-r\}$ of $(\mathrm{I})$ is an $\left(\mathcal{F}_{t}\right)_{t \geq 0}$-martingale but is non-Markov.

\section{Example 2. (Simple Population Growth)}

Consider a large population $x(t)$ at time $t$ evolving with a constant birth rate $\beta>0$ and a constant death rate $\alpha$ per capita. Assume immediate removal of the dead from the population. Let the fixed non-random number $r>0$ denote the development period of each individual (e.g. $r=9$ months!). Assume there is migration whose overall rate is distributed like white noise $\sigma \dot{W}$. The change in population $\Delta x(t)$ over a small time interval $(t, t+\Delta t)$ is

$$
\Delta x(t)=-\alpha x(t) \Delta t+\beta x(t-r) \Delta t+\sigma \dot{W} \Delta t
$$

Letting $\Delta t \rightarrow 0$ and using Itô stochastic differentials, we obtain the sdde

$$
d x(t)=\{-\alpha x(t)+\beta x(t-r)\} d t+\sigma d W(t), \quad t>0 .
$$

We may associate with the above affine sdde the initial condition $(v, \eta) \in M_{2}:=\mathbf{R} \times$ $L^{2}([-r, 0], \mathbf{R})$

$$
x(0)=v, \quad x(s)=\eta(s), \quad-r \leq s<0 .
$$

The state space $M_{2}$ is the Delfour-Mitter Hilbert space consisting of all pairs $(v, \eta), v \in \mathbf{R}$, $\eta \in L^{2}([-r, 0], \mathbf{R})$ and furnished with the norm

$$
\|(v, \eta)\|_{M_{2}}:=\left(|v|^{2}+\int_{-r}^{0}|\eta(s)|^{2} d s\right)^{1 / 2} .
$$

\section{Example 3. (Logistic Population Growth)}

Consider a single population $x(t)$ at time $t$ evolving logistically with development (incubation) period $r>0$. Suppose there is migration on a molecular level which contributes $\gamma \dot{W}(t)$ to the growth rate per capita at time $t$. The evolution of the population is governed by the non-linear logistic sdde

$$
\dot{x}(t)=[\alpha-\beta x(t-r)] x(t)+\gamma x(t) \dot{W}(t), \quad t>0,
$$


i.e.

$$
d x(t)=[\alpha-\beta x(t-r)] x(t) d t+\gamma x(t) d W(t), \quad t>0 .
$$

with initial condition

$$
x(t)=\theta(t), \quad-r \leq t \leq 0,
$$

where $\eta:[-r, 0] \rightarrow \mathbf{R}$ is a continuous function.

For a positive delay $r$, the sdde (III) can be solved implicitly using forward steps of length $r$, i.e. for $0 \leq t \leq r, x(t)$ satisfies the linear stochastic ode (sode) (without delay):

$$
d x(t)=[\alpha-\beta \theta(t-r)] x(t) d t+\gamma x(t) d W(t) \quad 0<t \leq r .
$$

Note that $x(t)$ is an $\left(\mathcal{F}_{t}\right)_{t \geq 0}$-semimartingale and is non-Markov. This model was studied by Scheutzow $([\mathrm{S}])$.

Example 4. (Heat bath)

A model for "physical Brownian motion" was proposed by R. Kubo in $1966([\mathrm{~K}])$. A molecule of mass $m$ moves under random gas forces with position $\xi(t) \in \mathbf{R}^{3}$ and velocity $v(t) \in \mathbf{R}^{3}$ at time $t$; cf. classical work by Einstein and Ornstein and Uhlenbeck. Kubo proposed the following modification of the Ornstein-Uhlenbeck process

$$
\left.\begin{array}{rl}
d \xi(t) & =v(t) d t \\
m d v(t) & =-m\left[\int_{t_{0}}^{t} \beta\left(t-t^{\prime}\right) v\left(t^{\prime}\right) d t^{\prime}\right] d t+\gamma(\xi(t), v(t)) d W(t), t>t_{0} .
\end{array}\right\}
$$

In the above sfde, $\beta: \mathbf{R} \rightarrow \mathbf{R}^{+}$is a deterministic viscosity coefficient with compact support; $\gamma$ is a function $\mathbf{R}^{3} \times \mathbf{R}^{3} \rightarrow \mathbf{R}$ representing the random gas forces on the molecule; $W$ is 3-dimensional Brownian motion. This model is discussed in ([M1], pp. 223-226). See also Chapter VI, Section 3 of this article.

\section{Further Examples.}

We list here some further examples of sfde's.

First, consider the sdde with Poisson noise:

$$
\left.\begin{array}{rl}
d x(t) & =x((t-r)-) d N(t) \quad t>0 \\
x_{0} & =\eta \in D([-r, 0], \mathbf{R}) .
\end{array}\right\}
$$

In the above sdde, $N$ is a Poisson process with i.i.d. interarrival times $([\mathrm{S}]) ; D([-r, 0], \mathbf{R})$ is the space of all cadlag paths $[-r, 0] \rightarrow \mathbf{R}$, given the supremum norm. Large-time asymptotics of (V) are given in Chapter V, Section 3. 
The figure below represents a simple model of dye circulation in the blood stream (or pollution in a river) (cf. [BW], [LT]).

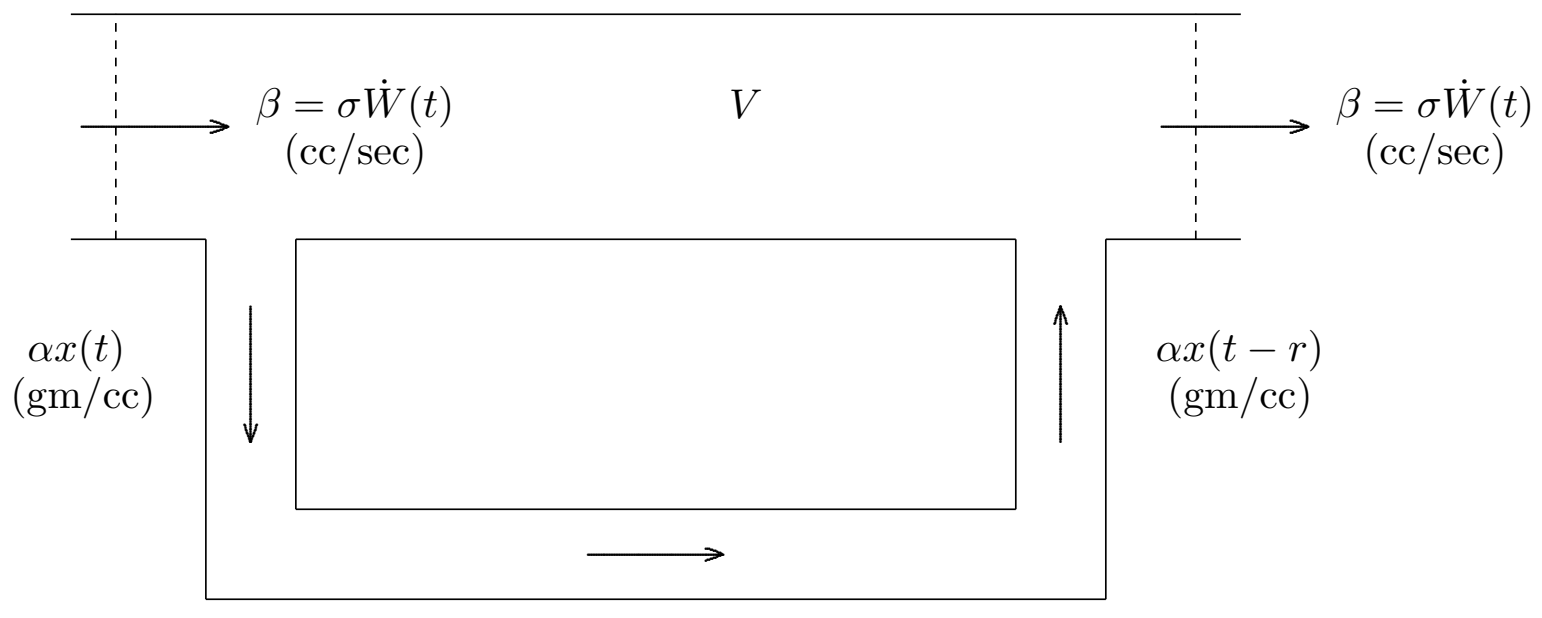

The main blood vessel has dye with concentration $x(t)(\mathrm{gm} / \mathrm{cc})$ at time $t$. A fixed proportion of blood in the main vessel is pumped into the side vessel(s). The blood takes $r>0$ seconds to traverse the side tube (vessel). Assume that the flow rate $(\mathrm{cc} / \mathrm{sec})$ in the main blood vessel is Gaussian with constant mean and variance $\sigma$. By writing an equation for the rate of dye transfer through a fixed part $V$ of the main vessel, it is easy to see that the following sdde holds:

$$
\left.\begin{array}{rl}
d x(t) & =\{\nu x(t)+\mu x(t-r)\} d t+\sigma x(t) d W(t), \quad t>0 \\
\left(x(0), x_{0}\right) & =(v, \eta) \in M_{2}=\mathbf{R} \times L^{2}([-r, 0], \mathbf{R}),
\end{array}\right\}
$$

where $\nu$ and $\mu$ are real constants. The above model will be analyzed in Chapter V (Theorem V.5). See also the survey article ([M4]) and ([MS2]).

The following sfde has discrete lag in the drift but a distributed delay in the diffusion term:

$$
\left.\begin{array}{rl}
d x(t) & =\{\nu x(t)+\mu x(t-r))\} d t+\left\{\int_{-r}^{0} x(t+s) \sigma(s) d s\right\} d W(t), t>0 \\
\left(x(0), x_{0}\right) & =(v, \eta) \in M_{2}=\mathbf{R} \times L^{2}([-r, 0], \mathbf{R}) .
\end{array}\right\}
$$

$([\mathrm{M} 4],[\mathrm{MS} 2])$. 
In Chapter IV, we will study the following system of linear $d$-dimensional sfde's driven by $m$-dimensional Brownian motion $W:=\left(W_{1}, \cdots, W_{m}\right)$ :

$$
\left.\begin{array}{c}
d x(t)=\left\{\int_{-r}^{0} h\left(s, x\left(t-d_{1}\right), \cdots, x\left(t-d_{N}\right), x(t), x(t+s)\right) d s\right\} d t \\
+\sum_{i=1}^{m} g_{i} x(t) d W_{i}(t), \quad t>0 \\
\left(x(0), x_{0}\right)=(v, \eta) \in M_{2}:=\mathbf{R}^{d} \times L^{2}\left([-r, 0], \mathbf{R}^{d}\right)
\end{array}\right\}
$$

In $\left(\mathrm{VIII}^{\prime}\right), h(s, \cdots):\left(\mathbf{R}^{d}\right)^{N+2} \rightarrow \mathbf{R}^{d}$ is a linear map for each $s \in[-r, 0]$, and each $g_{i}$, $1 \leq i \leq d$, is a $d \times d$-matrix ([M3]).

The following is a more general class of linear systems of sfde's:

$$
\left.\begin{array}{rl}
d x(t)= & \left\{\int_{[-r, 0]} \nu(t)(d s) x(t+s)\right\} d t \\
& +d N(t) \int_{-r}^{0} K(t)(s) x(t+s) d s+d L(t) x(t-), \quad t>0 \\
\left(x(0), x_{0}\right)= & (v, \eta) \in M_{2}=\mathbf{R}^{d} \times L^{2}\left([-r, 0], \mathbf{R}^{d}\right)
\end{array}\right\}
$$

In the above equation, $\nu$ takes values in the $\mathbf{R}^{d \times d}$-valued measures, $K(t)(s)$ is a stationary (in $t$ ) $\mathbf{R}^{d \times d}$-valued process and $L$ is an $\mathbf{R}^{d \times d}$-valued semimartingale with stationary ergodic increments. The ergodic theory of equation (IX) will be treated in Chapter IV. See also $([\mathrm{MS} 1])$.

Multidimensional affine systems driven by a (helix) noise $Q$ will be discussed briefly in Chapter (VI) ([MS3]):

$$
\left.\begin{array}{rl}
d x(t) & =\left\{\int_{[-r, 0]} \nu(t)(d s) x(t+s)\right\} d t+d Q(t), \quad t>0 \\
\left.0), x_{0}\right) & =(v, \eta) \in M_{2}:=\mathbf{R}^{d} \times L^{2}\left([-r, 0], \mathbf{R}^{d}\right)
\end{array}\right\}
$$

In the following one-dimensional sfde, the memory is driven by white noise:

$$
\left.\begin{array}{l}
d x(t)=\left\{\int_{[-r, 0]} x(t+s) d W(s)\right\} d W(t), \quad t>0 \\
x(0)=v \in \mathbf{R}, \quad x(s)=\eta(s), \quad-r<s<0, \quad r \geq 0
\end{array}\right\}
$$




\section{General formulation. Existence and uniqueness.}

In this chapter and throughout the article, the symbol $|\cdot|$ denotes the Euclidean norm on $\mathbf{R}^{d}$.

At each $t \geq 0$, slice each solution path $x:[-r, \infty) \rightarrow \mathbf{R}^{d}$ over the interval $[t-r, t]$ and define the segment $x_{t}:[-r, 0] \rightarrow \mathbf{R}^{d}$ by

$$
x_{t}(s):=x(t+s) \quad \text { a.s., } t \geq 0, s \in J:=[-r, 0] .
$$

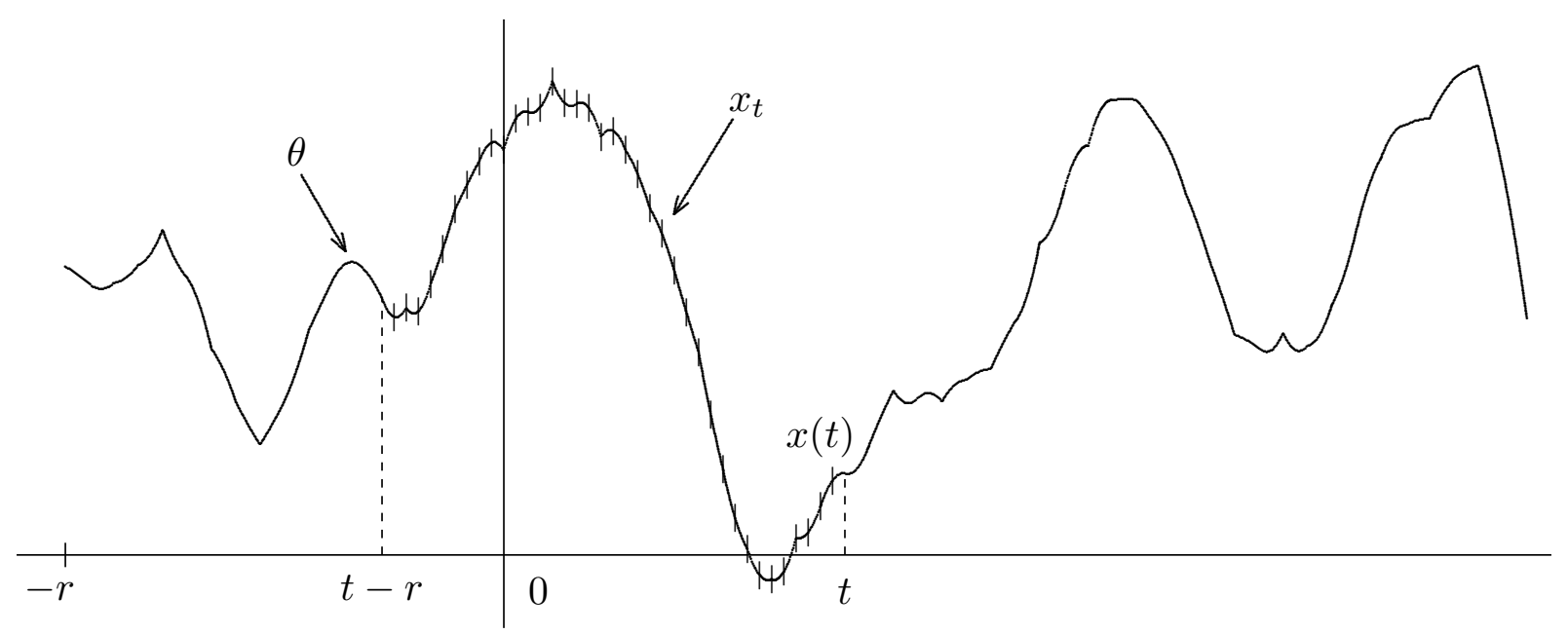

Therefore the sdde's (I), (II), (III) and (XI) become

$$
\left.\begin{array}{c}
d x(t)=\sigma x_{t}(-r) d W(t), \quad t>0 \\
\left.x_{0}=\theta \in C([-r, 0], \mathbf{R}) \quad\right\} \\
d x(t)=\left\{-\alpha x(t)+\beta x_{t}(-r)\right\} d t+\sigma d W(t), \quad t>0 \\
\left.(0), x_{0}\right)=(v, \eta) \in \mathbf{R} \times L^{2}([-r, 0], \mathbf{R}) \\
\left.d x(t)=\left[\alpha-\beta x_{t}(-r)\right] x_{t}(0) d t+\gamma x_{t}(0) d W(t)\right\} \\
x_{0}=\theta \in C([-r, 0], \mathbf{R}) \\
d x(t)=\left\{\int_{[-r, 0]} x_{t}(s) d W(s)\right\} d W(t) \quad t>0 \\
\left(x(0), x_{0}\right)=(v, \eta) \in \mathbf{R} \times L^{2}([-r, 0], \mathbf{R}), \quad r \geq 0
\end{array}\right\}
$$


The right-hand sides of equations (I), (II), (III), (XI) may be viewed as functionals of $x_{t}$ (and $x(t)$ ). Therefore we can imbed these equations in the following general class of stochastic functional differential equations (sfde's)

$$
\left.\begin{array}{rl}
d x(t) & =h\left(t, x_{t}\right) d t+g\left(t, x_{t}\right) d W(t), \quad t>0 \\
x_{0} & =\theta
\end{array}\right\}
$$

on a filtered probability space $\left(\Omega, \mathcal{F},\left(\mathcal{F}_{t}\right)_{t \geq 0}, P\right)$ satisfying the usual conditions; viz. the filtration $\left(\mathcal{F}_{t}\right)_{t \geq 0}$ is right-continuous, and each $\mathcal{F}_{t}, t \geq 0$, contains all $P$-null sets in $\mathcal{F}$. Denote by $C:=C\left([-r, 0], \mathbf{R}^{d}\right)$ the Banach space of all continuous paths $[-r, 0] \rightarrow \mathbf{R}^{d}$ given the supremum norm

$$
\|\eta\|_{C}:=\sup _{s \in[-r, 0]}|\eta(s)|, \quad \eta \in C .
$$

In the sfde (XII), $W(t)$ represents $m$-dimensional Brownian motion and $L^{2}(\Omega, C)$ is the Banach space of all (equivalence classes of) $(\mathcal{F}$, Borel $C$ )-measurable maps $\Omega \rightarrow C$ which are $L^{2}$ in the Bochner sense. Give $L^{2}(\Omega, C)$ the Banach norm

$$
\|\theta\|_{L^{2}(\Omega, C)}:=\left[\int_{\Omega}\|\theta(\omega)\|_{C}^{2} d P(\omega)\right]^{1 / 2} .
$$

The sfde (XII) has a drift coefficient function $h:[0, T] \times L^{2}(\Omega, C) \rightarrow L^{2}\left(\Omega, \mathbf{R}^{d}\right)$ and a diffusion coefficient function $g:[0, T] \times L^{2}(\Omega, C) \rightarrow L^{2}\left(\Omega, \mathbf{R}^{d \times m}\right)$ satisfying Hypotheses $\left(E_{1}\right)$ below. The initial path is an $\mathcal{F}_{0}$-measurable process $\theta \in L^{2}\left(\Omega, C ; \mathcal{F}_{0}\right)$.

A solution of (XII) is a measurable, sample-continuous process $x:[-r, T] \times \Omega \rightarrow \mathbf{R}^{d}$ such that $x \mid[0, T]$ is $\left(\mathcal{F}_{t}\right)_{0 \leq t \leq T}$-adapted, $x(s)$ is $\mathcal{F}_{0}$-measurable for all $s \in[-r, 0]$, and $x$ satisfies(XII) almost surely.

Note that the path-valued trajectory $[0, T] \ni t \mapsto x_{t} \in C\left([-r, 0], \mathbf{R}^{d}\right)$ is $\left(\mathcal{F}_{t}\right)_{0 \leq t \leq T^{-}}$ adapted. This is because Borel $C$ is generated by all evaluations $C \ni \eta \mapsto \eta(s) \in \mathbf{R}^{d}, s \in$ $J$.

\section{Hypotheses $\left(E_{1}\right)$.}

(i) The coefficient functionals $h$ and $g$ are jointly continuous and uniformly Lipschitz in the second variable with respect to the first, viz.

$$
\left\|h\left(t, \psi_{1}\right)-h\left(t, \psi_{2}\right)\right\|_{L^{2}\left(\Omega, \mathbf{R}^{d}\right)}+\left\|g\left(t, \psi_{1}\right)-g\left(t, \psi_{2}\right)\right\|_{L^{2}\left(\Omega, \mathbf{R}^{d \times m}\right)} \leq L\left\|\psi_{1}-\psi_{2}\right\|_{L^{2}(\Omega, C)}
$$

for all $t \in[0, T]$ and $\psi_{1}, \psi_{2} \in L^{2}(\Omega, C)$. The Lipschitz constant $L$ is independent of $t \in[0, T]$.

(ii) For each $\left(\mathcal{F}_{t}\right)_{0 \leq t \leq T}$-adapted process $y:[0, T] \rightarrow L^{2}(\Omega, C)$, the processes $h(\cdot, y(\cdot))$ and $g(\cdot, y(\cdot))$ are also $\left(\mathcal{F}_{t}\right)_{0 \leq t \leq T^{-}}$adapted. 
Theorem I.1. (Existence and Uniqueness)([M1])

Suppose $h$ and $g$ satisfy Hypotheses $\left(E_{1}\right)$. Let $\theta \in L^{2}\left(\Omega, C ; \mathcal{F}_{0}\right)$.

Then the sfde (XII) has a unique solution ${ }^{\theta} x:[-r, \infty) \times \Omega \rightarrow \mathbf{R}^{d}$ starting off at $\theta \in$ $L^{2}\left(\Omega, C ; \mathcal{F}_{0}\right)$ with $[0, T] \ni t \longmapsto{ }^{\theta} x_{t} \in C$ sample-continuous, and ${ }^{\theta} x \in L^{2}\left(\Omega, C\left([-r, T], \mathbf{R}^{d}\right)\right)$ for all $T>0$. For a given $\theta$, uniqueness holds up to equivalence among all $\left(\mathcal{F}_{t}\right)_{0 \leq t \leq T^{-}}$ adapted processes in $L^{2}\left(\Omega, C\left([-r, T], \mathbf{R}^{d}\right)\right)$.

The proof of the above theorem uses a classical successive approximation technique. The essence of the argument will be outlined in the proof of Theorem I.2 below. See also ([M1], Theorem 2.1, pp. 36-39.)

Theorem I.1 covers equations (I), (II), (IV), (VI), (VII), (VIII), (XI) and a large class of sfde's driven by white noise. Note that (XI) does not satisfy the hypotheses underlying the classical results of Doleans-Dade [Do], Metivier and Pellaumail [MP], Protter [P1], Lipster and Shiryayev [LS], and Metivier [Met]. This is because the coefficient functional

$$
\eta \mapsto \int_{-r}^{0} \eta(s) d W(s)
$$

on the right-hand side of (XI) does not admit almost surely Lipschitz (or even linear) versions $C \rightarrow \mathbf{R}$ ! This will be shown later (Chapter V, Section 1 ).

When the coefficients $h$ and $g$ in (XII) factor through (deterministic) functionals

$$
H:[0, T] \times C \rightarrow \mathbf{R}^{d}, \quad G:[0, T] \times C \rightarrow \mathbf{R}^{d \times m},
$$

we can impose the following local Lipschitz and global linear growth conditions on the sfde

$$
\left.\begin{array}{rl}
d x(t) & =H\left(t, x_{t}\right) d t+G\left(t, x_{t}\right) d W(t), \quad t>0 \\
x_{0} & =\theta
\end{array}\right\}
$$

where $W$ is $m$-dimensional Brownian motion:

Hypotheses $\left(E_{2}\right)$.

(i) Suppose that $H$ and $G$ are Lipschitz on bounded sets in $C$ uniformly in the second variable; viz. for each integer $n \geq 1$, there exists a constant $L_{n}>0$ (independent of $t \in[0, T])$ such that

$$
\left|H\left(t, \eta_{1}\right)-H\left(t, \eta_{2}\right)\right|+\left\|G\left(t, \eta_{1}\right)-G\left(t, \eta_{2}\right)\right\| \leq L_{n}\left\|\eta_{1}-\eta_{2}\right\|_{C}
$$

for all $t \in[0, T]$ and $\eta_{1}, \eta_{2} \in C$ with $\left\|\eta_{1}\right\|_{C} \leq n,\left\|\eta_{2}\right\|_{C} \leq n$. 
(ii) There is a constant $K>0$ such that

$$
|H(t, \eta)|+\|G(t, \eta)\| \leq K\left(1+\|\eta\|_{C}\right)
$$

for all $t \in[0, T]$ and $\eta \in C$.

Note that the adaptability condition is not needed (explicitly) in (XIII) because $H$ and $G$ are deterministic, and because the sample-continuity and adaptability of $x$ imply that the segment $[0, T] \ni t \mapsto x_{t} \in C$ is also adapted.

Assuming that $\beta$ has compact support in $\mathbf{R}^{+}$, the heat-bath model (IV) may easily be formulated as a sfde of the form (XIII).

Theorem I.2. (Existence and Uniqueness)([M1])

Suppose $H$ and $G$ satisfy Hypotheses $\left(E_{2}\right)$ and let $\theta \in L^{2}\left(\Omega, C ; \mathcal{F}_{0}\right)$.

Then the sfde (XIII) has a unique $\left(\mathcal{F}_{t}\right)_{0 \leq t \leq T}$-adapted solution ${ }^{\theta} x:[-r, T] \times \Omega \rightarrow \mathbf{R}^{d}$ starting off at $\theta \in L^{2}\left(\Omega, C ; \mathcal{F}_{0}\right)$ with $[0, T] \ni t \longmapsto{ }^{\theta} x_{t} \in C$ sample-continuous, and ${ }^{\theta} x \in L^{2}\left(\Omega, C\left([-r, T], \mathbf{R}^{d}\right)\right)$ for all $T>0$. For a given $\theta$, uniqueness holds up to equivalence among all $\left(\mathcal{F}_{t}\right)_{0 \leq t \leq T}$-adapted processes in $L^{2}\left(\Omega, C\left([-r, T], \mathbf{R}^{d}\right)\right)$.

Furthermore, if $\theta \in L^{2 k}\left(\Omega, C ; \mathcal{F}_{0}\right)$ for some positive integer $k$, then ${ }^{\theta} x_{t} \in L^{2 k}\left(\Omega, C ; \mathcal{F}_{t}\right)$ and

$$
E\left\|^{\theta} x_{t}\right\|_{C}^{2 k} \leq C_{k}\left[1+\|\theta\|_{L^{2 k}(\Omega, C)}^{2 k}\right]
$$

for all $t \in[0, T]$ and some positive constant $C_{k}$.

Outline of Proofs of Theorems I.1, I.2.

We will outline the proofs of Theorems I.1 and I.2. The proofs are based on the classical successive approximation scheme; cf. ([M1], pp. 150-152, [GS] or [Fr]).

The main steps of the proof are as follows:

(1) Truncate the coefficients of (XIII) outside any open ball of radius $N$ in $C$, using globally Lipschitz partitions of unity. This, together with Step 3 below, reduces the problem of existence of a solution to the case with globally Lipschitz coefficients.

(2) Assuming globally Lipschitz coefficients, use successive approximations to obtain a unique pathwise solution of the sfde (XII). Details of this argument are given below.

(3) Under a global Lipschitz hypothesis, it is possible to show that for sfde's of type (XIII), if the coefficients agree on an open set $U$ in $C$, then the trajectories starting 
from an initial path in $U$ must leave $U$ at the same time and agree until they leave $U$. Now truncate each coefficient in (XIII) outside an open ball of radius $N$ and center 0 in $C$. Using the above local uniqueness result, one can "patch up" solutions of the truncated sfde's as $N$ increases to infinity ([M1], pp. 150-151).

We shall only give the argument for (2). Suppose for simplicity that $h \equiv 0$ in (XII) and $g$ satisfies the global Lipschitz condition $\left(E_{1}\right)(i)$.

Let $J:=[-r, 0]$. Denote by $L_{A}^{2}\left(\Omega, C\left([-r, a], \mathbf{R}^{d}\right)\right)$ the space of all processes $x \in$ $L^{2}\left(\Omega, C\left([-r, a], \mathbf{R}^{d}\right)\right)$ such that $x(s, \cdot):=x(\cdot)(s)$ is $\mathcal{F}_{0}$-measurable for all $s \in J$ and $x(t, \cdot)$ is $\mathcal{F}_{t^{-}}$-measurable for all $t \in[0, a]$. Clearly $L_{A}^{2}\left(\Omega, C\left([-r, a], \mathbf{R}^{d}\right)\right)$ is a closed linear subspace of $L^{2}\left(\Omega, C\left([-r, a], \mathbf{R}^{d}\right)\right)$. We will look for solutions of (XII) by successive approximation in $L_{A}^{2}\left(\Omega, C\left([-r, a], \mathbf{R}^{d}\right)\right)$.

Suppose that $\theta \in L^{2}\left(\Omega, C\left(J, \mathbf{R}^{d}\right)\right)$ is $\mathcal{F}_{0}$-measurable. Note that this is equivalent to saying that $\theta(s, \cdot):=\theta(\cdot)(s)$ is $\mathcal{F}_{0}$-measurable for all $s \in J$, because $\theta$ has a.a. sample paths continuous.

We prove by induction that there is a sequence of processes ${ }^{k} x:[-r, a] \times \Omega \rightarrow \mathbf{R}^{d}$, $k=1,2, \cdots$ having the

Properties $P(k)$ :

(i) ${ }^{k} x \in L_{A}^{2}\left(\Omega, C\left([-r, a], \mathbf{R}^{d}\right)\right)$.

(ii) For each $t \in[0, a],{ }^{k} x_{t} \in L^{2}\left(\Omega, C\left(J, \mathbf{R}^{d}\right) ; \mathcal{F}_{t}\right)$.

$$
\left.\begin{array}{rl}
\left\|^{k+1} x-{ }^{k} x\right\|_{L^{2}(\Omega, C)} & \leq\left(M L^{2}\right)^{k-1} \frac{a^{k-1}}{(k-1) !}\left\|^{2} x-{ }^{1} x\right\|_{L^{2}(\Omega, C)} \\
\left\|^{k+1} x_{t}-{ }^{k} x_{t}\right\|_{L^{2}(\Omega, C)} & \leq\left(M L^{2}\right)^{k-1} \frac{t^{k-1}}{(k-1) !}\left\|^{2} x-{ }^{1} x\right\|_{L^{2}(\Omega, C)}
\end{array}\right\}
$$

where $M$ is a "martingale" constant and $L$ is the Lipschitz constant of $g$.

Define ${ }^{1} x:[-r, a] \times \Omega \rightarrow \mathbf{R}^{d}$ by

$$
{ }^{1} x(t, \omega)= \begin{cases}\theta(0, \omega), & t \in[0, a] \\ \theta(t, \omega), & t \in J\end{cases}
$$

a.s., and

$$
{ }^{k+1} x(t, \omega)=\left\{\begin{array}{l}
\theta(0, \omega)+(\omega) \int_{0}^{t} g\left(u,{ }^{k} x_{u}\right) d W(\cdot)(u), \quad t \in[0, a] \\
\theta(t, \omega), \quad t \in J
\end{array}\right.
$$

a.s.. 
Since $\theta \in L^{2}\left(\Omega, C\left(J, \mathbf{R}^{d}\right) ; \mathcal{F}_{0}\right)$, then ${ }^{1} x \in L_{A}^{2}\left(\Omega, C\left([-r, a], \mathbf{R}^{d}\right)\right)$. Therefore ${ }^{1} x_{t} \in$ $L^{2}\left(\Omega, C\left(J, \mathbf{R}^{d}\right) ; \mathcal{F}_{t}\right)$. Note that property $P(1)$ (iii) holds trivially.

Now suppose $P(k)$ is satisfied for some $k>1$. It is easy to see that the "slicing map" $[0, a] \times L^{2}\left(\Omega, C\left([-r, a], \mathbf{R}^{d}\right)\right) \ni(u, x) \mapsto x_{u} \in L^{2}\left(\Omega, C\left(J, \mathbf{R}^{d}\right)\right)$ is continuous. Then by Hypothesis $\left(E_{1}\right)(\mathrm{i})$, (ii) and property $P(k)($ ii) it follows that the process

$$
[0, a] \ni u \longmapsto g\left(u,{ }^{k} x_{u}\right) \in L^{2}\left(\Omega, \mathbf{R}^{d \times m}\right)
$$

is continuous and adapted to $\left(\mathcal{F}_{t}\right)_{t \in[0, a]}$. Properties $P(k+1)(\mathrm{i})$ and $P(k+1)$ (ii) follow from the continuity and adaptability of the stochastic integral. Property $P(k+1)($ iii $)$ is easily checked by using Doob's inequality.

For each $k>1$, write

$$
{ }^{k} x={ }^{1} x+\sum_{i=1}^{k-1}\left({ }^{i+1} x-{ }^{i} x\right)
$$

Since $L_{A}^{2}\left(\Omega, C\left([-r, a], \mathbf{R}^{d}\right)\right)$ is closed in $L^{2}\left(\Omega, C\left([-r, a], \mathbf{R}^{d}\right)\right)$, the series

$$
\sum_{i=1}^{\infty}\left({ }^{i+1} x-{ }^{i} x\right)
$$

converges in $L_{A}^{2}\left(\Omega, C\left([-r, a], \mathbf{R}^{d}\right)\right)$ because of (1) and the convergence of

$$
\sum_{i=1}^{\infty}\left[\left(M L^{2}\right)^{i-1} \frac{a^{i-1}}{(i-1) !}\right]^{1 / 2}
$$

Hence $\left\{{ }^{k} x\right\}_{k=1}^{\infty}$ converges to some $x \in L_{A}^{2}\left(\Omega, C\left([-r, a], \mathbf{R}^{d}\right)\right)$.

Clearly $x \mid J=\theta$ and is $\mathcal{F}_{0}$-measurable, so applying Doob's inequality to the Itô integral of the difference

$$
u \longmapsto g\left(u,{ }^{k} x_{u}\right)-g\left(u, x_{u}\right),
$$

gives

$$
E\left(\sup _{t \in[0, a]}\left|\int_{0}^{t} g\left(u,{ }^{k} x_{u}\right) d W(\cdot)(u)-\int_{0}^{t} g\left(u, x_{u}\right) d W(\cdot)(u)\right|^{2}\right) \leq M L^{2} a\left\|^{k} x-x\right\|_{L^{2}(\Omega, C)}^{2}
$$

for all $k \geq 1$. Thus, viewing the right-hand side of (2) as a process in $L^{2}\left(\Omega, C\left([-r, a], \mathbf{R}^{d}\right)\right)$ and letting $k \rightarrow \infty$, it follows from the above inequality that $x$ must satisfy the sfde (XII) a.s. for all $t \in[-r, a]$. 
For uniqueness, let $\tilde{x} \in L_{A}^{2}\left(\Omega,\left([-r, a], \mathbf{R}^{d}\right)\right)$ be also a solution of (XII) with initial process $\theta$. Then by the Lipschitz condition, we get

$$
\left\|x_{t}-\tilde{x}_{t}\right\|_{L^{2}(\Omega, C)}^{2}<M L^{2} \int_{0}^{t}\left\|x_{u}-\tilde{x}_{u}\right\|_{L^{2}(\Omega, C)}^{2} d u
$$

for all $t \in[0, a]$. Therefore we must have $x_{t}-\tilde{x}_{t}=0$ for all $t \in[0, a]$. Hence $x=\tilde{x}$ in $L^{2}\left(\Omega, C\left([-r, a], \mathbf{R}^{d}\right)\right)$.

\section{Remarks and generalizations.}

(i) In Theorem I.2, it is possible to replace the process $(t, W(t))$ by a (square integrable) semimartingale $Z(t)$ satisfying appropriate conditions ([M1], Chapter II).

(ii) Results on the existence of solutions of sfde's driven by white noise were first obtained by Itô and Nisio in their pioneering work ([IN], 1968) and then later by Kushner ([Kus], 1976).

(iii) For extensions to sfde's with general infinite memory, see [IN]. The fading memory case was treated by Mizel and Trutzer ([MT], 1984), Marcus and Mizel ([MM], 1988).

(iv) With minor changes in the arguments, the state space $C$ in Theorems I.1, I.2 may be replaced by the Delfour-Mitter Hilbert space $M_{2}:=\mathbf{R}^{d} \times L^{2}\left([-r, 0], \mathbf{R}^{d}\right)$ with the Hilbert norm

$$
\|(v, \eta)\|_{M_{2}}=\left(|v|^{2}+\int_{-r}^{0}|\eta(s)|^{2} d s\right)^{1 / 2}
$$

for $(v, \eta) \in M_{2}([\mathrm{~A}], 1983)$.

(v) Under Lipschitz (and/or Fréchet smoothness) hypotheses on the coefficients $h$ and $g$ in (XII), it follows that the map

$$
L^{2}\left(\Omega, C ; \mathcal{F}_{0}\right) \ni \theta \mapsto{ }^{\theta} x_{t} \in L^{2}\left(\Omega, C ; \mathcal{F}_{t}\right)
$$

is Lipschitz (and/or Fréchet smooth) (resp.) for each fixed $t \in[0, a]$ ([M1], Theorems $3.1,3.2$, pp. 41-45). 


\section{Chapter II}

\section{Markov Behavior and the Generator}

Consider the sfde

$$
\left.\begin{array}{rl}
d x(t) & =H\left(t, x_{t}\right) d t+G\left(t, x_{t}\right) d W(t), \quad t>0 \\
x_{0} & =\eta \in C:=C\left([-r, 0], \mathbf{R}^{d}\right)
\end{array}\right\}
$$

with coefficients $H:[0, T] \times C \rightarrow \mathbf{R}^{d}, \quad G:[0, T] \times C \rightarrow \mathbf{R}^{d \times m}$, driven by $m$-dimensional Brownian motion $W$. Let $\left\{{ }^{\eta} x_{t}: t \geq 0, \eta \in C\right\}$ denote the trajectory field of (XIII).

It would be interesting to give satisfactory answers to the following questions regarding the sfde (XIII):

(i) Does the trajectory field $x_{t}$ of (XIII) give a diffusion in $C$ (or $\left.M_{2}\right)$ ?

(ii) How does the trajectory $x_{t}$ transform under smooth non-linear functionals $\phi: C \rightarrow$ $\mathbf{R}$ ?

(iii) What "diffusions" on $C$ (or $M_{2}$ ) correspond to sfde's on $\mathbf{R}^{d}$ ?

This chapter is an attempt to answer the first two questions. Question (iii) is still largely open.

We begin by outlining the main difficulties encountered in answering questions (i) and (ii) above.

\section{Difficulties.}

(i) Although the current state $x(t)$ in (XIII) is a semimartingale, the trajectory $x_{t}$ does not seem to possess any (semi)martingale properties when viewed as $C$-(or $\left.M_{2}\right)$-valued process; e.g. for Brownian motion $W(H \equiv 0, G \equiv 1)$, one has

$$
\left[E\left(W_{t} \mid \mathcal{F}_{t_{1}}\right)\right](s)=W\left(t_{1}\right)=W_{t_{1}}(0), \quad s \in[-r, 0]
$$

whenever $t_{1} \leq t-r$.

(ii) We will show that $x_{t}$ is a Markov process in $C$. However, the underlying Markov semigroup turns out to be not strongly continuous with respect the topology of uniform convergence on the space of all bounded continuous functions on $C$. Such lack of strong continuity leads to the use of weak limits in $C$ which tend to live outside $C$. 
(iii) Almost all tame functions will be shown to lie outside the domain of the (weak) generator of the Markov semigroup.

(iv) The absence of an Itô formula for the trajectory $x_{t}$ makes the computation of the weak infinitesimal generator especially hard. This difficulty is circumvented by appealing to functional-analytic methods.

\section{The Markov property.}

In this section we will show that the trajectory field $x_{t}$ corresponds to a $C$-valued Markov process. The following hypotheses will be needed.

\section{Hypotheses (M).}

(i) For each $t \geq 0, \mathcal{F}_{t}$ is the completion of the $\sigma$-algebra $\sigma\{W(u): 0 \leq u \leq t\}$.

(ii) $H$ and $G$ are jointly continuous and globally Lipschitz in the second variable uniformly with respect to the first:

$$
\left|H\left(t, \eta_{1}\right)-H\left(t, \eta_{2}\right)\right|+\left\|G\left(t, \eta_{1}\right)-G\left(t, \eta_{2}\right)\right\| \leq L\left\|\eta_{1}-\eta_{2}\right\|_{C}
$$

for all $t \in[0, T]$ and $\eta_{1}, \eta_{2} \in C$.

Consider the sfde

$$
{ }^{\theta} x^{t_{1}}(t)=\left\{\begin{array}{l}
\theta(0)+\int_{t_{1}}^{t} H\left(u,{ }^{\theta} x_{u}^{t_{1}}\right) d u+\int_{t_{1}}^{t} G\left(u,{ }^{\theta} x_{u}^{t_{1}}\right) d W(u), \quad t>t_{1}, \\
\theta\left(t-t_{1}\right), \quad t_{1}-r \leq t \leq t_{1} .
\end{array}\right.
$$

Let ${ }^{\theta} x^{t_{1}}$ be its solution, starting off at $\theta \in L^{2}\left(\Omega, C ; \mathcal{F}_{t_{1}}\right)$ at $t=t_{1}$. This gives a twoparameter family of mappings

$$
\begin{gathered}
T_{t_{2}}^{t_{1}}: L^{2}\left(\Omega, C ; \mathcal{F}_{t_{1}}\right) \rightarrow L^{2}\left(\Omega, C ; \mathcal{F}_{t_{2}}\right), t_{1} \leq t_{2} \\
T_{t_{2}}^{t_{1}}(\theta):={ }^{\theta} x_{t_{2}}^{t_{1}}, \quad \theta \in L^{2}\left(\Omega, C ; \mathcal{F}_{t_{1}}\right)
\end{gathered}
$$

The uniqueness of solutions to the above sfde gives the two-parameter semigroup property:

$$
T_{t_{2}}^{t_{1}} \circ T_{t_{1}}^{0}=T_{t_{2}}^{0}, \quad t_{1} \leq t_{2}
$$

([M1], Theorem II (2.2), p. 40). 
Theorem II.1. (Markov Property)([M1])

In (XIII), suppose Hypotheses (M) hold. Then the trajectory field $\left\{{ }^{\eta} x_{t}: t \geq 0, \eta \in\right.$ $C\}$ is a $C$-valued Feller process with transition probabilities

$$
p\left(t_{1}, \eta, t_{2}, B\right):=P\left({ }^{\eta} x_{t_{2}}^{t_{1}} \in B\right), \quad t_{1} \leq t_{2}, \quad B \in \text { Borel } C, \quad \eta \in C
$$

i.e.

$$
P\left(x_{t_{2}} \in B \mid \mathcal{F}_{t_{1}}\right)=p\left(t_{1}, x_{t_{1}}(\cdot), t_{2}, B\right)=P\left(x_{t_{2}} \in B \mid x_{t_{1}}\right) \quad \text { a.s. }
$$

Furthermore, if $H$ and $G$ do not depend on $t$, then the trajectory is time-homogeneous:

$$
p\left(t_{1}, \eta, t_{2}, \cdot\right)=p\left(0, \eta, t_{2}-t_{1}, \cdot\right), \quad 0 \leq t_{1} \leq t_{2}, \quad \eta \in C .
$$

Proof.

The first equality in (3) is equivalent to

$$
\int_{A} 1_{B}\left(T_{t_{2}}^{0}(\theta)(\omega)\right) d P(\omega)=\int_{A} \int_{\Omega} 1_{B}\left\{\left[T_{t_{2}}^{t_{1}}\left(T_{t_{1}}^{0}(\theta)\left(\omega^{\prime}\right)\right)\right](\omega)\right\} d P(\omega) d P\left(\omega^{\prime}\right)
$$

for all $A \in \mathcal{F}_{t_{1}}$ and all Borel subsets $B$ of $C$. The symbol $1_{B}$ denotes the indicator function of $B \subseteq C$. In order to prove the equality (5), observe first that it holds when $1_{B}$ is replaced by an arbitrary uniformly continuous and bounded function $\phi: C \rightarrow \mathbf{R}$; that is

$$
\int_{A} \phi\left(T_{t_{2}}^{0}(\theta)(\omega)\right) d P(\omega)=\int_{A} \int_{\Omega} \phi\left\{\left[T_{t_{2}}^{t_{1}}\left(T_{t_{1}}^{0}(\theta)\left(\omega^{\prime}\right)\right)\right](\omega)\right\} d P(\omega) d P\left(\omega^{\prime}\right) .
$$

The above relation follows by approximating $T_{t_{1}}(\theta)$ using simple functions ([M1], pp. 5253). Since $C$ is separable and admits uniformly continuous partitions of unity, it is easy to see that (5) holds for all open sets $B$ in $C$. By uniqueness of measure-theoretic extensions, (5) also holds for all Borel sets $B$ in $C$

The proof of the time-homogeneity statement is straightforward.

\section{The semigroup.}

In the autonomous sfde

$$
\left.\begin{array}{rl}
d x(t) & =H\left(x_{t}\right) d t+G\left(x_{t}\right) d W(t), \quad t>0 \\
x_{0} & =\eta \in C,
\end{array}\right\}
$$

suppose the coefficients $H: C \rightarrow \mathbf{R}^{d}, \quad G: C \rightarrow \mathbf{R}^{d \times m}$ are globally bounded and globally Lipschitz on $C$. 
Let $C_{b}$ be the Banach space of all bounded uniformly continuous functions $\phi: C \rightarrow \mathbf{R}$, with the sup norm

$$
\|\phi\|_{C_{b}}:=\sup _{\eta \in C}|\phi(\eta)|, \quad \phi \in C_{b}
$$

Define the operators $P_{t}: C_{b} \hookrightarrow C_{b}, t \geq 0$, on $C_{b}$ by

$$
P_{t}(\phi)(\eta):=E \phi\left({ }^{\eta} x_{t}\right), \quad t \geq 0, \phi \in C_{b}, \eta \in C
$$

For any $\phi \in C_{b}$ and any finite Borel measure $\mu$ on $C$, define the pairing

$$
<\phi, \mu>:=\int_{\eta \in C} \phi(\eta) d \mu(\eta)
$$

Say that a family $\phi_{t} \in C_{b}, t>0$, converges weakly to $\phi \in C_{b}$ as $t \rightarrow 0+$ if

$$
\lim _{t \rightarrow 0+}<\phi_{t}, \mu>=<\phi, \mu>
$$

for all finite regular Borel measures $\mu$ on $C$. In this case we write $\phi:=w-\lim _{t \rightarrow 0+} \phi_{t}$. This is equivalent to the following

$$
\left\{\begin{array}{l}
\phi_{t}(\eta) \rightarrow \phi(\eta) \text { as } t \rightarrow 0+, \text { for all } \eta \in C, \text { and } \\
\left\{\left\|\phi_{t}\right\|_{C_{b}}: t \geq 0\right\} \text { is bounded. }
\end{array}\right.
$$

The proof of this equivalence uses the uniform boundedness principle and the dominated convergence theorem ([Dy], Vol. 1, p. 50).

Theorem II.2. ([M1])

(i) $\left\{P_{t}\right\}_{t \geq 0}$ is a one-parameter contraction semigroup on $C_{b}$.

(ii) $\left\{P_{t}\right\}_{t \geq 0}$ is weakly continuous at $t=0$; i.e.

$$
\left\{\begin{array}{l}
P_{t}(\phi)(\eta) \rightarrow \phi(\eta) \text { as } t \rightarrow 0+, \text { and } \\
\left\{\left|P_{t}(\phi)(\eta)\right|: t \geq 0, \eta \in C\right\} \text { is bounded by }\|\phi\|_{C_{b}}
\end{array}\right.
$$

(iii) If $r>0,\left\{P_{t}\right\}_{t \geq 0}$ is never strongly continuous on $C_{b}$ under the sup norm.

Proof.

(i) The one-parameter semigroup property

$$
P_{t_{2}} \circ P_{t_{1}}=P_{t_{1}+t_{2}}, \quad t_{1}, t_{2} \geq 0
$$


follows from the continuation property (2) and the time-homogeneity of the Feller process $x_{t}$ (Theorem II.1).

(ii) The weak continuity of the semigroup $P_{t}: C_{b} \rightarrow C_{b}$, $t \geq 0$, at $t=0$ follows from the definition of $P_{t}$ and the sample-continuity of the trajectory ${ }^{\eta} x_{t}$.

(iii) To demonstrate the lack of strong continuity of the semigroup $\left\{P_{t}\right\}_{t \geq 0}$, consider the canonical shift (or "static") semigroup $S_{t}: C_{b} \rightarrow C_{b}, t \geq 0$, defined by

$$
S_{t}(\phi)(\eta):=\phi\left(\tilde{\eta}_{t}\right), \quad \phi \in C_{b}, \quad \eta \in C,
$$

where $\tilde{\eta}:[-r, \infty) \rightarrow \mathbf{R}^{d}$ is given by

$$
\tilde{\eta}(t)= \begin{cases}\eta(0), & t \geq 0, \\ \eta(t), & t \in[-r, 0) .\end{cases}
$$

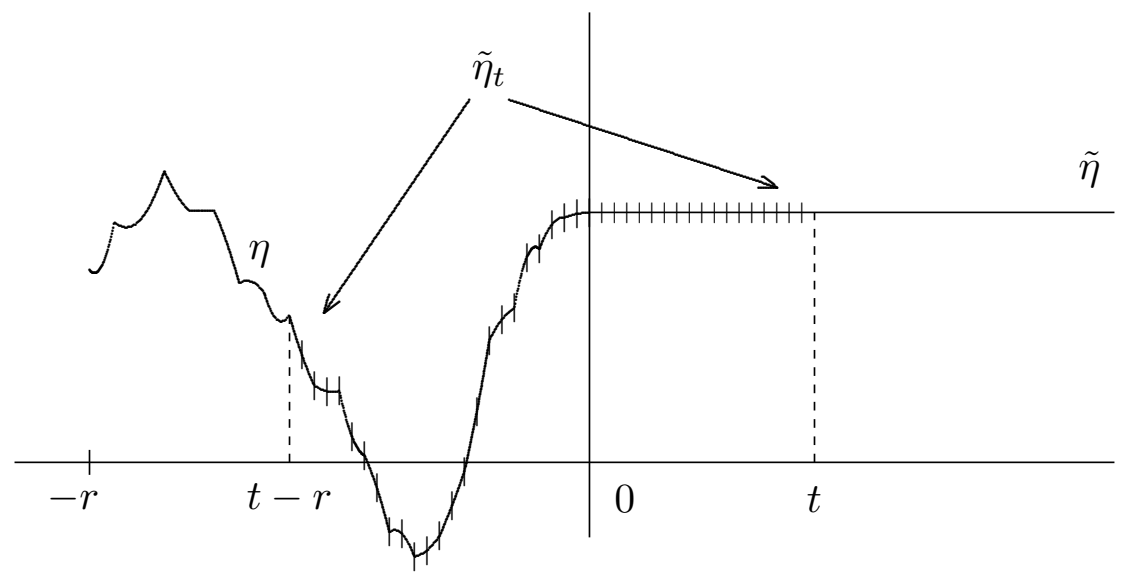

Then $\left\{P_{t}\right\}_{t \geq 0}$ is strongly continuous if and only if $\left\{S_{t}\right\}_{t \geq 0}$ is strongly continuous. That is, $\left\{P_{t}\right\}_{t \geq 0}$ and $\left\{S_{t}\right\}_{t \geq 0}$ have the same "domain of strong continuity" independently of $H, G$ and $W$. This follows from the global boundedness of $H$ and $G$ and the key relation

$$
\lim _{t \rightarrow 0+} E\left\|^{\eta} x_{t}-\tilde{\eta}_{t}\right\|_{C}^{2}=0
$$

which holds uniformly in $\eta \in C$ ([M1], Theorem IV.2.1, pp. 72-73). Now, it is not hard to see that $\left\{S_{t}\right\}_{t \geq 0}$ is strongly continuous on $C_{b}$ if and only if $C$ is locally compact. This happens if and only if $r=0$, i.e. (XIV) has no memory! ([M1], Theorems IV.2.1 and IV.2.2, pp. 72-73). The main idea for proving these equivalences is to pick any $s_{0} \in[-r, 0)$ and consider the function $\phi_{0}: C \rightarrow \mathbf{R}$ defined by

$$
\phi_{0}(\eta):= \begin{cases}\eta\left(s_{0}\right), & \|\eta\|_{C} \leq 1 \\ \frac{\eta\left(s_{0}\right)}{\|\eta\|_{C}}, & \|\eta\|_{C}>1 \\ 23 & \end{cases}
$$


Let $C_{b}^{0}$ be the domain of strong continuity of $\left\{P_{t}\right\}_{t \geq 0}$, viz.

$$
C_{b}^{0}:=\left\{\phi \in C_{b}: P_{t}(\phi) \rightarrow \phi \text { as } t \rightarrow 0+\text { in } C_{b}\right\}
$$

Let the delay $r$ be positive. Then it is easy to show that $\phi_{0} \in C_{b}$, but $\phi_{0} \notin C_{b}^{0}$.

\section{The weak infinitesimal generator.}

Define the weak generator $A: D(A) \subset C_{b} \rightarrow C_{b}$ of $\left\{P_{t}\right\}_{t \geq 0}$ by the weak limit

$$
A(\phi):=w-\lim _{t \rightarrow 0+} \frac{P_{t}(\phi)-\phi}{t},
$$

where $\phi$ belongs to the domain $D(A)$ of $A$ if and only if the above weak limit exists in $C_{b}$. Hence $D(A) \subset C_{0}^{b}$ ([Dy], Vol. 1, Chapter I, pp. 36-43). Also $D(A)$ is weakly dense in $C_{b}$ and $A$ is weakly closed. Furthermore,

$$
\frac{d}{d t} P_{t}(\phi)=A\left(P_{t}(\phi)\right)=P_{t}(A(\phi)), \quad t>0
$$

for all $\phi \in D(A)([\mathrm{Dy}]$, pp. 36-43).

Our next objective is to derive a formula for the weak generator $A$. We need to augment $C$ by adjoining a canonicald-dimensional direction. The generator $A$ will be equal to the weak generator of the shift semigroup $\left\{S_{t}\right\}_{t \geq 0}$ plus a second order linear partial differential operator along this new direction. The computation requires the following sequence of lemmas.

Let $F_{d}:=\left\{v 1_{\{0\}}: v \in \mathbf{R}^{d}\right\}$ and $C \oplus F_{d}:=\left\{\eta+v 1_{\{0\}}: \eta \in C, v \in \mathbf{R}^{d}\right\}$ with the norm $\left\|\eta+v 1_{\{0\}}\right\|:=\|\eta\|_{C}+|v|$ for $\eta \in C, v \in \mathbf{R}^{d}$.

Lemma II.1. ([M1])

Suppose $\phi: C \rightarrow \mathbf{R}$ is $C^{2}$ and $\eta \in C$. Then the Fréchet derivatives $D \phi(\eta)$ and $D^{2} \phi(\eta)$ have unique weakly continuous linear and bilinear extensions

$$
\overline{D \phi(\eta)}: C \oplus F_{d} \rightarrow \mathbf{R}, \quad \overline{D^{2} \phi(\eta)}:\left(C \oplus F_{d}\right) \times\left(C \oplus F_{d}\right) \rightarrow \mathbf{R}
$$

respectively.

Proof.

Using coordinates, it is sufficient to consider the one-dimensional case $d=1$. 
Let $\alpha \in C^{*}=[C([-r, 0], \mathbf{R})]^{*}$. We will show that there is a unique weakly continuous linear extension $\bar{\alpha}: C \oplus F_{1} \rightarrow \mathbf{R}$ of $\alpha$; viz. if $\left\{\xi^{k}\right\}$ is a bounded sequence in $C$ such that $\xi^{k}(s) \rightarrow \xi(s)$ as $k \rightarrow \infty$ for all $s \in[-r, 0]$, where $\xi \in C \oplus F_{1}$, then $\alpha\left(\xi^{k}\right) \rightarrow \bar{\alpha}(\xi)$ as $k \rightarrow \infty$. By the Riesz representation theorem there is a unique finite (regular) Borel measure $\mu$ on $[-r, 0]$ such that

$$
\alpha(\eta)=\int_{-r}^{0} \eta(s) d \mu(s)
$$

for all $\eta \in C$. Define $\bar{\alpha} \in\left[C \oplus F_{1}\right]^{*}$ by

$$
\bar{\alpha}\left(\eta+v 1_{\{0\}}\right)=\alpha(\eta)+v \mu(\{0\}), \quad \eta \in C, \quad v \in \mathbf{R} .
$$

An easy application of Lebesgue's dominated convergence theorem shows that $\bar{\alpha}$ is weakly continuous. The weak extension $\bar{\alpha}$ is unique because for any $v \in \mathbf{R}$, the function $v 1_{\{0\}}$ can be approximated weakly by a sequence of continuous functions $\left\{\xi_{0}^{k}\right\}$ where

$$
\xi_{0}^{k}(s):=\left\{\begin{array}{l}
(k s+1) v, \quad-\frac{1}{k} \leq s \leq 0 \\
0, \quad-r \leq s<-\frac{1}{k}
\end{array}\right.
$$

See the figure below.

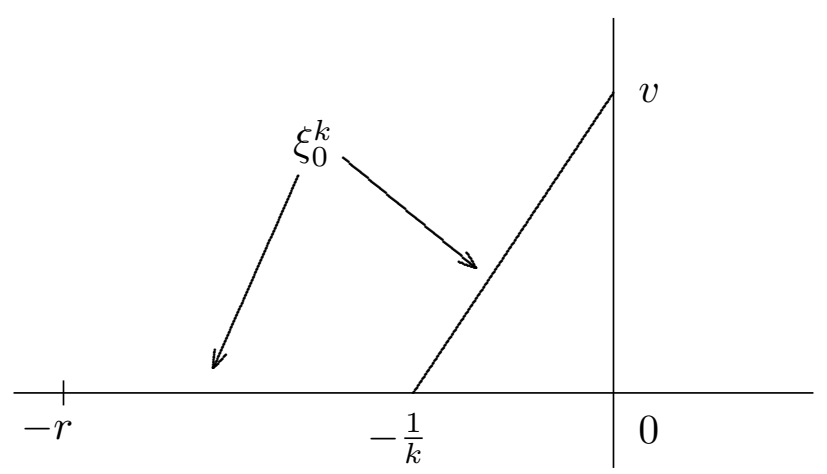

The first assertion of the lemma now follows by taking $\alpha=D \phi(\eta)$.

To prove the second assertion of the lemma, we will construct a weakly continuous bilinear extension $\bar{\beta}:\left(C \oplus F_{1}\right) \times\left(C \oplus F_{1}\right) \rightarrow \mathbf{R}$ for any continuous bilinear form $\beta: C \times C \rightarrow \mathbf{R}$. We do this by appealing to the classical theory of vector measures ([DS], I.6.3). Think of $\beta$ as a continuos linear map $C \rightarrow C^{*}$. Since $C^{*}$ is weakly complete ([DS], I.13.22, p. 341), then $\beta$ is a weakly compact linear operator ([DS], Theorem I.7.6, p. 494), viz. it maps norm-bounded sets in $C$ into weakly sequentially compact sets in $C^{*}$. Therefore there is a (unique) $C^{*}$-valued measure $\lambda$ on $[-r, 0]$ such that

$$
\beta(\xi)=\int_{-r}^{0} \xi(s) d \lambda(s)
$$


for all $\xi \in C$ ([DS], Theorem VI.7.3, p. 493). By the dominated convergence theorem for vector measures ([DS], Theorem IV.10.10, p. 328), one could approximate elements in $F_{1}$ by weakly convergent sequences of type $\left\{\xi_{0}^{k}\right\}$ above. This gives a unique weakly continuous linear extension $\hat{\beta}: C \oplus F_{1} \rightarrow C^{*}$ of $\beta$. Next for each $\eta \in C, v \in \mathbf{R}$, extend $\hat{\beta}\left(\eta+v 1_{\{0\}}\right) \in C^{*}$ to a weakly continuous linear map $\overline{\hat{\beta}\left(\eta+v 1_{\{0\}}\right)}: C \oplus F_{1} \rightarrow \mathbf{R}$. Thus $\bar{\beta}$ corresponds to the weakly continuous bilinear extension $\overline{\hat{\beta}(\cdot)}(\cdot):\left[C \oplus F_{1}\right] \times\left[C \oplus F_{1}\right] \rightarrow \mathbf{R}$ of $\beta$.

Finally, we use $\beta=D^{2} \phi(\eta)$ for each fixed $\eta \in C$ to get the required weakly continuous bilinear extension $\overline{D^{2} \phi(\eta)}$.

\section{Lemma II.2.}

For each $t>0$ define $W_{t}^{*} \in C$ by

$$
W_{t}^{*}(s):=\left\{\begin{array}{l}
\frac{1}{\sqrt{t}}[W(t+s)-W(0)], \quad-t \leq s<0, \\
0, \quad-r \leq s \leq-t .
\end{array}\right.
$$

Let $\beta$ be a continuous bilinear form on $C$. Then

$$
\lim _{t \rightarrow 0+}\left[\frac{1}{t} E \beta\left({ }^{\eta} x_{t}-\tilde{\eta}_{t},{ }^{\eta} x_{t}-\tilde{\eta}_{t}\right)-E \beta\left(G(\eta) \circ W_{t}^{*}, G(\eta) \circ W_{t}^{*}\right)\right]=0
$$

Proof.

First observe that

$$
\lim _{t \rightarrow 0+} E\left\|\frac{1}{\sqrt{t}}\left({ }^{\eta} x_{t}-\tilde{\eta}_{t}\right)-G(\eta) \circ W_{t}^{*}\right\|_{C}^{2}=0 .
$$

The above limit follows from the Lipschitz continuity of $H$ and $G$ and the martingale properties of the Itô integral. The conclusion of the lemma follows from the bilinearity of $\beta$, Hölder's inequality and the above limit ([M1]).

Lemma II.3. ([M1])

Let $\beta$ be a continuous bilinear form on $C$ and $\left\{e_{i}\right\}_{i=1}^{m}$ be any basis for $\mathbf{R}^{m}$. Then

$$
\lim _{t \rightarrow 0+} \frac{1}{t} E \beta\left({ }^{\eta} x_{t}-\tilde{\eta}_{t},{ }^{\eta} x_{t}-\tilde{\eta}_{t}\right)=\sum_{i=1}^{m} \bar{\beta}\left(G(\eta)\left(e_{i}\right) 1_{\{0\}}, G(\eta)\left(e_{i}\right) 1_{\{0\}}\right)
$$

for each $\eta \in C$. 
Proof.

By taking coordinates, we may and will assume without loss of generality that $d=m=1$. In view of Lemma II.2, we need only show that

$$
\lim _{t \rightarrow 0+} E \beta\left(W_{t}^{*}, W_{t}^{*}\right)=\bar{\beta}\left(1_{\{0\}}, 1_{\{0\}}\right),
$$

where $W$ is one-dimensional Brownian motion and $W_{t}^{*}$ is defined in Lemma II.2. To prove the above relation we will use the following argument. Let $C \hat{\otimes}_{\pi} C$ denote the complete projective tensor product of $C$ with itself. This allows us to view the continuous bilinear form $\beta$ as a continuous linear functional $\tilde{\beta}: C \hat{\otimes}_{\pi} C \rightarrow \mathbf{R}$. Therefore, using Bochner expectation and Mercer's theorem, we have

$$
E \beta\left(W_{t}^{*}, W_{t}^{*}\right)=\tilde{\beta}\left(K_{t}\right)
$$

where

$$
K_{t}:=E\left[W_{t}^{*} \otimes W_{t}^{*}\right]=\sum_{k=0}^{\infty} \frac{8}{\pi^{2}(2 k+1)^{2}} \xi_{k}^{t} \otimes \xi_{k}^{t}, \quad \xi_{k}^{t}(s):=\cos \left\{\frac{(2 k+1) \pi s}{2 t}\right\} 1_{[-t, 0]}(s),
$$

for $s \in[-r, 0], k \geq 0$, ([M1], pp. 88-94). Taking limits as $t \rightarrow 0+$ in the above relations immediately gives the required result.

Theorem II.3. ([M1])

In (XIV), suppose $H$ and $G$ are globally bounded and Lipschitz. Let $S: D(S) \subset$ $C_{b} \rightarrow C_{b}$ be the weak generator of $\left\{S_{t}\right\}_{t \geq 0}$. Suppose $\phi$ belongs to the domain $D(S)$ of $S$ and is sufficiently smooth (e.g. $\phi$ is $C^{2}, D \phi, D^{2} \phi$ are globally bounded and Lipschitz). Then $\phi \in D(A)$, and for each $\eta \in C$,

$$
A(\phi)(\eta)=S(\phi)(\eta)+\overline{D \phi(\eta)}\left(H(\eta) 1_{\{0\}}\right)+\frac{1}{2} \sum_{i=1}^{m} \overline{D^{2} \phi(\eta)}\left(G(\eta)\left(e_{i}\right) 1_{\{0\}}, G(\eta)\left(e_{i}\right) 1_{\{0\}}\right)
$$

where $\left\{e_{i}\right\}_{i=1}^{m}$ is any basis for $\mathbf{R}^{m}$.

Proof.

We break the proof up into three steps.

Step 1.

Fix $\eta \in C$. By Taylor's theorem

$$
\phi\left({ }^{\eta} x_{t}\right)-\phi(\eta)=\phi\left(\tilde{\eta}_{t}\right)-\underset{27}{\phi(\eta)}+\underset{D \phi}{D\left(\tilde{\eta}_{t}\right)\left({ }^{\eta} x_{t}-\tilde{\eta}_{t}\right)+R(t)}
$$


a.s. for $t>0$, where

$$
R(t):=\int_{0}^{1}(1-u) D^{2} \phi\left[\tilde{\eta}_{t}+u\left({ }^{\eta} x_{t}-\tilde{\eta}_{t}\right)\right]\left({ }^{\eta} x_{t}-\tilde{\eta}_{t},{ }^{\eta} x_{t}-\tilde{\eta}_{t}\right) d u .
$$

Taking expectations and dividing by $t>0$ gives

$$
\frac{1}{t} E\left[\phi\left({ }^{\eta} x_{t}\right)-\phi(\eta)\right]=\frac{1}{t}\left[S_{t}(\phi)(\eta)-\phi(\eta)\right]+D \phi\left(\tilde{\eta}_{t}\right)\left\{E\left[\frac{1}{t}\left({ }^{\eta} x_{t}-\tilde{\eta}_{t}\right)\right]\right\}+\frac{1}{t} E R(t)
$$

for $t>0$. Since $\phi \in D(S)$, the first term on the right hand side of $(3)$ converges to $S(\phi)(\eta)$ as $t \rightarrow 0+$.

Step 2.

Consider the second term on the right hand side of (3). Then

$$
\begin{aligned}
\lim _{t \rightarrow 0+}\left[E\left\{\frac{1}{t}\left({ }^{\eta} x_{t}-\tilde{\eta}_{t}\right)\right\}\right](s) & =\left\{\begin{array}{l}
\lim _{t \rightarrow 0+} \frac{1}{t} \int_{0}^{t} E\left[H\left({ }^{\eta} x_{u}\right)\right] d u, \quad s=0 \\
0, \quad-r \leq s<0
\end{array}\right. \\
& =\left[H(\eta) 1_{\{0\}}\right](s), \quad-r \leq s \leq 0 .
\end{aligned}
$$

Since $H$ is globally bounded, then $\left\|E\left\{\frac{1}{t}\left({ }^{\eta} x_{t}-\tilde{\eta}_{t}\right)\right\}\right\|_{C}$ is bounded in $t>0$ and $\eta \in C$. Hence

$$
w-\lim _{t \rightarrow 0+}\left[E\left\{\frac{1}{t}\left({ }^{\eta} x_{t}-\tilde{\eta}_{t}\right)\right\}\right]=H(\eta) 1_{\{0\}} \quad(\notin C) .
$$

Therefore, by Lemma II.1 and the continuity of $D \phi$ at $\eta$, we get

$$
\begin{aligned}
\lim _{t \rightarrow 0+} D \phi\left(\tilde{\eta}_{t}\right)\left\{E\left[\frac{1}{t}\left({ }^{\eta} x_{t}-\tilde{\eta}_{t}\right)\right]\right\} & =\lim _{t \rightarrow 0+} D \phi(\eta)\left\{E\left[\frac{1}{t}\left({ }^{\eta} x_{t}-\tilde{\eta}_{t}\right)\right]\right\} \\
& =\overline{D \phi(\eta)}\left(H(\eta) 1_{\{0\}}\right) .
\end{aligned}
$$

Step 3.

Finally we compute the limit as $t \rightarrow 0+$ of the third term in the right-hand side of (3). Use the martingale property of the Itô integral and the Lipschitz continuity of $D^{2} \phi$ to obtain the following estimates:

$$
\begin{aligned}
\mid \frac{1}{t} E D^{2} \phi\left[\tilde{\eta}_{t}\right. & \left.+u\left({ }^{\eta} x_{t}-\tilde{\eta}_{t}\right)\right]\left({ }^{\eta} x_{t}-\tilde{\eta}_{t},{ }^{\eta} x_{t}-\tilde{\eta}_{t}\right)-\frac{1}{t} E D^{2} \phi(\eta)\left({ }^{\eta} x_{t}-\tilde{\eta}_{t},{ }^{\eta} x_{t}-\tilde{\eta}_{t}\right) \mid \\
& \leq\left(E\left\|D^{2} \phi\left[\tilde{\eta}_{t}+u\left({ }^{\eta} x_{t}-\tilde{\eta}_{t}\right)\right]-D^{2} \phi(\eta)\right\|^{2}\right)^{1 / 2}\left[\frac{1}{t^{2}} E\left\|{ }^{\eta} x_{t}-\tilde{\eta}_{t}\right\|^{4}\right]^{1 / 2} \\
& \leq K\left(t^{2}+1\right)^{1 / 2}\left(E\left\|D^{2} \phi\left[\tilde{\eta}_{t}+u\left({ }^{\eta} x_{t}-\tilde{\eta}_{t}\right)\right]-D^{2} \phi(\eta)\right\|^{2}\right)^{1 / 2}
\end{aligned}
$$


where $K$ is a positive constant independent of $u, t \in \mathbf{R}^{+}$and $\eta \in C$. The last expression tends to 0 as $t \rightarrow 0+$, uniformly for $u \in[0,1]$. Therefore by Lemma II.3,

$$
\begin{aligned}
\lim _{t \rightarrow 0+} \frac{1}{t} E R(t) & =\int_{0}^{1}(1-u) \lim _{t \rightarrow 0+} \frac{1}{t} E D^{2} \phi(\eta)\left({ }^{\eta} x_{t}-\tilde{\eta}_{t},{ }^{\eta} x_{t}-\tilde{\eta}_{t}\right) d u \\
& =\frac{1}{2} \sum_{i=1}^{m} \overline{D^{2} \phi(\eta)}\left(G(\eta)\left(e_{i}\right) 1_{\{0\}}, G(\eta)\left(e_{i}\right) 1_{\{0\}}\right) .
\end{aligned}
$$

The above is a weak limit because $\phi \in D(S)$ and has first and second derivatives globally bounded on $C$.

\section{Quasitame functions.}

Recall that a function $\phi: C \rightarrow \mathbf{R}$ is said to be tame (or is a cylinder function) if there is a finite set $\left\{s_{1}<s_{2}<\cdots<s_{k}\right\}$ in $[-r, 0]$ and a $C^{\infty}$-bounded function $f:\left(\mathbf{R}^{d}\right)^{k} \rightarrow \mathbf{R}$ such that

$$
\phi(\eta)=f\left(\eta\left(s_{1}\right), \cdots, \eta\left(s_{k}\right)\right), \quad \eta \in C .
$$

The set of all tame functions is a weakly dense subalgebra of $C_{b}$, invariant under the static semigroup $\left\{S_{t}\right\}_{t \geq 0}$, and generates Borel $C$. For each $k \geq 2$ the tame function $\phi$ lies outside the domain of strong continuity $C_{b}^{0}$ of $\left\{P_{t}\right\}_{t \geq 0}$, and hence outside $D(A)$ ([M1], pp. 98-103). See also the proof of Theorem IV.2.2 in ([M1], pp. 73-76). To overcome this difficulty we introduce the following definition.

\section{Definition.}

Say $\phi: C \rightarrow \mathbf{R}$ is quasitame if there are $C^{\infty}$-bounded maps $h:\left(\mathbf{R}^{d}\right)^{k} \rightarrow \mathbf{R}, f_{j}$ : $\mathbf{R}^{d} \rightarrow \mathbf{R}^{d}$, and piecewise $C^{1}$ functions $g_{j}:[-r, 0] \rightarrow \mathbf{R}, 1 \leq j \leq k-1$, such that

$$
\phi(\eta)=h\left(\int_{-r}^{0} f_{1}(\eta(s)) g_{1}(s) d s, \cdots, \int_{-r}^{0} f_{k-1}(\eta(s)) g_{k-1}(s) d s, \eta(0)\right)
$$

for all $\eta \in C$.

Theorem II.4. ([M1])

The set of all quasitame functions is a weakly dense subalgebra of $C_{b}^{0}$, invariant under $\left\{S_{t}\right\}_{t \geq 0}$, generates Borel $C$ and belongs to $D(A)$. In particular, if $\phi$ is the quasitame function given by (4), then

$$
\left.\begin{array}{c}
A(\phi)(\eta)=\sum_{j=1}^{k-1} D_{j} h(m(\eta))\left\{f_{j}(\eta(0)) g_{j}(0)-f_{j}(\eta(-r)) g_{j}(-r)-\int_{-r}^{0} f_{j}(\eta(s)) g_{j}^{\prime}(s) d s\right\} \\
\quad+D_{k} h(m(\eta))(H(\eta))+\frac{1}{2} \operatorname{trace}\left[D_{k}^{2} h(m(\eta)) \circ(G(\eta) \times G(\eta))\right]
\end{array}\right\}
$$


for all $\eta \in C$, where

$$
m(\eta):=\left(\int_{-r}^{0} f_{1}(\eta(s)) g_{1}(s) d s, \cdots, \int_{-r}^{0} f_{k-1}(\eta(s)) g_{k-1}(s) d s, \eta(0)\right) .
$$

Proof.

Let $S: D(S) \subset C_{b} \rightarrow C_{b}$ be the weak generator of $\left\{S_{t}\right\}_{t \geq 0}$. An elementary computation shows that every quasitame function $\phi$ belongs to $D(S) \subset C_{b}^{0}$ and

$$
S(\phi)(\eta)=\sum_{j=1}^{k-1} D_{j} h(m(\eta))\left\{f_{j}(\eta(0)) g_{j}(0)-f_{j}(\eta(-r)) g_{j}(-r)-\int_{-r}^{0} f_{j}(\eta(s)) g_{j}^{\prime}(s) d s\right\}
$$

for all $\eta \in C$.

The invariance of the quasitame functions under $\left\{S_{t}\right\}_{t \geq 0}$ follows directly from the definition of a quasitame function and that of $\left\{S_{t}\right\}_{t \geq 0}$.

It is easy to check that the set of quasitame functions is closed under addition and multiplication of functions in $C_{b}$.

Each tame function is a weak limit of a sequence of quasitame functions. Since the tame functions are dense in $C_{b}$ and generate Borel $C$, then so do the quasitame functions.

Formula (5) follows from Theorem II.3 and (7). Alternatively, one could use Itô's formula directly to obtain (5).

\section{Remarks.}

(i) In Theorem II.4, the space $C$ may be replaced by the Hilbert space $M_{2}$. In this case, there is no need for the weak extensions because $M_{2}$ is weakly complete. Extensions of $D \phi(v, \eta)$ and $D^{2} \phi(v, \eta)$ correspond to partial derivatives in the $\mathbf{R}^{d_{-}}$ variable. Tame functions do not exist on $M_{2}$ but quasitame functions do! (with $\eta(0)$ replaced by $\left.v \in \mathbf{R}^{d}\right)$.

(ii) An analysis of the supermartingale behavior and stability of $\phi\left({ }^{\eta} x_{t}\right)$ is given in Kushner $([\mathrm{Ku}])$. An infinite fading memory setting was developed by Mizel and Trutzer ([MT]) in a suitably weighted state space $\mathbf{R}^{d} \times L^{2}((-\infty, 0], \mathbf{R} ; \rho)$

(iii) If $\phi: C \rightarrow \mathbf{R}$ is a quasitame function, then the process $\phi\left({ }^{\eta} x_{t}\right)$ is a semimartingale, and the following Itô formula holds

$$
d\left[\phi\left({ }^{\eta} x_{t}\right)\right]=A(\phi)\left({ }^{\eta} x_{t}\right) d t+\overline{D \phi\left({ }^{\eta} x_{t}\right)}\left(H\left({ }^{\eta} x_{t}\right) 1_{\{0\}}\right) d W(t) .
$$

It is interesting to note here that this formula holds in spite of the fact the trajectory ${ }^{\eta} x_{t}$ is not known to be a semimartingale. 


\section{Chapter III}

\section{Regularity and Classification of SFDE's}

In this chapter, we will discuss the regularity of the trajectory random field of the sfde

$$
\left.\begin{array}{rl}
d x(t) & =H\left(t, x_{t}\right) d t+G\left(t, x_{t}\right) d W(t), \quad t>0 \\
x_{0} & =\eta \in C .
\end{array}\right\}
$$

The trajectory field $X(t, \eta, \omega):=\left\{{ }^{\eta} x_{t}(\omega): t \geq 0, \eta \in C\right\}$ will be viewed as a mapping of the three variables $(t, \eta, \omega)$, and its regularity in each of the variables will be analyzed. In the time variable, we will investigate $\alpha$-Hölder continuity of $X(t, \eta, \omega)$ for times $t$ greater than the delay $r$. The almost sure (pathwise) dependence of $X(t, \eta, \omega)$ on the initial state $\eta$ is counterintuitive. We will show that for the discrete delay case

$$
d x(t)=x(t-r) d W(t)
$$

the trajectory $X$ is locally unbounded and non-linear in the initial variable $\eta$. This pathological behavior leads to a classification of sfde's into regular and singular types.

We then give sufficient conditions for regularity of linear sfde's driven by white noise or semimartingales. A complete characterization of regular linear sfde's is not known.

A regular Sussman-Doss class of nonlinear sfde's is introduced. Here we show the existence of a non-linear semiflow which carries bounded sets into relatively compact ones.

Denote the state space by $E$ where $E=C$ or $M_{2}:=\mathbf{R}^{d} \times L^{2}\left([-r, 0], \mathbf{R}^{d}\right)$. Most results in this chapter hold for either choice of state space.

For $\alpha \in(0,1)$ denote by $C^{\alpha}:=C^{\alpha}\left([-r, 0], \mathbf{R}^{d}\right)$ the separable Banach space of $\alpha$ Hölder continuous paths $\eta:[-r, 0] \rightarrow \mathbf{R}^{d}$ obtained as the completion of the space of smooth paths $C^{\infty}\left([-r, 0], \mathbf{R}^{d}\right)$ in the $\alpha$-Hölder norm

$$
\|\eta\|_{\alpha}:=\|\eta\|_{C}+\sup \left\{\frac{\left|\eta\left(s_{1}\right)-\eta\left(s_{2}\right)\right|}{\left|s_{1}-s_{2}\right|^{\alpha}}: s_{1}, s_{2} \in[-r, 0], s_{1} \neq s_{2}\right\} .
$$

([FT], $[\mathrm{Tr}])$. The separability of $C^{\alpha}$ will be needed in order to establish the existence of measurable versions of the trajectory field.

\section{Measurable versions and regularity in distribution.}

Our first step is to think of ${ }^{\eta} x_{t}(\omega)$ as a measurable mapping $X: \mathbf{R}^{+} \times C \times \Omega \rightarrow C$ in the three variables $(t, \eta, \omega)$ simultaneously: 
Theorem III.1. ([M1])

In the sfde

$$
\left.\begin{array}{rl}
d x(t) & =H\left(t, x_{t}\right) d t+G\left(t, x_{t}\right) d W(t), \quad t>0 \\
x_{0} & =\eta \in C,
\end{array}\right\}
$$

assume that the coefficients $H:[0, \infty) \times C \rightarrow \mathbf{R}^{d}$ and $G:[0, \infty) \times C \rightarrow \mathbf{R}^{d \times m}$ are (jointly) continuous and globally Lipschitz in the second variable uniformly with respect to $t$ in compact sets of $[0, \infty)$. Then the following statements are true:

(i) For any $0<\alpha<\frac{1}{2}$ and each initial path $\eta \in C$,

$$
P\left({ }^{\eta} x_{t} \in C^{\alpha}, \text { for all } t \geq r\right)=1 .
$$

(ii) The trajectory field ${ }^{\eta} x_{t}, t \geq 0, \eta \in C$, has a measurable version

$$
X: \mathbf{R}^{+} \times C \times \Omega \rightarrow C .
$$

(iii) The trajectory field ${ }^{\eta} x_{t}, t \geq r, \eta \in C$, admits a measurable version

$$
[r, \infty) \times C \times \Omega \rightarrow C^{\alpha} .
$$

\section{Remark.}

Similar statements hold when the state space $E=M_{2}$.

Consider the space $L^{0}(\Omega, E)$ with the complete (pseudo)metric

$$
d_{E}\left(\theta_{1}, \theta_{2}\right):=\inf _{\epsilon>0}\left[\epsilon+P\left(\left\|\theta_{1}-\theta_{2}\right\|_{E} \geq \epsilon\right)\right], \quad \theta_{1}, \theta_{2} \in L^{0}(\Omega, E) .
$$

This pseudometric corresponds to convergence in probability ([DS], Lemma III.2.7, p. 104). Proof of Theorem III.1.

Fix any $\alpha \in(0,1 / 2)$.

(i) It is sufficient to show that

$$
P\left({ }^{\eta} x \mid[0, a] \in C^{\alpha}\left([0, a], \mathbf{R}^{d}\right)\right)=1
$$

for any positive real $a$. This follows from the Borel-Cantelli lemma and the estimate

$$
P\left(\sup _{0 \leq t_{1}, t_{2} \leq a, t_{1} \neq t_{1}} \frac{\left|\eta x\left(t_{1}\right)-{ }^{\eta} x\left(t_{2}\right)\right|}{\left|t_{1}-t_{2}\right|^{\alpha}} \geq N\right) \leq C_{k}^{1}\left(1+\|\eta\|_{C}^{2 k}\right) \frac{1}{N^{2 k}},
$$


for all integers $k>(1-2 \alpha)^{-1}$. In the above estimate, $C_{k}^{1}$ is a positive constant independent of $\eta \in C$ but may depend on $k, \alpha, m, d, a$. The above estimate may be proved using Gronwall's lemma, Chebyshev's inequality, and a Garsia-RodemichRumsey lemma ([GRR], [M1], Theorem 4.1, p. 150; Theorem 4.4, pp. 152-154).

(ii) In view of Remark (v) following the proof of Theorem I.2, the trajectory

$$
\begin{aligned}
{[0, a] \times C } & \rightarrow L^{2}(\Omega, C) \subset L^{0}(\Omega, C) \\
(t, \eta) & \mapsto{ }^{\eta} x_{t}
\end{aligned}
$$

is globally Lipschitz in $\eta$ uniformly with respect to $t$ in compact sets, and is continuous in $t$ for fixed $\eta$ ([M1], Theorem 3.1, p. 41). Therefore it is jointly continuous in $(t, \eta)$ as a map

$$
[0, a] \times C \ni(t, \eta) \mapsto{ }^{\eta} x_{t} \in L^{0}(\Omega, C) .
$$

We now apply the Cohn-Hoffman-J申rgensen Theorem:

If $T, E$ are complete separable metric spaces, then each Borel map $X: T \rightarrow$ $L^{0}(\Omega, E ; \mathcal{F})$ admits a measurable version $T \times \Omega \rightarrow E \quad([\mathrm{M} 1]$, p. 16).

By this theorem, the trajectory field has a version $X(t, \eta, \omega):={ }^{\eta} x_{t}(\cdot, \omega)$ which is jointly measurable in $(t, \eta, \omega)$. To see this, just take $T=[0, a] \times C, E=C$ in the Cohn-Hoffman-J $\phi$ rgensen Theorem.

(iii) The estimate

$$
P\left(\left\|^{\eta_{1}} x_{t}-{ }^{\eta_{2}} x_{t}\right\|_{C^{\alpha}} \geq N\right) \leq \frac{C_{k}^{2}}{N^{2 k}}\left\|\eta_{1}-\eta_{2}\right\|_{C}^{2 k}
$$

for $t \in[r, a], N>0$, ([M1], Theorem 4.7, pp. 158-162) may be used to prove joint continuity of the trajectory field

$$
\begin{aligned}
{[r, a] \times C } & \rightarrow L^{0}\left(\Omega, C^{\alpha}\right) \\
(t, \eta) & \mapsto{ }^{\eta} x_{t}
\end{aligned}
$$

when viewed as a process with values in the separable Banach space $C^{\alpha}([\mathrm{M} 1]$, Theorem 4.7, pp. 158-162). Again apply the Cohn-Hoffman-J $\phi$ rgensen theorem.

As we have seen in Chapter I, the trajectory of a sfde possesses good regularity properties in the mean-square. The following theorem shows good behavior in distribution. 
Theorem III.2. ([M1])

Suppose the coefficients $H$ and $G$ are globally Lipschitz in the second variable uniformly with respect to the first. Let $\alpha \in(0,1 / 2)$ and $k$ be any integer such that $k>(1-2 \alpha)^{-1}$. Then there are positive constants $C_{k}^{i}:=C_{k}^{i}(\alpha, k, m, d, a), i=3,4,5$ such that

$$
\begin{gathered}
d_{C}\left({ }^{\eta_{1}} x_{t},{ }^{\eta_{2}} x_{t}\right) \leq C_{k}^{3}\left\|\eta_{1}-\eta_{2}\right\|_{C}^{2 k /(2 k+1)}, \quad t \in[0, a], \\
d_{C^{\alpha}}\left({ }^{\eta_{1}} x_{t},{ }^{\eta_{2}} x_{t}\right) \leq C_{k}^{4}\left\|\eta_{1}-\eta_{2}\right\|_{C}^{2 k /(2 k+1)}, \quad t \in[r, a], \\
P\left(\|\|^{\eta} x_{t} \|_{C^{\alpha}} \geq N\right) \leq C_{k}^{5}\left(1+\|\eta\|_{C}^{2 k}\right) \frac{1}{N^{2 k}}, \quad t \in[r, a], \quad N>0 .
\end{gathered}
$$

In particular, the transition probabilities

$$
\begin{array}{r}
{[r, a] \times C \rightarrow \mathcal{M}_{p}(C)} \\
(t, \eta) \mapsto p(0, \eta, t, \cdot)
\end{array}
$$

take bounded sets in $[r, a] \times C$ into relatively weak* compact sets in the space $\mathcal{M}_{p}(C)$ of probability measures on $C$.

Proof.

The proofs of the three estimates use Gronwall's lemma, Chebyshev's inequality, and the Garsia-Rodemich-Rumsey lemma ([GRR], [M1], Theorem 4.1, p. 150; Theorem 4.7, pp. 159-162). The weak* compactness assertion follows from the third estimate, Prohorov's theorem and the compactness of the embedding $C^{\alpha} \hookrightarrow C$ ([M1], Theorem 4.6, pp. 156-158).

\section{Erratic behavior. The noisy feedback loop revisited.}

In this section, we will show that trajectories of certain types of sfde's exhibit highly erratic pathwise dependence on the initial path. We start with a definition.

\section{Definition.}

A sfde is regular with respect to $M_{2}$ if its trajectory random field $\left\{\left(x(t), x_{t}\right)\right.$ : $\left.\left(x(0), x_{0}\right)=(v, \eta) \in M_{2}, t \geq 0\right\}$ admits a $\left(\right.$ Borel $\mathbf{R}^{+} \otimes$ Borel $M_{2} \otimes \mathcal{F}$, Borel $\left.M_{2}\right)$-measurable version $X: \mathbf{R}^{+} \times M_{2} \times \Omega \rightarrow M_{2}$ with almost all sample functions continuous on $\mathbf{R}^{+} \times M_{2}$. The sfde is said to be singular otherwise. Regularity with respect to $C$ is similarly defined. 
An example of a singular sfde is the one-dimensional linear sdde with a positive delay $r$ and driven by a Wiener process $W$; viz.

$$
\left.\begin{array}{rl}
d x(t) & =\sigma x(t-r)) d W(t), \quad t>0 \\
\left(x(0), x_{0}\right) & =(v, \eta) \in M_{2}:=\mathbf{R} \times L^{2}([-r, 0], \mathbf{R}) .
\end{array}\right\}
$$

Recall that the above sdde is a model for the noisy feedback loop introduced in Chapter I. Theorem III.3 below implies that (I) is singular with respect to $M_{2}$ (and $C$ ). See also [M2].

Consider the regularity of the more general one-dimensional linear sfde:

$$
\left.\begin{array}{rl}
d x(t) & =\int_{-r}^{0} x(t+s) d \nu(s) d W(t), \quad t>0 \\
\left.(0), x_{0}\right) & \in M_{2}:=\mathbf{R} \times L^{2}([-r, 0], \mathbf{R}),
\end{array}\right\}
$$

where $W$ is a Wiener process and $\nu$ is a fixed finite real-valued Borel measure on $[-r, 0]$.

Using integration by parts to "eliminate" the Itô integral, the reader may show that $\left(\mathrm{II}^{\prime}\right)$ is regular if $\nu$ has a $C^{1}$ (or even $L_{1}^{2}$ ) density with respect to Lebesgue measure on $[-r, 0]$.

The following theorem gives conditions on the measure $\nu$ under which $\left(\mathrm{II}^{\prime}\right)$ is singular.

Theorem III.3. ([MS2])

Let $r>0$, and suppose that there exists $\epsilon \in(0, r)$ such that $\operatorname{supp} \nu \subset[-r,-\epsilon]$. Suppose $0<t_{0} \leq \epsilon$. For each $k \geq 1$, set

$$
\nu_{k}:=\sqrt{t_{0}}\left|\int_{[-r, 0]} e^{2 \pi i k s / t_{0}} d \nu(s)\right|
$$

Assume that

$$
\sum_{k=1}^{\infty} \nu_{k} x^{1 / \nu_{k}^{2}}=\infty
$$

for all $x \in(0,1)$. Let $Y:[0, \epsilon] \times M_{2} \times \Omega \rightarrow \mathbf{R}$ be any Borel-measurable version of the solution field $\left\{x(t): 0 \leq t \leq \epsilon,\left(x(0), x_{0}\right)=(v, \eta) \in M_{2}\right\}$ of $\left(I I^{\prime}\right)$. Then for a.a. $\omega \in \Omega$, the map $Y\left(t_{0}, \cdot, \omega\right): M_{2} \rightarrow \mathbf{R}$ is unbounded in every neighborhood of every point in $M_{2}$, and (hence) non-linear. 
Corollary III.3.1. ([M1])

Suppose $r>0$ and $\sigma \neq 0$ in (I). Then the trajectory $\left\{{ }^{\eta} x_{t}: 0 \leq t \leq r, \eta \in C\right\}$ of (I) has a measurable version $X:[0, r] \times C \times \Omega \rightarrow C$ such that for every $t \in(0, r]$

$$
P\left(X\left(t, \eta_{1}+\lambda \eta_{2}, \cdot\right)=X\left(t, \eta_{1}, \cdot\right)+\lambda X\left(t, \eta_{2}, \cdot\right) \text { for all } \lambda \in \mathbf{R}, \text { and all } \eta_{1}, \eta_{2} \in C\right)=0
$$

but

$$
P\left(X\left(t, \eta_{1}+\lambda \eta_{2}, \cdot\right)=X\left(t, \eta_{1}, \cdot\right)+\lambda X\left(t, \eta_{2}, \cdot\right)\right)=1 .
$$

for all $\lambda \in \mathbf{R}, \eta_{1}, \eta_{2} \in C$.

\section{Remark.}

(i) Condition (1) of Theorem III.3 is implied by $\lim _{k \rightarrow \infty} \nu_{k} \sqrt{\log k}=\infty$.

(ii) For the delay equation (I), $\nu=\sigma \delta_{-r}, \epsilon=r$. In this case condition (1) is satisfied for every $t_{0} \in(0, r]$.

(iii) Theorem III.3 also holds for the state space $C$ since every bounded set in $C$ is also bounded in $L^{2}([-r, 0], \mathbf{R})$.

Proof of Theorem III.3.

This proof is joint work of V. J. Mizel and the author.

The main idea is to track the solution random field of (a complexified version of) $\left(\mathrm{II}^{\prime}\right)$ along the classical Fourier basis

$$
\eta_{k}(s)=\mathrm{e}^{2 \pi i k s / t_{0}}, \quad-r \leq s \leq 0, \quad k \geq 1
$$

in $L^{2}([-r, 0], \mathbf{C})$. On this basis, the solution field gives an infinite family of independent Gaussian random variables. This allows us to show that no Borel measurable version of the solution field can be bounded with positive probability on an arbitrarily small neighborhood of 0 in $M_{2}$, and hence on any neighborhood of any point in $M_{2}$ (cf. [M1], pp. 144-148; [M2]). For simplicity of computations, complexify the state space in (II') by allowing $(v, \eta)$ to belong to $M_{2}^{C}:=\mathbf{C} \times L^{2}([-r, 0]$, $\mathbf{C})$. Thus we consider the sfde

$$
\left.\begin{array}{rl}
d x(t) & =\int_{[-r, 0]} x(t+s) d \nu(s) d W(t), \quad t>0, \\
\left.(0), x_{0}\right) & =(v, \eta) \in M_{2}^{C},
\end{array}\right\} \quad\left(I I^{\prime}-C\right)
$$

where $x(t) \in \mathbf{C}, t \geq-r$, and $\nu, W$ are real-valued. 
We use contradiction. Let $Y:[0, \epsilon] \times M_{2} \times \Omega \rightarrow \mathbf{R}$ be any Borel-measurable version of the solution field $\left\{x(t): 0 \leq t \leq \epsilon,\left(x(0), x_{0}\right)=(v, \eta) \in M_{2}\right\}$ of (II'). Suppose, if possible, that there exists a set $\Omega_{0} \in \mathcal{F}$ of positive $P$-measure, $\left(v_{0}, \eta_{0}\right) \in M_{2}$ and a positive $\delta$ such that for all $\omega \in \Omega_{0}, Y\left(t_{0}, \cdot, \omega\right)$ is bounded on the open ball $B\left(\left(v_{0}, \eta_{0}\right), \delta\right)$ in $M_{2}$ of center $\left(v_{0}, \eta_{0}\right)$ and radius $\delta$. Define the complexification $Z(\cdot, \omega): M_{2}^{C} \rightarrow \mathbf{C}$ of $Y\left(t_{0}, \cdot, \omega\right): M_{2} \rightarrow \mathbf{R}$ by

$$
Z\left(\xi_{1}+i \xi_{2}, \omega\right):=Y\left(t_{0}, \xi_{1}, \omega\right)+i Y\left(t_{0}, \xi_{2}, \omega\right), \quad i=\sqrt{-1}
$$

for all $\xi_{1}, \xi_{2} \in M_{2}, \omega \in \Omega$. Let $\left(v_{0}, \eta_{0}\right)^{C}$ denote the complexification $\left(v_{0}, \eta_{0}\right)^{C}:=\left(v_{0}, \eta_{0}\right)+$ $i\left(v_{0}, \eta_{0}\right)$. Clearly $Z(\cdot, \omega)$ is bounded on the complex ball $B\left(\left(v_{0}, \eta_{0}\right)^{C}, \delta\right)$ in $M_{2}^{C}$ for all $\omega \in \Omega_{0}$. Define the sequence of complex random variables $\left\{Z_{k}\right\}_{k=1}^{\infty}$ by

$$
Z_{k}(\omega):=Z\left(\left(\eta_{k}(0), \eta_{k}\right), \omega\right)-\eta_{k}(0), \quad \omega \in \Omega, \quad k \geq 1 .
$$

Then

$$
Z_{k}=\int_{0}^{t_{0}} \int_{[-r,-\epsilon]} \eta_{k}(u+s) d \nu(s) d W(u), \quad k \geq 1 .
$$

Using standard properties of the Itô integral together with Fubini's theorem, we get

$$
E Z_{k} \overline{Z_{l}}=\int_{[-r,-\epsilon]} \int_{[-r,-\epsilon]} \int_{0}^{t_{0}} \eta_{k}(u+s) \overline{\eta_{l}\left(u+s^{\prime}\right)} d u d \nu(s) d \nu\left(s^{\prime}\right)=0
$$

for $k \neq l$, because

$$
\int_{0}^{t_{0}} \eta_{k}(u+s) \overline{\eta_{l}\left(u+s^{\prime}\right)} d u=0
$$

whenever $k \neq l$, for all $s, s^{\prime} \in[-r, 0]$. Furthermore

$$
\int_{0}^{t_{0}} \eta_{k}(u+s) \overline{\eta_{k}\left(u+s^{\prime}\right)} d u=t_{0} \mathrm{e}^{2 \pi i k\left(s-s^{\prime}\right) / t_{0}}
$$

for all $s, s^{\prime} \in[-r, 0]$. Hence

$$
\begin{aligned}
E\left|Z_{k}\right|^{2} & =\int_{[-r,-\epsilon]} \int_{[-r,-\epsilon]} t_{0} \mathrm{e}^{2 \pi i k\left(s-s^{\prime}\right) / t_{0}} d \nu(s) d \nu\left(s^{\prime}\right) \\
& =t_{0}\left|\int_{[-r, 0]} \mathrm{e}^{2 \pi i k s / t_{0}} d \nu(s)\right|^{2} \\
& =\nu_{k}^{2} .
\end{aligned}
$$


Now $Z(\cdot, \omega): M_{2}^{C} \rightarrow \mathbf{C}$ is bounded on $B\left(\left(v_{0}, \eta_{0}\right)^{C}, \delta\right)$ for all $\omega \in \Omega_{0}$, and $\left\|\left(\eta_{k}(0), \eta_{k}\right)\right\|=$ $\sqrt{r+1}$ for all $k \geq 1$. By the linearity property

$Z\left(\left(v_{0}, \eta_{0}\right)^{C}+\frac{\delta}{2 \sqrt{r+1}}\left(\eta_{k}(0), \eta_{k}\right), \cdot\right)=Z\left(\left(v_{0}, \eta_{0}\right)^{C}, \cdot\right)+\frac{\delta}{2 \sqrt{r+1}} Z\left(\left(\eta_{k}(0), \eta_{k}\right), \cdot\right), k \geq 1$, a.s.

it follows that

$$
P\left(\sup _{k \geq 1}\left|Z_{k}\right|<\infty\right)>0
$$

It is easy to check that $\left\{\operatorname{Re} Z_{k}, \operatorname{Im} Z_{k}: k \geq 1\right\}$ are independent $\mathcal{N}\left(0, \nu_{k}^{2} / 2\right)$ distributed Gaussian random variables. The rest of the proof is an argument due to Dudley $([\mathrm{Du}])$ which gives a contradiction to $(3)$.

For each integer $N \geq 1$, we have

$$
\begin{aligned}
P\left(\sup _{k \geq 1}\left|Z_{k}\right|<N\right) & \leq \prod_{k \geq 1} P\left(\left|\operatorname{Re} Z_{k}\right|<N\right) \\
& =\prod_{k \geq 1}\left[1-\frac{2}{\sqrt{2 \pi}} \int_{\frac{\sqrt{2} N}{\nu_{k}}}^{\infty} \mathrm{e}^{-x^{2} / 2} d x\right] \\
& \leq \exp \left\{-\frac{2}{\sqrt{2 \pi}} \sum_{k=1}^{\infty} \int_{\frac{\sqrt{2} N}{\nu_{k}}}^{\infty} \mathrm{e}^{-x^{2} / 2} d x\right\} .
\end{aligned}
$$

But there is an $N_{0}>1$ (independent of $k \geq 1$ ) such that

$$
\int_{\frac{\sqrt{2} N}{\nu_{k}}}^{\infty} \mathrm{e}^{-x^{2} / 2} d x \geq \frac{\nu_{k}}{2 \sqrt{2} N} \mathrm{e}^{-\frac{N^{2}}{\nu_{k}^{2}}}
$$

for all $N \geq N_{0}$ and all $k \geq 1$.

Combining (4) and (5) and using hypothesis (1) of the theorem, we obtain

$$
P\left(\sup _{k \geq 1}\left|Z_{k}\right|<N\right)=0
$$

for all $N \geq N_{0}$. Therefore $P\left(\sup _{k \geq 1}\left|Z_{k}\right|<\infty\right)=0$. This contradicts (3).

Since $Y\left(t_{0}, \cdot, \omega\right)$ is locally unbounded, it must be non-linear because of Douady's Theorem:

Every Borel measurable linear map between two Banach spaces is continuous. ([Sc], pp. 155-160). This completes the proof of the theorem. 
Note that the pathological phenomenon in Theorem III.3 is peculiar to the delay case $r>0$. The proof of the theorem suggests that this pathology is due to the Gaussian nature of the Wiener process $W$, coupled with the infinite-dimensionality of the state space $M_{2}$. Because of this, one may expect similar difficulties in certain types of linear spde's driven by multi-dimensional white noise ([FS]).

\section{Problem.}

Classify all finite signed Borel measures $\nu$ on $[-r, 0]$ for which $\left(I I^{\prime}\right)$ is regular.

Note that (I) automatically satisfies the conditions of Theorem III.3, and hence its trajectory field explodes on every small neighborhood of $0 \in M_{2}$. In view of this pathology, it is somewhat surprising that the maximal exponential growth rate of the trajectory of (I) is negative for small $\sigma$ and is bounded away from zero independently of the choice of the initial path in $M_{2}$. This will be shown later in Chapter V (Theorem V.1).

\section{Regularity of linear systems. White noise.}

Here we will consider the following class of linear sfde's on $\mathbf{R}^{d}$ driven by $m$ dimensional Brownian motion $W:=\left(W_{1}, \cdots, W_{m}\right)$ :

$$
\left.\begin{array}{rl}
d x(t) & =H\left(x\left(t-d_{1}\right), \cdots, x\left(t-d_{N}\right), x(t), x_{t}\right) d t+\sum_{i=1}^{m} g_{i} x(t) d W_{i}(t), \quad t>0 \\
\left(x(0), x_{0}\right) & =(v, \eta) \in M_{2}:=\mathbf{R}^{d} \times L^{2}\left([-r, 0], \mathbf{R}^{d}\right) .
\end{array}\right\}
$$

The above sfde is defined on a canonical complete filtered Wiener space $\left(\Omega, \mathcal{F},\left(\mathcal{F}_{t}\right)_{t \geq 0}, P\right)$, where:

$\Omega$ is the space of all continuous paths $\omega: \mathbf{R}^{+} \rightarrow \mathbf{R}^{m}, \omega(0)=0$, in Euclidean space $\mathbf{R}^{m}$, with the compact open topology;

$\mathcal{F}$ is the completed Borel $\sigma$-field of $\Omega$;

$P$ is Wiener measure on $\Omega$;

$\mathcal{F}_{t}$ is the completed sub- $\sigma$-field of $\mathcal{F}$ generated by the evaluations $\omega \mapsto \omega(u)$,

$0 \leq u \leq t, \quad t \geq 0$.

$d W_{i}(t)$ are Itô stochastic differentials for $1 \leq i \leq m$. The drift coefficient is a fixed continuous linear map $H:\left(\mathbf{R}^{d}\right)^{N} \times M_{2} \rightarrow \mathbf{R}^{d}$ with several finite delays $0<d_{1}<d_{2}<$ $\cdots<d_{N} \leq r$. There are no delays in the diffusion coefficient. The diffusion coefficients are fixed (deterministic) $d \times d$-matrices $g_{i}, i=1,2, \ldots, m$. 
Theorem III.4. ([M3])

The sfde (VIII) is regular with respect to the state space $M_{2}$. There is a measurable version $X: \mathbf{R}^{+} \times M_{2} \times \Omega \rightarrow M_{2}$ of the trajectory field $\left\{\left(x(t), x_{t}\right): t \in \mathbf{R}^{+},\left(x(0), x_{0}\right)=\right.$ $\left.(v, \eta) \in M_{2}\right\}$ with the following properties:

(i) For each $(v, \eta) \in M_{2}$ and $t \in \mathbf{R}^{+}, X(t,(v, \eta), \cdot)=\left(x(t), x_{t}\right)$ a.s., is $\mathcal{F}_{t^{-}}$ measurable and belongs to $L^{2}\left(\Omega, M_{2} ; P\right)$.

(ii) There exists $\Omega_{0} \in \mathcal{F}$ of full measure such that, for all $\omega \in \Omega_{0}$, the map $X(\cdot, \cdot, \omega): \mathbf{R}^{+} \times M_{2} \rightarrow M_{2}$ is continuous.

(iii) For each $t \in \mathbf{R}^{+}$and every $\omega \in \Omega_{0}$, the map $X(t, \cdot, \omega): M_{2} \rightarrow M_{2}$ is continuous linear; for each $\omega \in \Omega_{0}$, the map $\mathbf{R}^{+} \ni t \mapsto X(t, \cdot, \omega) \in L\left(M_{2}\right)$ is measurable and locally bounded in the uniform operator norm on $L\left(M_{2}\right)$. The map $[r, \infty) \ni t \mapsto X(t, \cdot, \omega) \in L\left(M_{2}\right)$ is continuous for all $\omega \in \Omega_{0}$.

(iv) For each $t \geq r$ and all $\omega \in \Omega_{0}$, the map

$$
X(t, \cdot, \omega): M_{2} \rightarrow M_{2}
$$

is compact.

The proof uses a variational technique to reduce the problem to the solution of a random family of classical integral equations involving no stochastic integrals ([M3]).

The compactness of the semi-flow for $t \geq r$ will be used later to define the notion of hyperbolicity for the sfde (VIII) and the associated exponential dichotomies. See Chapter IV.

\section{Regularity of linear systems. Semimartingale noise.}

Let $\left(\Omega, \mathcal{F},\left(\mathcal{F}_{t}\right)_{t \geq 0}, P\right)$ be a complete filtered probability space satisfying the usual conditions. Consider the following linear sfde:

$$
\begin{aligned}
& \left.d x(t)=\left\{\int_{[-r, 0]} \nu(t)(d s) x(t+s)\right\} d t+d N(t) \int_{-r}^{0} K(t)(s) x(t+s) d s\right\} \\
& +d L(t) x(t-), \quad t>0 \\
& \left(x(0), x_{0}\right)=(v, \eta) \in M_{2}:=\mathbf{R}^{d} \times L^{2}\left([-r, 0], \mathbf{R}^{d}\right) .
\end{aligned}
$$

The basic set-up and hypotheses underlying (IX) are described below. Let $\mathbf{R}^{d \times d}$ denote the Euclidean space of all $d \times d$-matrices. Denote by $\mathcal{M}\left([-r, 0], \mathbf{R}^{d \times d}\right)$ the space of all $\mathbf{R}^{d \times d}$-valued Borel measures on $[-r, 0]$ (or $R^{d \times d}$-valued functions of bounded variation on $[-r, 0])$. This space is given the $\sigma$-algebra generated by all evaluations. In the sfde (IX), 
all vectors are considered as column vectors. The noise in (IX) is provided by $\mathbf{R}^{d \times d}$-valued $\left(\mathcal{F}_{t}\right)_{t \geq 0^{-}}$semimartingales $N$ and $L$. The memory is driven by a measure-valued process $\nu: \mathbf{R} \times \Omega \rightarrow \mathcal{M}\left([-r, 0], \mathbf{R}^{d \times d}\right)$ and a "smooth" process $K$. More specifically, we impose the following hypotheses.

\section{Hypotheses (R)}

(i) The process $\nu: \mathbf{R} \times \Omega \rightarrow \mathcal{M}\left([-r, 0], \mathbf{R}^{d \times d}\right)$ is measurable and $\left(\mathcal{F}_{t}\right)_{t \geq 0}$-adapted. For each $\omega \in \Omega$ and $t \geq 0$, define the positive measure $\bar{\nu}(t, \omega)$ on $[-r, \infty)$ by

$$
\bar{\nu}(t, \omega)(A):=|\nu|(t, \omega)\{(A-t) \cap[-r, 0]\}
$$

for all Borel subsets $A$ of $[-r, \infty)$, where $|\nu|$ is the total variation measure of $\nu$ with respect to the Euclidean norm on $\mathbf{R}^{d \times d}$. Therefore the equation

$$
\mu(\omega)(\cdot):=\int_{0}^{\infty} \bar{\nu}(t, \omega)(\cdot) d t
$$

defines a positive measure on $[-r, \infty)$. For each $\omega \in \Omega$ suppose that $\mu(\omega)$ has a density with respect to Lebesgue measure, and the density is locally essentially bounded. (Note that this condition is automatically satisfied if $\nu(t, \omega)$ is independent of $(t, \omega)$.)

(ii) $K: \mathbf{R} \times \Omega \rightarrow L^{\infty}\left([-r, 0], \mathbf{R}^{d \times d}\right)$ is measurable and $\left(\mathcal{F}_{t}\right)_{t \geq 0^{-}}$adapted. Define the random field $\bar{K}(t, s, \omega)$ by $\bar{K}(t, s, \omega):=K(t, \omega)(s-t)$ for $t \geq 0,-r \leq s-t \leq 0$. Assume that $\bar{K}(t, s, \omega)$ is absolutely continuous in $t$ for Lebesgue a.a. $s \in[-r, 0]$ and all $\omega \in \Omega$. For every $\omega \in \Omega, \frac{\partial \bar{K}}{\partial t}(t, s, \omega)$ and $\bar{K}(t, s, \omega)$ are locally essentially bounded in $(t, s)$. $\frac{\partial \bar{K}}{\partial t}(t, s, \omega)$ is jointly measurable.

(iii) $L=M+V$, where $M$ is a continuos $\mathbf{R}^{d \times d}$-valued, $\left(\mathcal{F}_{t}\right)_{t \geq 0}$-local martingale, and $V$ is an $\mathbf{R}^{d \times d}$-valued, $\left(\mathcal{F}_{t}\right)_{t \geq 0}$-adapted bounded-variation process. The process $N$ is

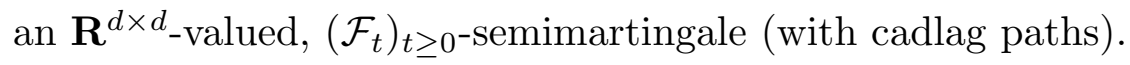

Theorem III.5. ([MS1])

Under hypotheses ( $R)$, equation (IX) is regular with respect to $M_{2}$ with a measurable flow $X: \mathbf{R}^{+} \times M_{2} \times \Omega \rightarrow M_{2}$. This flow satisfies the conclusions of Theorem III.4. 
Proof.

The key idea of the proof is to use integration by parts ([Met], pp. 185) in order to identify the sfde (IX) with the following random family of linear integral equations:

$$
\begin{aligned}
x(t) & =\phi(t, \omega)\left[v-\int_{0}^{t} Z(s, \omega) \bar{K}(s, s, \omega) x(s) d s+\int_{-r}^{t-r} Z(s+r, \omega) \bar{K}(s+r, s, \omega) x(s) d s\right. \\
& -\int_{-r}^{t} \int_{s \vee 0}^{t \wedge(s+r)} Z(u, \omega) \frac{\partial}{\partial u} \bar{K}(u, s, \omega) d u x(s) d s+Z(t, \omega) \int_{t-r}^{t} \bar{K}(t, s, \omega) x(s) d s \\
& +\int_{-r}^{t} \int_{[(s-t) \vee(-r), 0 \wedge s]} \phi^{-1}(s-u, \omega) \nu(s-u, \omega)(d u) x(s) d s \\
& \left.+\int_{0}^{t} \phi^{-1}(s, \omega) d V(s, \omega) x(s-)-\int_{-r}^{t} \int_{s \vee 0}^{t \wedge(s+r)} \phi^{-1}(u, \omega) d[M, N](u, \omega) \bar{K}(u, s, \omega) x(s) d s\right],
\end{aligned}
$$

for $t \in \mathbf{R}^{+}$, with the initial condition

$$
\left(x(0), x_{0}\right)=(v, \eta) \in M_{2} .
$$

The processes $\phi$ and $Z$ in the above integral equation are defined by

$$
\begin{aligned}
d \phi(t) & =d M(t) \phi(t), \quad t>0 \\
\phi(0) & =I \in \mathbf{R}^{d \times d}
\end{aligned}
$$

and

$$
Z(t):=\int_{0}^{t} \phi^{-1}(u) d N(u), \quad t \geq 0
$$

The crucial point is that the above integral equation contains no stochastic integrals. A somewhat lengthy pathwise analysis of the random integral equation yields the existence and regularity of the semiflow ([MS1], pp. 85-96).

\section{Regular non-linear systems.}

Let $\left(\Omega, \mathcal{F},\left(\mathcal{F}_{t}\right)_{t \geq 0}, P\right)$ be the complete filtered Wiener space described in Section 3 . Consider the following non-linear sfde with ordinary diffusion coefficients:

$$
\left.\begin{array}{rl}
d x(t) & =H\left(x_{t}\right) d t+\sum_{i=1}^{m} g_{i}(x(t)) d W_{i}(t), \quad t>0 \\
x_{0} & =\eta \in C .
\end{array}\right\}
$$

Here, $H: C \rightarrow \mathbf{R}^{d}$ is globally Lipschitz, $g_{i}: \mathbf{R}^{d} \rightarrow \mathbf{R}^{d}$ are $C^{2}$-bounded maps satisfying the Frobenius condition (vanishing Lie brackets):

$$
D g_{i}(v) g_{j}(v)=D g_{j}(v) g_{i}(v), \quad 1 \leq i, j \leq m, v \in \mathbf{R}^{d}
$$

and $W:=\left(W_{1}, W_{2}, \cdots, W_{m}\right)$ is $m$-dimensional Brownian motion. Note that the diffusion coefficient in $(\mathrm{XV})$ has no memory. 
Theorem III.6. ([M1])

Suppose the above conditions hold. Then the trajectory field $\left\{{ }^{\eta} x_{t}: t \geq 0, \eta \in C\right\}$ of $(X V)$ has a measurable version $X: \mathbf{R}^{+} \times C \times \Omega \rightarrow C$ satisfying the following properties:

For each $\alpha \in(0,1 / 2)$, there is a set $\Omega_{\alpha} \subset \Omega$ of full Wiener measure such that for every $\omega \in \Omega_{\alpha}$ the following is true:

(i) $X(\cdot, \cdot, \omega): \mathbf{R}^{+} \times C \rightarrow C$ is continuous;

(ii) $X(\cdot, \cdot, \omega):[r, \infty) \times C \rightarrow C^{\alpha}$ is continuous;

(iii) for each $t \geq r, X(t, \cdot, \omega): C \rightarrow C$ is compact;

(iv) for each $t \geq r, X(t, \cdot, \omega): C \rightarrow C^{\alpha}$ is Lipschitz on every bounded set in $C$, with a Lipschitz constant independent of $t$ in compact sets. Hence, each map $X(t, \cdot, \omega)$ : $C \rightarrow C$ is compact; viz. it takes bounded sets into relatively compact sets.

Proof of Theorem III.6.

We use a non-linear variational method originally due to Sussman ([Su]) and Doss ([Do]) in the nondelay case $r=0$. See ([M1], Theorem (2.1), Chapter (V), p. 121).

Write $g:=\left(g_{1}, g_{2}, \cdots, g_{m}\right): \mathbf{R}^{d} \rightarrow \mathbf{R}^{d \times m}$. By the Frobenius condition, there is a $C^{2}$ map $F: \mathbf{R}^{m} \times \mathbf{R}^{d} \rightarrow \mathbf{R}^{d}$ such that $\left\{F(\underline{t}, \cdot): \underline{t} \in \mathbf{R}^{m}\right\}$ is a group of $C^{2}$ diffeomorphisms of $\mathbf{R}^{d}$ satisfying

$$
\begin{aligned}
D_{1} F(\underline{t}, x) & =g(F(\underline{t}, x)), \\
F(\underline{0}, x) & =x
\end{aligned}
$$

for all $\underline{t} \in \mathbf{R}^{m}, x \in \mathbf{R}^{d}$.

Define

$$
W^{0}(t):=\left\{\begin{array}{l}
W(t)-W(0), \quad t \geq 0, \\
0 \quad-r \leq t<0
\end{array}\right.
$$

and $\tilde{H}: \mathbf{R}^{+} \times C \times \Omega \rightarrow \mathbf{R}^{d}$, by

$$
\begin{aligned}
\tilde{H}(t, \eta, \cdot):=\left[D_{2} F(\right. & \left.\left.W^{0}(t), \eta(0)\right)\right]^{-1}\left\{H\left[F \circ\left(W_{t}^{0}, \eta\right)\right]\right. \\
& \left.-\frac{1}{2} \operatorname{trace}\left(D g\left[F\left(W^{0}(t), \eta(0)\right)\right] \circ g\left[F\left(W^{0}(t), \eta(0)\right)\right]\right)\right\},
\end{aligned}
$$

where the expression under the "trace" is viewed as a bilinear form $\mathbf{R}^{m} \times \mathbf{R}^{m} \rightarrow \mathbf{R}^{d}$, and the trace has values in $\mathbf{R}^{d}$. By the hypotheses on $H$ and $g$, it follows that for each $\omega$, $\tilde{H}(t, \eta, \omega)$ is jointly continuous in $(t, \eta)$, Lipschitz in $\eta$ in bounded subsets of $C$ uniformly for $t$ in compact sets, and satisfies a global linear growth condition in $\eta$ ([M1], pp. 114-126). 
For each $\omega \in \Omega$, let ${ }^{\eta} \xi(\cdot, \omega)$ denote the unique solution of the random fde

$$
\begin{aligned}
\eta^{\eta} \xi^{\prime}(t, \omega) & =\tilde{H}\left(t,{ }^{\eta} \xi_{t}, \omega\right), \quad t>0, \\
\eta^{\eta} \xi_{0}(\omega) & =\eta .
\end{aligned}
$$

Define the semiflow

$$
X(t, \eta, \omega):=F \circ\left(W_{t}^{0}(\omega),{ }^{\eta} \xi_{t}(\omega)\right)
$$

for $t \geq 0, \eta \in C, \omega \in \Omega$. Therefore $X$ satisfies all assertions of the theorem ([M1], pp. 126-133).

\section{Remark.}

The issue of regularity for a much more general class of non-linear sfde's will be addressed elsewhere in joint work of M. Scheutzow and the author. 


\section{Chapter IV}

\section{Ergodic Theory of Linear SFDE's}

In this chapter, we shall study more closely the regular linear sfde's (VIII) and (IX) of Chapter III. Our approach is to view the sfde's (VIII) and (IX) as (almost sure) stochastic dynamical systems on the infinite-dimensional state space $M_{2}$. In particular, we will establish the following:

(i) Existence of a "perfect" cocycle on $M_{2}$ that is a modification of the trajectory field $\left(x(t), x_{t}\right) \in M_{2}$.

(ii) Existence of almost sure Lyapunov exponents $\lim _{t \rightarrow \infty} \frac{1}{t} \log \left\|\left(x(t), x_{t}\right)\right\|_{M_{2}}$.

(iii) The multiplicative ergodic theorem and the concept of hyperbolicity of the cocycle.

(iv) The Stable Manifold Theorem, (viz. the existence of "random saddles") for regular hyperbolic linear sfde's.

\section{Regular linear systems driven by white noise.}

Recall the class of regular linear sfde's

$$
\left.\begin{array}{rl}
d x(t) & =H\left(x\left(t-d_{1}\right), \cdots, x\left(t-d_{N}\right), x(t), x_{t}\right) d t+\sum_{i=1}^{m} g_{i} x(t) d W_{i}(t), \quad t>0 \\
\left(x(0), x_{0}\right) & =(v, \eta) \in M_{2}
\end{array}\right\}
$$

which was introduced in Section 3 of Chapter III. We will adopt the basic setting therein. In particular, we will denote by $X: \mathbf{R}^{+} \times M_{2} \times \Omega \rightarrow M_{2}$ the semiflow for (VIII) given by Theorem III.4. The compactness of $X(t, \cdot, \omega)$, for $t \geq r$, will be used below to construct the Lyapunov spectrum, define hyperbolicity for (VIII), and establish the associated exponential dichotomies.

Define the canonical Brownian shift $\theta: \mathbf{R} \times \Omega \rightarrow \Omega$ on Wiener space by

$$
\theta(t, \omega)(u):=\omega(t+u)-\omega(t), \quad u, t \in \mathbf{R}, \quad \omega \in \Omega .
$$

We will first show that the version $X$ of the flow constructed in Theorem III.4 is a multiplicative $L\left(M_{2}\right)$-valued linear cocycle over $\theta$. Indeed we have 
Theorem IV.1. ([M3]) (The Cocycle Property)

There is an $\mathcal{F}$-measurable set $\hat{\Omega}$ of full P-measure such that $\theta(t, \cdot)(\hat{\Omega}) \subseteq \hat{\Omega}$ for all $t \geq 0$ and

$$
X\left(t_{2}, \cdot, \theta\left(t_{1}, \omega\right)\right) \circ X\left(t_{1}, \cdot, \omega\right)=X\left(t_{1}+t_{2}, \cdot, \omega\right)
$$

for all $\omega \in \hat{\Omega}$ and all $t_{1}, t_{2} \geq 0$.

The figure below illustrates the cocycle property. The vertical solid lines represent random fibers consisting of copies of the state space $M_{2}$. The pair $(X, \theta)$ is a random vector-bundle morphism over the base probability space $\Omega$.

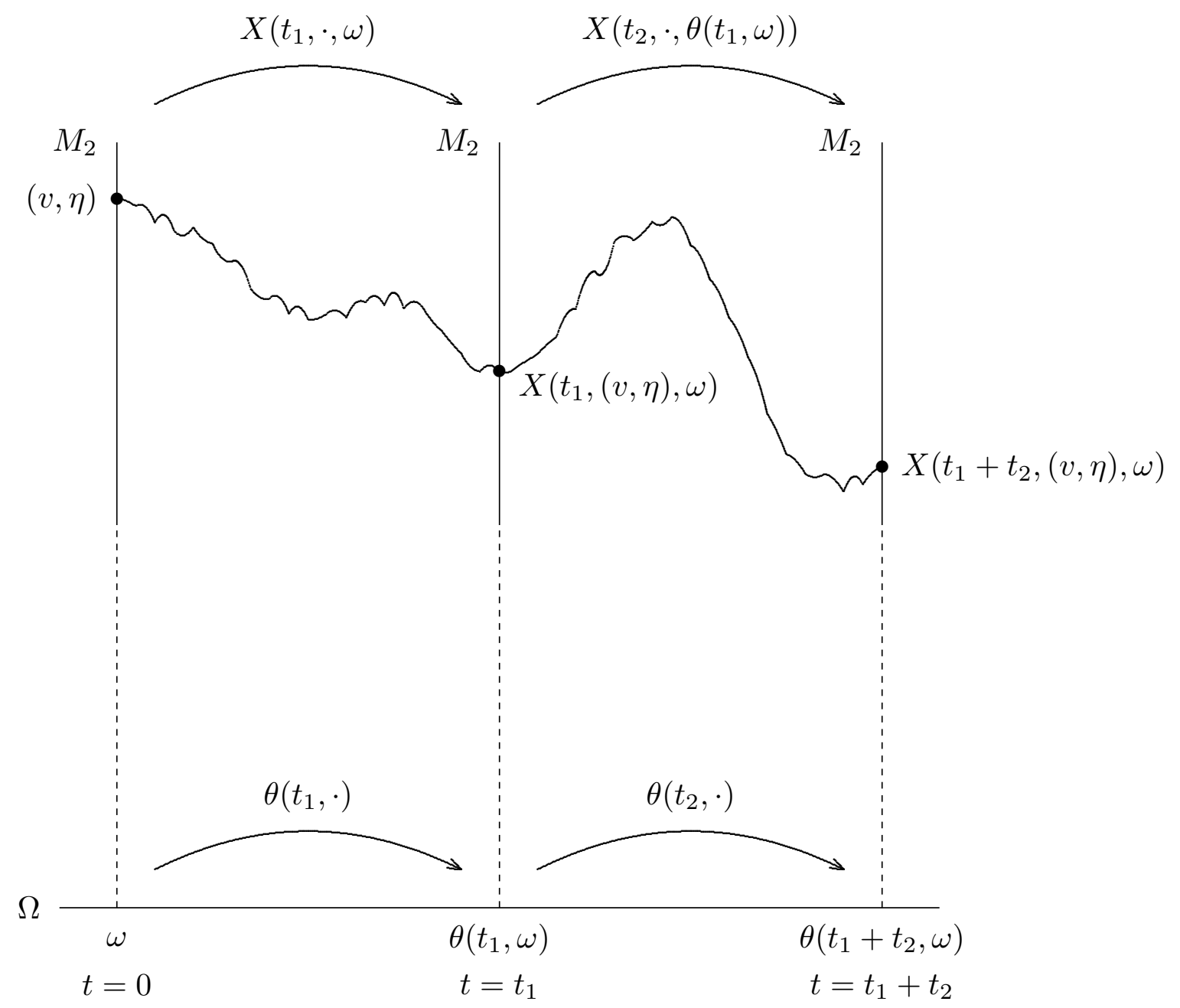

Note that the family $\tilde{X}_{t}: M_{2} \times \Omega \rightarrow M_{2} \times \Omega, t \geq 0$,

$$
\tilde{X}_{t}((v, \eta), \omega):=(X(t,(v, \eta), \omega), \theta(t, \omega)), \quad(v, \eta) \in M_{2}, \omega \in \Omega
$$


is a one-parameter semigroup of morphisms on the trivial bundle $M_{2} \times \Omega$.

We now sketch a proof of Theorem IV.1.

Proof of Theorem IV.1.

For simplicity consider the case of a single delay $d_{1}$; i.e. $N=1$ in (VIII). We will break the proof up into three steps.

First step.

Approximate the Brownian motion $W$ in (VIII) by smooth $\left(\mathcal{F}_{t}\right)_{t \geq 0}$-adapted processes $\left\{W^{k}\right\}_{k=1}^{\infty}$, where

$$
W^{k}(t):=k \int_{t-(1 / k)}^{t} W(u) d u-k \int_{-(1 / k)}^{0} W(u) d u, \quad t \geq 0, k \geq 1 .
$$

It is easy to check that each $W^{k}$ is a helix in the sense that

$$
W^{k}\left(t_{1}+t_{2}, \omega\right)-W^{k}\left(t_{1}, \omega\right)=W^{k}\left(t_{2}, \theta\left(t_{1}, \omega\right)\right), \quad t_{1}, t_{2} \in \mathbf{R}, \omega \in \Omega .
$$

(The above helix property implies that each $W^{k}$ has stationary increments.)

Let $X^{k}: \mathbf{R}^{+} \times M_{2} \times \Omega \rightarrow M_{2}$ be the stochastic semiflow of the random fde's:

$$
\left.\begin{array}{rl}
d x^{k}(t) & =H\left(x^{k}\left(t-d_{1}\right), x^{k}(t), x_{t}^{k}\right) d t+\sum_{i=1}^{m} g_{i} x(t)\left(W_{i}^{k}\right)^{\prime}(t) d t-\frac{1}{2} \sum_{i=1}^{m} g_{i}^{2} x^{k}(t) d t, \quad t>0 \\
\left(x^{k}(0), x_{0}^{k}\right) & =(v, \eta) \in M_{2}, \quad k \geq 1 .
\end{array}\right\}
$$

If $X: \mathbf{R}^{+} \times M_{2} \times \Omega \rightarrow M_{2}$ is the version of the trajectory field of (VIII) given in Theorem III.4, then there exists an $\mathcal{F}$-measurable set $\hat{\Omega}$ of full Wiener measure which is invariant under $\theta(t, \cdot)$ for all $t \geq 0$ and is such that

$$
\lim _{k \rightarrow \infty} \sup _{0 \leq t \leq T}\left\|X^{k}(t, \cdot, \omega)-X(t, \cdot, \omega)\right\|_{L\left(M_{2}\right)}=0
$$

for every $0<T<\infty$ and all $\omega \in \hat{\Omega}$. The convergence in (3) may be proved using the following stochastic variational method.

Let $\phi: \mathbf{R}^{+} \times \Omega \rightarrow \mathbf{R}^{d \times d}$ be the $d \times d$-matrix-valued solution of the linear Itô sode (without delay):

$$
\left.\begin{array}{rl}
d \phi(t) & =\sum_{i=1}^{m} g_{i} \phi(t) d W_{i}(t), \quad t>0 \\
\phi(0) & =I \in \mathbf{R}^{d \times d} .
\end{array}\right\}
$$


Denote by $\phi^{k}: \mathbf{R}^{+} \times \Omega \rightarrow \mathbf{R}^{d \times d}, k \geq 1$, the $d \times d$-matrix solution of the random family of linear ode's:

$$
\left.\begin{array}{rl}
d \phi^{k}(t) & =\sum_{i=1}^{m} g_{i} \phi^{k}(t)\left(W_{i}^{k}\right)^{\prime}(t)-\frac{1}{2} \sum_{i=1}^{m} g_{i}^{2} \phi^{k}(t) d t, \quad t>0 \\
\phi^{k}(0) & =I \in \mathbf{R}^{d \times d} .
\end{array}\right\}
$$

Let $\hat{\Omega}$ be the sure event consisting of all $\omega \in \Omega$ such that

$$
\phi(t, \omega):=\lim _{k \rightarrow \infty} \phi^{k}(t, \omega)
$$

exists uniformly for $t$ in compact subsets of $\mathbf{R}^{+}$. Each $\phi^{k}$ is an $\mathbf{R}^{d \times d}$-valued cocycle over $\theta$, viz.

$$
\phi^{k}\left(t_{1}+t_{2}, \omega\right)=\phi^{k}\left(t_{2}, \theta\left(t_{1}, \omega\right)\right) \phi^{k}\left(t_{1}, \omega\right)
$$

for all $t_{1}, t_{2} \in \mathbf{R}^{+}$and $\omega \in \Omega$. Using the definition of $\hat{\Omega}$ and passing to the limit in (6) as $k \rightarrow \infty$, we conclude that $\{\phi(t, \omega): t \geq 0, \omega \in \Omega\}$ is an $\mathbf{R}^{d \times d}$-valued perfect cocycle over $\theta$, viz.

(i) $P(\hat{\Omega})=1$;

(ii) $\theta(t, \cdot)(\hat{\Omega}) \subseteq \hat{\Omega}$ for all $t \geq 0$;

(iii) $\phi\left(t_{1}+t_{2}, \omega\right)=\phi\left(t_{2}, \theta\left(t_{1}, \omega\right)\right) \phi\left(t_{1}, \omega\right)$ for all $t_{1}, t_{2} \geq 0$ and every $\omega \in \hat{\Omega}$;

(iv) $\phi(\cdot, \omega)$ is continuous for every $\omega \in \hat{\Omega}$.

An alternative approach is to use the "perfection theorem" in ([MS1], Theorem 3.1, p. 79-82) for crude cocycles with values in a metrizable second countable topological group. (Observe that $\phi(t, \omega) \in G L\left(\mathbf{R}^{d}\right)$ a.s.)

Let $J:=[-r, 0]$. Define $\hat{H}: \mathbf{R}^{+} \times \mathbf{R}^{d} \times M_{2} \times \Omega \rightarrow \mathbf{R}^{d}$ by

$$
\hat{H}\left(t, v_{1}, v, \eta, \omega\right):=\phi(t, \omega)^{-1}\left[H\left(\phi_{t}(\cdot, \omega)\left(-d_{1}, v_{1}\right), \phi(t, \omega)(v), \phi_{t}(\cdot, \omega) \circ\left(i d_{J}, \eta\right)\right)\right]
$$

for $\omega \in \Omega, t \geq 0, v, v_{1} \in \mathbf{R}^{d}, \eta \in L^{2}\left([-r, 0], \mathbf{R}^{d}\right)$, where

$$
\phi_{t}(\cdot, \omega)(s, v)= \begin{cases}\phi(t+s, \omega)(v), & t+s \geq 0 \\ v, & -r \leq t+s<0\end{cases}
$$

and

$$
\left(i d_{J}, \eta\right)(s)=(s, \eta(s)), \quad s \in J
$$


Define $\hat{H}^{k}: \mathbf{R}^{+} \times \mathbf{R}^{d} \times M_{2} \times \Omega \rightarrow \mathbf{R}^{d}$ by a relation similar to (7) with $\phi$ replaced by $\phi^{k}$. Then the random fde's

$$
\left.\begin{array}{rl}
y^{\prime}(t) & =\hat{H}\left(t, y\left(t-d_{1}\right), y(t), y_{t}, \omega\right), \quad t>0 \\
\left.y(0), y_{0}\right) & =(v, \eta) \in M_{2}, \\
y^{k^{\prime}}(t) & =\hat{H}^{k}\left(t, y^{k}\left(t-d_{1}\right), y^{k}(t), y_{t}^{k}, \omega\right), \quad t>0 \\
\left.(0), y_{0}^{k}\right) & =(v, \eta) \in M_{2},
\end{array}\right\}
$$

have unique non-explosive solutions $y, y^{k}:[-r, \infty) \times \Omega \rightarrow \mathbf{R}^{d}$ ([M3], pp. 93-98). Itô's formula implies that

$$
X(t,(v, \eta), \omega)=\left(\phi(t, \omega)(y(t, \omega)), \phi_{t}(\cdot, \omega) \circ\left(i d_{J}, y_{t}\right)\right) .
$$

The chain rule gives a similar relation for $X^{k}$ with $\phi$ replaced by $\phi^{k}$ ([M3], pp. 96-97).

Using (5) and the definitions of $\hat{H}$ and $\hat{H}^{k}$, we get the convergence

$$
\lim _{k \rightarrow \infty}\left|\hat{H}^{k}\left(t, v_{1}, v, \eta, \omega\right)-\hat{H}\left(t, v_{1}, v, \eta, \omega\right)\right|=0
$$

for every $\omega \in \hat{\Omega}$, uniformly for $\left(t, v_{1}, v, \eta\right)$ in bounded sets of $\mathbf{R}^{+} \times \mathbf{R}^{d} \times M_{2}$. Gronwall's lemma and (11) give (3).

\section{Second step.}

Fix $\omega \in \hat{\Omega}$, use uniqueness of solutions to the approximating equation (VIII-k) and the helix property (2) of $W^{k}$ to obtain the following cocycle property for $\left(X^{k}, \theta\right)$ :

$$
X^{k}\left(t_{2}, \cdot, \theta\left(t_{1}, \omega\right)\right) \circ X^{k}\left(t_{1}, \cdot, \omega\right)=X^{k}\left(t_{1}+t_{2}, \cdot, \omega\right)
$$

for all $\omega \in \hat{\Omega}$ and $t_{1}, t_{2} \geq 0, k \geq 1$.

Third step.

Pass to limit as $k \rightarrow \infty$ in the above identity, and use the convergence (3) in the uniform operator norm to get the required perfect cocycle property for $X$.

The a.s. Lyapunov exponents

$$
\lim _{t \rightarrow \infty} \frac{1}{t} \log \|X(t,(v(\omega), \eta(\omega)), \omega)\|_{M_{2}},
$$

(for a.a. $\omega \in \Omega,(v, \eta) \in L^{2}\left(\Omega, M_{2}\right)$ ) of the sfde (VIII) are characterized by the following "spectral theorem". The proof of Theorem IV.2 uses compactness of $X(t, \cdot, \omega): M_{2} \rightarrow M_{2}$, $t \geq r$, together with Ruelle-Oseledec infinite-dimensional multiplicative ergodic theorem $([\mathrm{R}])$.

Note that each shift $\theta(t, \cdot)$ is ergodic and preserves Wiener measure $P$. 
Theorem IV.2. ([M3])(Spectral Theorem)

Let $X: \mathbf{R}^{+} \times M_{2} \times \Omega \rightarrow M_{2}$ be the flow of (VIII) given in Theorem III.4. Then there exist

(a) an $\mathcal{F}$-measurable set $\Omega^{*} \subseteq \Omega$ such that $P\left(\Omega^{*}\right)=1$ and $\theta(t, \cdot)\left(\Omega^{*}\right) \subseteq \Omega^{*}$ for all $t \geq 0$,

(b) a fixed (non-random) sequence of real numbers $\left\{\lambda_{i}\right\}_{i=1}^{\infty}$, and

(c) a random family $\left\{E_{i}(\omega): i \geq 1, \omega \in \Omega^{*}\right\}$ of (closed) finite-codimensional subspaces of $M_{2}$, with the following properties:

(i) If the Lyapunov spectrum $\left\{\lambda_{i}\right\}_{i=1}^{\infty}$ is infinite, then $\lambda_{i+1}<\lambda_{i}$ for all $i \geq 1$ and $\lim _{i \rightarrow \infty} \lambda_{i}=-\infty$; otherwise there is a fixed (non-random) integer $N_{0} \geq 1$ such that $\lambda_{N_{0}}=-\infty<\lambda_{N_{0}-1}<\cdots<\lambda_{2}<\lambda_{1}$;

(ii) each map $\omega \mapsto E_{i}(\omega), i \geq 1$, is $\mathcal{F}$-measurable into the Grassmannian of $M_{2}$;

(iii) $E_{i+1}(\omega) \subset E_{i}(\omega) \subset \cdots \subset E_{2}(\omega) \subset E_{1}(\omega)=M_{2}, i \geq 1, \omega \in \Omega^{*}$;

(iv) for each $i \geq 1$, codim $E_{i}(\omega)$ is fixed independently of $\omega \in \Omega^{*}$;

(v) for each $\omega \in \Omega^{*}$ and $(v, \eta) \in E_{i}(\omega) \backslash E_{i+1}(\omega)$,

$$
\lim _{t \rightarrow \infty} \frac{1}{t} \log \|X(t,(v, \eta), \omega)\|_{M_{2}}=\lambda_{i}, i \geq 1
$$

(vi) top exponent:

$$
\lambda_{1}=\lim _{t \rightarrow \infty} \frac{1}{t} \log \|X(t, \cdot, \omega)\|_{L\left(M_{2}\right)} \quad \text { for all } \omega \in \Omega^{*}
$$

(vii) invariance:

$$
X(t, \cdot, \omega)\left(E_{i}(\omega)\right) \subseteq E_{i}(\theta(t, \omega))
$$

for all $\omega \in \Omega^{*}, t \geq 0, i \geq 1$.

In the figure below, each $E_{i}$ corresponds to a random subbundle of the trivial bundle $M_{2} \times \Omega$, which is invariant under the morphism $(X, \theta)$. 


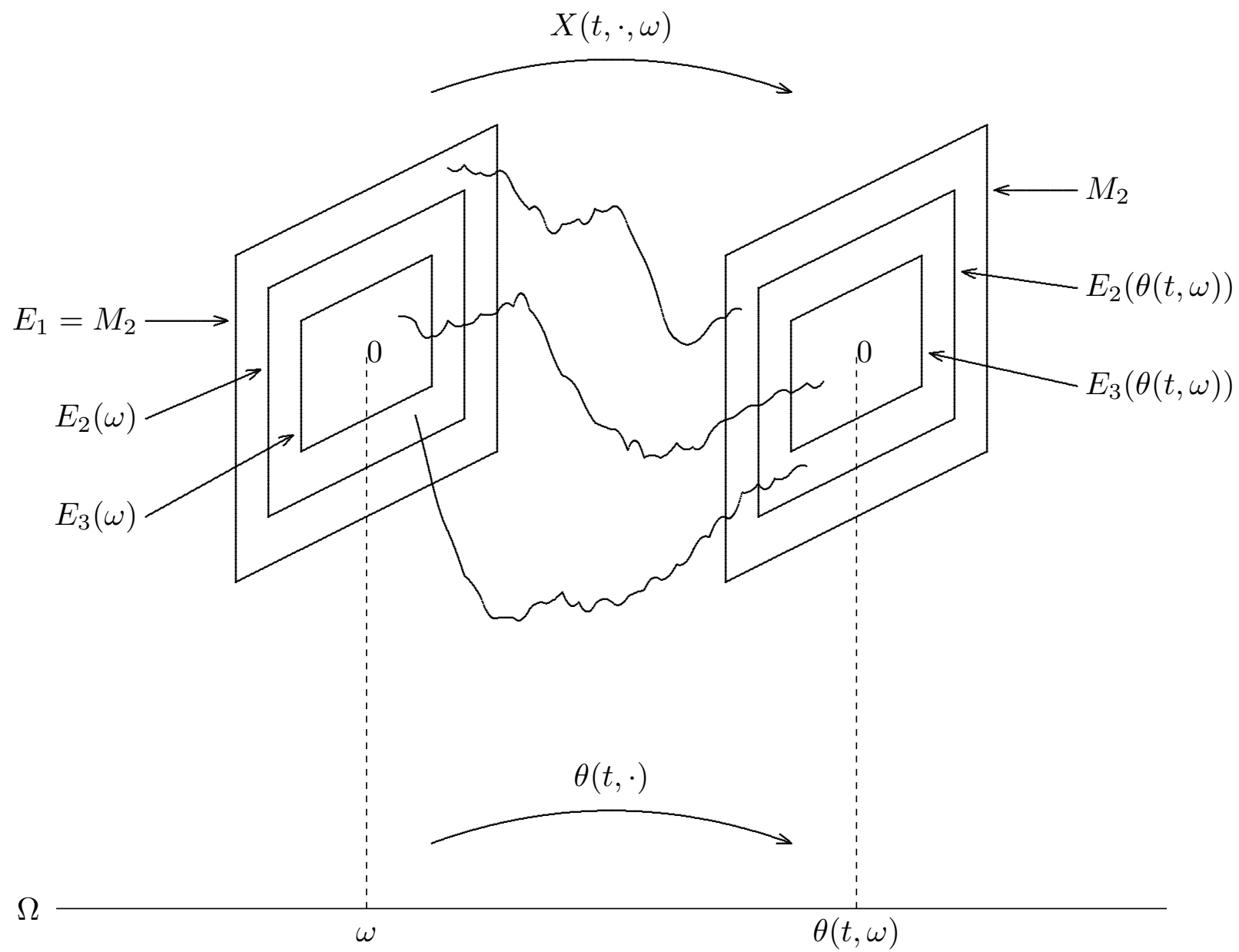

The proof of Theorem IV.2 is based on a discrete version of Oseledec's multiplicative ergodic theorem in Hilbert space, developed by Ruelle ([R], Theorem (1.1), p. 248 and Corollary (2.2), p. 253). For any real-valued function $f$, denote by $f^{+}:=f \vee 0$ its positive part.

Theorem IV.3. $([R])$

Let $(\Omega, \mathcal{F}, P)$ be a probability space and $\tau: \Omega \rightarrow \Omega$ a P-preserving transformation. Assume that $H$ is a separable Hilbert space and $T: \Omega \rightarrow L(H)$ a measurable map (with respect to the Borel field on the space of all bounded linear operators $L(H))$. Suppose that $T(\omega)$ is compact for almost all $\omega \in \Omega$, and $E \log ^{+}\|T(\cdot)\|_{L(H)}<\infty$. Define the family $\left\{T^{n}(\omega): \omega \in \Omega, n \geq 1\right\}$ of compact linear operators by

$$
T^{n}(\omega):=T\left(\tau^{n-1}(\omega)\right) \circ \cdots T(\tau(\omega)) \circ T(\omega)
$$


for $\omega \in \Omega, n \geq 1$.

Then there is a set $\Omega_{0} \in \mathcal{F}$ of full P-measure such that $\tau\left(\Omega_{0}\right) \subseteq \Omega_{0}$, and for each $\omega \in \Omega_{0}$, the limit

$$
\Lambda(\omega):=\lim _{n \rightarrow \infty}\left[T^{n}(\omega)^{*} \circ T^{n}(\omega)\right]^{1 /(2 n)}
$$

exists in the uniform operator norm and is a positive compact self-adjoint operator on $H$. Furthermore, each $\Lambda(\omega)$ has a discrete spectrum

$$
e^{\mu_{1}(\omega)}>e^{\mu_{2}(\omega)}>e^{\mu_{3}(\omega)}>e^{\mu_{4}(\omega)}>\cdots
$$

where the $\mu_{i}$ 's are distinct. If the sequence $\left\{\mu_{i}\right\}_{i=1}^{\infty}$ is infinite, then $\mu_{i} \downarrow-\infty$; otherwise it terminates at $\mu_{N_{0}(\omega)}=-\infty$. If $\mu_{i}(\omega)>-\infty$, then $e^{\mu_{i}(\omega)}$ has finite multiplicity $m_{i}(\omega)$ and a finite-dimensional eigen-space $F_{i}(\omega)$, with $m_{i}(\omega):=\operatorname{dim} F_{i}(\omega)$. Define

$$
E_{1}(\omega):=M_{2}, \quad E_{i}(\omega):=\left[\oplus_{j=1}^{i-1} F_{j}(\omega)\right]^{\perp}, \quad E_{\infty}(\omega):=\operatorname{ker} \Lambda(\omega)
$$

Then

$$
E_{\infty}(\omega) \subset \cdots \subset E_{i+1}(\omega) \subset E_{i}(\omega) \cdots \subset E_{2}(\omega) \subset E_{1}(\omega)=H
$$

and

$$
\lim _{n \rightarrow \infty} \frac{1}{n} \log \left\|T^{n}(\omega) x\right\|_{H}=\left\{\begin{array}{l}
\mu_{i}(\omega), \quad \text { if } \quad x \in E_{i}(\omega) \backslash E_{i+1}(\omega) \\
-\infty, \quad \text { if } \quad x \in \operatorname{ker} \Lambda(\omega)
\end{array}\right.
$$

For a proof of Theorem IV.3 see [R], pp. 248-254.

The following "perfect" version of Kingman's subadditive ergodic theorem is also used to construct the shift invariant set $\Omega^{*}$ appearing in Theorem IV.2 above.

Theorem IV.4. ([M3])("Perfect" Subadditive Ergodic Theorem)

Let $f: \mathbf{R}^{+} \times \Omega \rightarrow \mathbf{R} \cup\{-\infty\}$ be a measurable process on a complete probability space $(\Omega, \mathcal{F}, P)$ and $\tau(t, \cdot): \Omega \rightarrow \Omega, t \geq 0$, a family of $P$-preserving transformations such that

(i) $E \sup _{0 \leq u \leq 1} f^{+}(u, \cdot)<\infty, E \sup _{0 \leq u \leq 1} f^{+}(1-u, \tau(u, \cdot))<\infty$;

(ii) $f\left(t_{1}+t_{2}, \omega\right) \leq f\left(t_{1}, \omega\right)+f\left(t_{2}, \tau\left(t_{1}, \omega\right)\right)$ for all $t_{1}, t_{2} \geq 0$ and every $\omega \in \Omega$.

Then there exist a set $\hat{\hat{\Omega}} \in \mathcal{F}$ and a measurable $\tilde{f}: \Omega \rightarrow \mathbf{R} \cup\{-\infty\}$ with the properties:

(a) $P(\hat{\hat{\Omega}})=1, \tau(t, \cdot)(\hat{\hat{\Omega}}) \subseteq \hat{\hat{\Omega}}$ for all $t \geq 0$;

(b) $\tilde{f}(\omega)=\tilde{f}(\tau(t, \omega))$ for all $\omega \in \hat{\hat{\Omega}}$ and all $t \geq 0$;

(c) $\tilde{f}^{+} \in \mathbf{L}^{1}(\Omega, \mathbf{R} ; P)$; 


$$
\text { (d) } \lim _{t \rightarrow \infty}(1 / t) f(t, \omega)=\tilde{f}(\omega) \text { for every } \omega \in \hat{\hat{\Omega}} \text {. }
$$

If $\tau(t, \cdot)$ is ergodic, then there exist $f^{*} \in \mathbf{R} \cup\{-\infty\}$ and $\tilde{\tilde{\Omega}} \in \mathcal{F}$ such that

$$
\begin{aligned}
& (a)^{\prime} P(\tilde{\tilde{\Omega}})=1, \tau(t, \cdot)(\tilde{\tilde{\Omega}}) \subseteq \tilde{\tilde{\Omega}}, t \geq 0 ; \\
& (b)^{\prime} \tilde{f}(\omega)=f^{*}=\lim _{t \rightarrow \infty}(1 / t) f(t, \omega) \text { for every } \omega \in \tilde{\tilde{\Omega}} .
\end{aligned}
$$

The proof of Theorem IV.4 is given in [M3], Lemma 7, pp. 115-117. The proof of Theorem IV.2 is an application of Theorem IV.3. It requires Theorem IV.4 and the following sequence of lemmas.

\section{Lemma IV.1.}

For each integer $k \geq 1$ and any $0<a<\infty$,

$$
\begin{gathered}
E \sup _{0 \leq t \leq a}\left\|\phi(t, \omega)^{-1}\right\|^{2 k}<\infty ; \\
E \sup _{0 \leq t_{1}, t_{2} \leq a}\left\|\phi\left(t_{2}, \theta\left(t_{1}, \cdot\right)\right)\right\|^{2 k}<\infty .
\end{gathered}
$$

Proof.

Observe that $\hat{\phi}(t, \omega):=\phi(t, \omega)^{-1}$ is a solution of a linear sde in $L\left(\mathbf{R}^{d}\right)$. Therefore it is a semimartingale. The conclusions of the lemma then follow from standard sode estimates, the cocycle property for $(\phi, \theta)$, and Hölder's inequality ([M3], pp. 106-108).

The next lemma is the crucial estimate needed to apply the Ruelle-Oseledec theorem (Theorem IV.3).

\section{Lemma IV.2.}

$$
E \sup _{0 \leq t_{1}, t_{2} \leq r} \log ^{+}\left\|X\left(t_{2}, \cdot, \theta\left(t_{1}, \cdot\right)\right)\right\|_{L\left(M_{2}\right)}<\infty
$$

\section{Proof.}

If $y(t,(v, \eta), \omega)$ is the solution of the fde (8), then using Gronwall's inequality, taking " $E \sup _{0 \leq t_{1}, t_{2} \leq r} \log ^{+} \sup _{\|(v, \eta)\| \leq 1}$ " and applying Lemma IV.1, gives

$$
E \sup _{0 \leq t_{1}, t_{2} \leq r} \log ^{+} \sup _{\|(v, \eta)\| \leq 1}\left\|\left(y\left(t_{2},(v, \eta), \theta\left(t_{1}, \cdot\right)\right), y_{t_{2}}\left(\cdot,(v, \eta), \theta\left(t_{1}, \cdot\right)\right)\right)\right\|_{M_{2}}<\infty .
$$


The conclusion of the lemma now follows from the above result by replacing $\omega^{\prime}$ with $\theta\left(t_{1}, \omega\right)$ in the formula

$$
X\left(t_{2},(v, \eta), \omega^{\prime}\right)=\left(\phi\left(t_{2}, \omega^{\prime}\right)\left(y\left(t_{2},(v, \eta), \omega^{\prime}\right)\right), \phi_{t_{2}}\left(\cdot, \omega^{\prime}\right) \circ\left(i d_{J}, y_{t_{2}}\left(\cdot,(v, \eta), \omega^{\prime}\right)\right)\right.
$$

and applying Lemma IV.1.

The existence of the Lyapunov spectrum is obtained by interpolating between delay periods of length $r$, using the discrete limit

$$
\frac{1}{r} \lim _{k \rightarrow \infty} \frac{1}{k} \log \|X(k r,(v(\omega), \eta(\omega)), \omega)\|_{M_{2}},
$$

a.a. $\omega \in \Omega$ and $(v, \eta) \in L^{2}\left(\Omega, M_{2}\right)$. This procedure requires the next two lemmas.

\section{Lemma IV.3.}

Let $h: \Omega \rightarrow \mathbf{R}^{+}$be $\mathcal{F}$-measurable, and suppose $E \sup _{0 \leq u \leq r} h(\theta(u, \cdot)$ is finite. Then $\Omega_{1}:=\left(\lim _{t \rightarrow \infty} \frac{1}{t} h(\theta(t, \cdot)=0)\right.$ is a sure event, and $\theta(t, \cdot)\left(\Omega_{1}\right) \subseteq \Omega_{1}$ for all $t \geq 0$.

Proof.

Use interpolation between delay periods and apply the discrete ergodic theorem to the $L^{1}$ function

$$
\hat{h}:=\sup _{0 \leq u \leq r} h(\theta(u, \cdot) .
$$

([M3], Lemma 5, pp. 111-113.)

\section{Lemma IV.4.}

Suppose there is a sure event $\Omega_{2}$ such that $\theta(t, \cdot)\left(\Omega_{2}\right) \subseteq \Omega_{2}$ for all $t \geq 0$, and the limit (12) exists (or equal to $-\infty$ ) for all $\omega \in \Omega_{2}$ and all $(v, \eta) \in M_{2}$. Then there is a sure event $\Omega_{3}$ such that $\theta(t, \cdot)\left(\Omega_{3}\right) \subseteq \Omega_{3}$ for all $t \geq 0$, and

$$
\lim _{t \rightarrow \infty} \frac{1}{t} \log \|X(t,(v, \eta), \omega)\|_{M_{2}}=\frac{1}{r} \lim _{k \rightarrow \infty} \frac{1}{k} \log \|X(k r,(v, \eta), \omega)\|_{M_{2}},
$$

for all $\omega \in \Omega_{3}$ and all $(v, \eta) \in M_{2}$.

Proof:

By the integrability property of Lemma IV.2, we may define $h \in L^{1}\left(\Omega, \mathbf{R}^{+} ; P\right)$ by

$$
h(\omega):=\sup _{0 \leq u \leq r} \log ^{+}\|X(u, \cdot, \omega)\|_{L\left(M_{2}\right)}, \quad \omega \in \Omega .
$$


Let $\Omega_{1}$ be the sure event in Lemma IV.3, and define $\Omega_{3}:=\Omega_{1} \cap \Omega_{2} \cap \hat{\Omega}$. The cocycle property for $X$ implies that

$$
\begin{aligned}
\frac{1}{(k+1) r} \log \|X((k+1) r,(v, \eta), \omega)\|_{M_{2}} & -\frac{1}{t} h(\theta(t, \omega)) \\
& \leq \frac{1}{t} \log \|X(t,(v, \eta), \omega)\|_{M_{2}} \\
& \leq \frac{1}{k r} h(\theta(k r, \omega))+\frac{1}{k r} \log \|X(k r,(v, \eta), \omega)\|_{M_{2}}
\end{aligned}
$$

for $k r \leq t \leq(k+1) r, \omega \in \Omega_{3},(v, \eta) \in M_{2}$. Now pass to the limit as $t \rightarrow \infty$ and $k \rightarrow \infty$ to get the required result ([M3], Lemma 6, pp. 113-114.)

Proof of Theorem IV.2. (Sketch).

Apply the Ruelle-Oseledec Theorem (Theorem IV.3) with $T(\omega):=X(r, \omega) \in L\left(M_{2}\right)$, a compact linear operator for $\omega \in \hat{\Omega} ; \tau: \Omega \rightarrow \Omega, \tau:=\theta(r, \cdot)$.

Then the cocycle property for $X$ implies that

$$
\begin{aligned}
X(k r, \omega, \cdot) & =T\left(\tau^{k-1}(\omega)\right) \circ T\left(\tau^{k-2}(\omega)\right) \circ \cdots \circ T(\tau(\omega)) \circ T(\omega) \\
& :=T^{k}(\omega)
\end{aligned}
$$

for all $\omega \in \hat{\Omega}$.

Lemma IV.2 implies that $E \log ^{+}\|T(\cdot)\|_{L\left(M_{2}\right)}<\infty$. Theorem IV.3 gives a random family of compact self-adjoint positive linear operators $\left\{\Lambda(\omega): \omega \in \Omega_{4}\right\}$ such that

$$
\Lambda(\omega):=\lim _{n \rightarrow \infty}\left[T^{n}(\omega)^{*} \circ T^{n}(\omega)\right]^{1 /(2 n)}
$$

exists in the uniform operator norm and is a positive compact operator on $M_{2}$ for $\omega \in \Omega_{4}$, a $\theta(t, \cdot)$-invariant set of full measure. Furthermore each $\Lambda(\omega)$ has a discrete spectrum

$$
e^{\mu_{1}(\omega)}>e^{\mu_{2}(\omega)}>e^{\mu_{3}(\omega)}>e^{\mu_{4}(\omega)}>\cdots
$$

where the $\mu_{i}^{\prime}$ s are distinct, with no accumulation points except possibly $-\infty$. If $\left\{\mu_{i}\right\}_{i=1}^{\infty}$ is infinite, then $\mu_{i} \downarrow-\infty$; otherwise they terminate at $\mu_{N_{0}(\omega)}=-\infty$. If $\mu_{i}(\omega)>-\infty$, then

$$
E_{1}(\omega):=M_{2}, \quad E_{i}(\omega):=\left[\oplus_{j=1}^{i-1} F_{j}(\omega)\right]^{\perp}, \quad E_{\infty}(\omega):=\operatorname{ker} \Lambda(\omega)
$$

Then

$$
E_{\infty}(\omega) \subset \cdots \subset E_{i+1}(\omega) \subset E_{i}(\omega) \cdots \subset E_{2}(\omega) \subset E_{1}(\omega)=M_{2}
$$


Note that $\operatorname{codim} E_{i}(\omega)=\sum_{j=1}^{i-1} m_{j}(\omega)<\infty$. Also

$$
\lim _{k \rightarrow \infty} \frac{1}{k} \log \|X(k r,(v, \eta), \omega)\|_{M_{2}}= \begin{cases}\mu_{i}(\omega), & \text { if }(v, \eta) \in E_{i}(\omega) \backslash E_{i+1}(\omega) \\ -\infty, & \text { if }(v, \eta) \in \operatorname{ker} \Lambda(\omega) .\end{cases}
$$

The functions

$$
\omega \mapsto \mu_{i}(\omega), \quad \omega \mapsto m_{i}(\omega), \quad \omega \mapsto N_{0}(\omega)
$$

are invariant under the ergodic shift $\theta(r, \cdot)$. Hence they take fixed values $\mu_{i}, m_{i}, N_{0}$ almost surely, respectively.

Lemma IV.4 gives a $\theta(t, \cdot)$-invariant sure event $\Omega^{*} \subseteq \Omega_{4}$ such that

$$
\begin{aligned}
\lim _{t \rightarrow \infty} \frac{1}{t} \log \|X(t,(v, \eta), \omega)\|_{M_{2}} & =\frac{1}{r} \lim _{k \rightarrow \infty} \frac{1}{k} \log \|X(k r,(v, \eta), \omega)\|_{M_{2}} \\
& =\frac{\mu_{i}}{r}=: \lambda_{i}
\end{aligned}
$$

for $(v, \eta) \in E_{i}(\omega) \backslash E_{i+1}(\omega), \omega \in \Omega^{*}, i \geq 1$.

The set $\left\{\lambda_{i}:=\frac{\mu_{i}}{r}: i \geq 1\right\}$ is the Lyapunov spectrum of (VIII).

Since the Lyapunov spectrum is discrete with no finite accumulation points, then $\left\{\lambda_{i}: \lambda_{i}>\lambda\right\}$ is finite for all $\lambda \in \mathbf{R}$.

To prove invariance of the Oseledec spaces $E_{i}(\omega)$ under the cocycle $(X, \theta)$, use the random field

$$
\lambda((v, \eta), \omega):=\lim _{t \rightarrow \infty} \frac{1}{t} \log \|X(t,(v, \eta), \omega)\|_{M_{2}} \quad(v, \eta) \in M_{2}, \quad \omega \in \Omega^{*}
$$

and the relations

$$
\begin{gathered}
E_{i}(\omega):=\left\{(v, \eta) \in M_{2}: \lambda((v, \eta), \omega) \leq \lambda_{i}\right\}, \\
\lambda(X(t,(v, \eta), \omega), \theta(t, \omega))=\lambda((v, \eta), \omega), \quad \omega \in \Omega^{*}, t \geq 0,
\end{gathered}
$$

([M3], p. 122).

The non-random nature of the Lyapunov exponents $\left\{\lambda_{i}\right\}_{i=1}^{\infty}$ of (VIII) is a consequence of the fact the $\theta$ is ergodic. (VIII) is said to be hyperbolic if $\lambda_{i} \neq 0$ for all $i \geq 1$. When (VIII) is hyperbolic, the flow satisfies a stochastic saddle-point property (or exponential dichotomy) (cf. the deterministic case with $E=C\left([-r, 0], \mathbf{R}^{d}\right), g_{i} \equiv 0, i=1, \ldots$, $m,[\mathrm{H}]$, Theorem 4.1, p. 181). 
Theorem IV.5. (Random Saddles)([M3])

Suppose the sfde (VIII) is hyperbolic. Then there exist

(a) a set $\tilde{\Omega}^{*} \in \mathcal{F}$ such that $P\left(\tilde{\Omega}^{*}\right)=1$, and $\theta(t, \cdot)\left(\tilde{\Omega}^{*}\right)=\tilde{\Omega}^{*}$ for all $t \in \mathbf{R}$, and

(b) a measurable splitting

$$
M_{2}=\mathcal{U}(\omega) \oplus \mathcal{S}(\omega), \quad \omega \in \tilde{\Omega}^{*}
$$

with the following properties:

(i) $\mathcal{U}(\omega), \mathcal{S}(\omega), \omega \in \tilde{\Omega}^{*}$, are closed linear subspaces of $M_{2}$, dim $\mathcal{U}(\omega)$ is finite and fixed independently of $\omega \in \tilde{\Omega}^{*}$.

(ii) The maps $\omega \mapsto \mathcal{U}(\omega), \omega \mapsto \mathcal{S}(\omega)$ are $\mathcal{F}$-measurable into the Grassmannian of $M_{2}$.

(iii) For each $\omega \in \tilde{\Omega}^{*}$ and $(v, \eta) \in \mathcal{U}(\omega)$ there exists $\tau_{1}=\tau_{1}(v, \eta, \omega)>0$ and a positive $\delta_{1}$, independent of $(v, \eta, \omega)$ such that

$$
\|X(t,(v, \eta), \omega)\|_{M_{2}} \geq\|(v, \eta)\|_{M_{2}} e^{\delta_{1} t}, \quad \text { for all } \quad t \geq \tau_{1}
$$

(iv) For each $\omega \in \tilde{\Omega}^{*}$ and $(v, \eta) \in \mathcal{S}(\omega)$ there exists $\tau_{2}=\tau_{2}(v, \eta, \omega)>0$ and a positive $\delta_{2}$, independent of $(v, \eta, \omega)$ such that

$$
\|X(t,(v, \eta), \omega)\|_{M_{2}} \leq\|(v, \eta)\|_{M_{2}} e^{-\delta_{2} t}, \quad \text { for all } \quad t \geq \tau_{2}
$$

(v) For each $t \geq 0$ and $\omega \in \tilde{\Omega}^{*}$,

$$
\begin{aligned}
& X(t, \omega, \cdot)(\mathcal{U}(\omega))=\mathcal{U}(\theta(t, \omega)), \\
& X(t, \omega, \cdot)(\mathcal{S}(\omega)) \subseteq \mathcal{S}(\theta(t, \omega)) .
\end{aligned}
$$

In particular, the restriction $X(t, \omega, \cdot) \mid \mathcal{U}(\omega): \mathcal{U}(\omega) \rightarrow \mathcal{U}(\theta(t, \omega))$ is a linear homeomorphism onto.

Proof.

[M3], Corollary 2, pp. 127-130. 


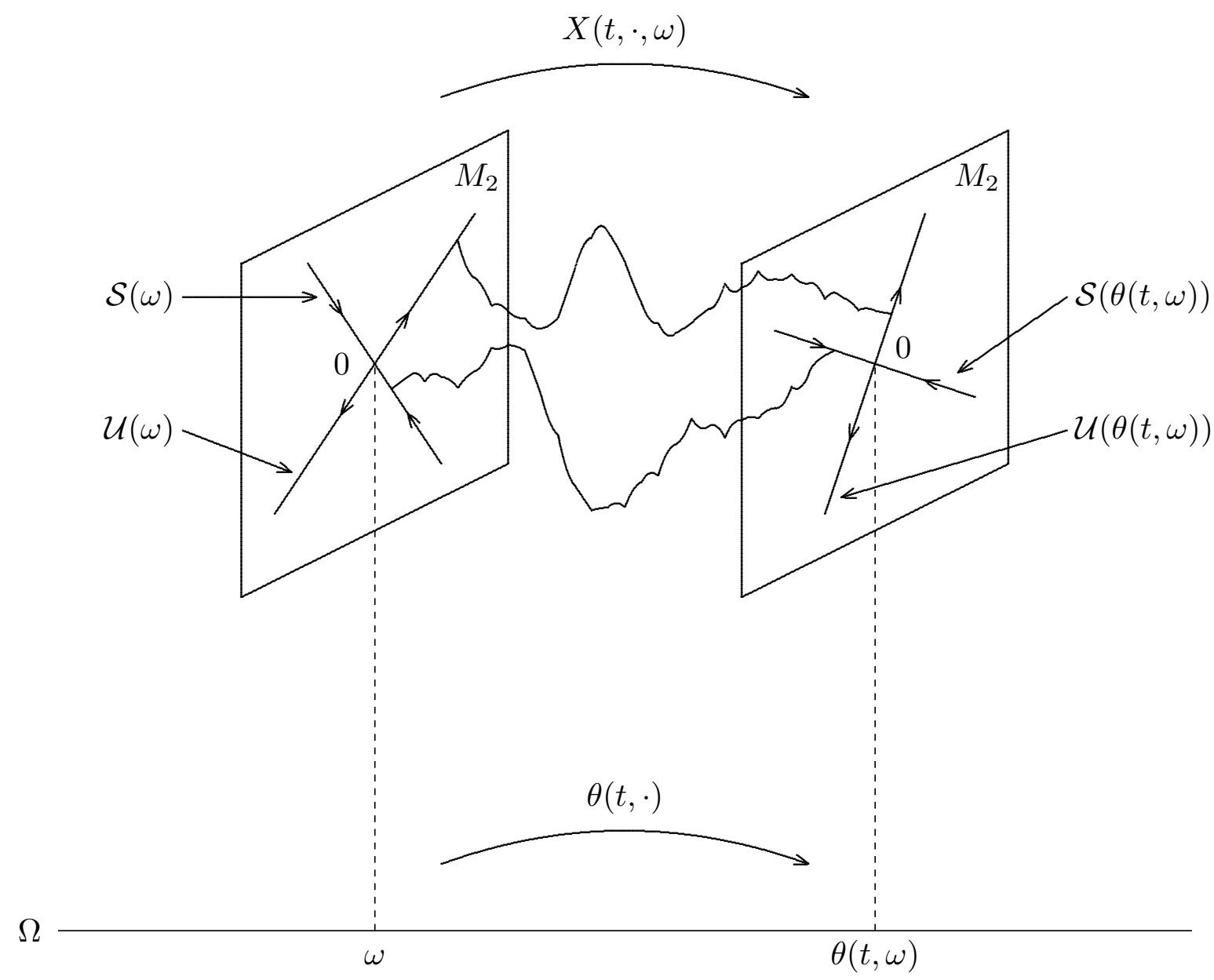

2. Regular linear systems driven by helix noise.

Consider the following linear sfde driven by semimartingale helices $N$ and $L$ :

$$
\begin{aligned}
& \left.d x(t)=\left\{\int_{[-r, 0]} \nu(t)(d s) x(t+s)\right\} d t+d N(t) \int_{-r}^{0} K(t)(s) x(t+s) d s\right\} \\
& +d L(t) x(t-), \quad t>0 \\
& \left(x(0), x_{0}\right)=(v, \eta) \in M_{2}:=\mathbf{R}^{d} \times L^{2}\left([-r, 0], \mathbf{R}^{d}\right) \text {. }
\end{aligned}
$$

In (IX), the memory is driven by a measure-valued process $\nu$ and a matrix-valued random field $K$ on a complete filtered probability space $\left(\Omega, \mathcal{F},\left(\mathcal{F}_{t}\right)_{t \in \mathbf{R}}, P\right)$.

Throughout this section we shall adopt the basic set-up and Hypotheses (R) of Section III.4. In addition, we require 


\section{Hypotheses (C)}

(i) The processes $\nu, K$ are stationary ergodic in the sense that there is a measurable ergodic $P$-preserving flow $\theta: \mathbf{R} \times \Omega \rightarrow \Omega$ such that for each $t \in \mathbf{R}, \mathcal{F}_{t}=\theta(t, \cdot)^{-1}\left(\mathcal{F}_{0}\right)$ and

$$
\begin{gathered}
\nu(t, \omega)=\nu(0, \theta(t, \omega)), \quad t \in \mathbf{R}, \omega \in \Omega, \\
K(t, \omega)=K(0, \theta(t, \omega)), \quad t \in \mathbf{R}, \omega \in \Omega .
\end{gathered}
$$

(ii) $L=M+V$, where $M$ is a continuos $\left(\mathcal{F}_{t}\right)_{t \geq 0}$-local martingale, and $V$ is a bounded variation process. The processes $N, L$ and $M$ are helices (with jointly stationary ergodic increments), viz.

$$
\begin{aligned}
N(t+h, \omega)-N(t, \omega) & =N(h, \theta(t, \omega)), \\
L(t+h, \omega)-L(t, \omega) & =L(h, \theta(t, \omega)), \\
M(t+h, \omega)-M(t, \omega) & =M(h, \theta(t, \omega)),
\end{aligned}
$$

for $t \in \mathbf{R}, \omega \in \Omega$.

Semimartingales satisfying Hypothesis (C)(ii) were studied by de Sam Lazaro and Meyer ([SM1], [SM2]), Çinlar, Jacod, Protter and Sharpe [CJPS] and Protter [P].

Equation (IX) is regular with respect to $M_{2}$ with a measurable flow $X: \mathbf{R}^{+} \times M_{2} \times$ $\Omega \rightarrow M_{2}$. This flow satisfies Theorems III.4 and the cocycle property. This is achieved via a construction in $([\mathrm{MS} 1])$ based on the following consequence of Hypothesis $(\mathrm{C})(\mathrm{ii})$ :

Theorem IV.6. ([M4], [MS1])

Suppose $M$ satisfies Hypothesis $(C)\left(\right.$ ii). Then there is an $\left(\mathcal{F}_{t}\right)_{t \geq 0}$-adapted version $\phi: \mathbf{R}^{+} \times \Omega \rightarrow \mathbf{R}^{d \times d}$ of the solution to the matrix equation

$$
\left.\begin{array}{rl}
d \phi(t) & =d M(t) \phi(t), \quad t>0 \\
\phi(0) & =I \in \mathbf{R}^{d \times d},
\end{array}\right\}
$$

and a set $\Omega_{1} \in \mathcal{F}$ such that

(i) $P\left(\Omega_{1}\right)=1$;

(ii) $\theta(t, \cdot)\left(\Omega_{1}\right) \subseteq \Omega_{1}$ for all $t \geq 0$;

(iii) $\phi\left(t_{1}+t_{2}, \omega\right)=\phi\left(t_{2}, \theta\left(t_{1}, \omega\right)\right) \phi\left(t_{1}, \omega\right)$ for all $t_{1}, t_{2} \in \mathbf{R}^{+}$and every $\omega \in \Omega_{1}$; 
(iv) $\phi(\cdot, \omega)$ is continuous on $\mathbf{R}^{+}$for every $\omega \in \Omega_{1}$.

A proof of Theorem IV.6 is given in ([M4], [MS1]), either by a double-approximation argument or via perfection techniques ([MS1], Theorem 3.1).

The existence of a discrete non-random Lyapunov spectrum $\left\{\lambda_{i}\right\}_{i=1}^{\infty}$ for the sfde (IX) is proved via Ruelle-Oseledec multiplicative ergodic theorem (Theorem IV.3), which requires the integrability property:

$$
E \sup _{0 \leq t_{1}, t_{2} \leq r} \log ^{+}\left\|X\left(t_{1}, \theta\left(t_{2}, \cdot\right), \cdot\right)\right\|_{L\left(M_{2}\right)}<\infty
$$

(Lemma IV.2). For equation (IX), the above integrability property is established under the following set of hypotheses on $\nu, K, N$ and $L$ :

\section{Hypotheses (I)}

(i)

$$
\begin{aligned}
& \sup _{-r \leq s \leq 2 r}\left|\frac{d \mu(\cdot)(s)}{d s}\right|^{2}, \quad \sup _{0 \leq t \leq 2 r} \operatorname{essup}_{t-r \leq s \leq t}\|\bar{K}(t, s, \cdot)\|^{3}, \\
& \operatorname{essup}_{0 \leq t \leq 2 r} \operatorname{essup}_{t-r \leq s \leq t}\left\|\frac{\partial}{\partial t} \bar{K}(t, s, \cdot)\right\|^{3}, \quad\{|V|(2 r, \cdot)\}^{4},
\end{aligned}
$$

are all integrable. Recall that

$$
\begin{aligned}
& \mu(\omega)(A):=\int_{0}^{\infty}|\nu(t, \omega)|\{(A-t) \cap[-r, 0]\} d t, \quad A \in \operatorname{Borel}[-r, \infty), \\
& \bar{K}(t, s, \cdot):=K(t, \omega)(s-t), \quad t \geq 0, t-r \leq s \leq t,
\end{aligned}
$$

and the measure $\mu$ has a locally (essentially) bounded density $d \mu(\cdot)(s) / d s$ with respect to Lebesgue measure on $[-r, \infty) ;|V|$ is the total variation of $V$ with respect to the Euclidean norm $\|\cdot\|$ on $\mathbf{R}^{d \times d}$.

(ii) Let $N=N^{0}+V^{0}$ where the local $\left(\mathcal{F}_{t}\right)_{t \geq 0}$-martingale $N^{0}=\left(N_{i j}^{0}\right)_{i, j=1}^{d}$ and the bounded-variation process $V^{0}=\left(V_{i j}^{0}\right)_{i, j=1}^{d}$ are such that $\left\{\left[N_{i j}^{0}\right](2 r, \cdot)\right\}^{2},\left\{\left|V_{i j}^{0}\right|(2 r, \cdot)\right\}^{4}$, $i, j=1,2, \ldots, d$, are integrable. $\left|V_{i j}^{0}\right|(2 r, \cdot)$ is the total variation of $V_{i j}^{0}$ over $[0,2 r]$.

(iii) $\left[M_{i j}\right](1) \in L^{\infty}(\Omega, \mathbf{R}), \quad i, j=1,2, \ldots, d$.

The integrability property of the cocycle $(X, \theta)$ is a consequence of

$$
E \log ^{+} \sup _{0 \leq t_{1}, t_{2} \leq r,\|(v, \eta)\| \leq 1}\left|x\left(t_{1},(v, \eta), \theta\left(t_{2}, \cdot\right)\right)\right|<\infty .
$$


The proof of the latter property uses a lengthy argument based on establishing the existence of suitable higher order moments for the coefficients of the random integral equation appearing in the proof of Theorem III.5. (See Lemmas (5.1)-(5.5) in [MS1].)

Since $\theta$ is ergodic, the multiplicative ergodic theorem (Theorem IV.3) now gives a fixed discrete set of Lyapunov exponents:

Theorem IV.7. ([M4], [MS1])

Under Hypotheses (R), (C) and (I), the statements of Theorems IV.2 and IV.5 hold true for the linear sfde (IX).

It is easy to see that the Lyapunov spectrum of (IX) does not change if one uses the state space $D\left([-r, 0], \mathbf{R}^{d}\right)$ with the supremum norm $\|\cdot\|_{\infty}([\mathrm{MS} 1]$, Remark, p. 103). 


\section{Chapter V}

\section{Stability. Examples and Case Studies}

In this chapter, we will examine several issues related to examples and case studies of linear sfde's. In particular, we will develop estimates on the "maximal exponential growth rate" for the singular noisy feedback loop introduced in Chapter I. This is done using Lyapunov functionals. We establish the existence of the stochastic semiflow and its Lyapunov spectrum for a large class of one-dimensional linear sfde's. For a variety of examples of regular linear sfde's, upper bounds on the top Lyapunov exponent $\lambda_{1}$ are obtained ([MS2]). Such bounds yield information on the almost sure asymptotic stability of the sfde. A characterization of the Lyapunov spectrum for sdde's with Poisson noise is given $([S])$.

In the literature, Lyapunov exponents for linear sode's (without memory) have been studied by many authors; e.g. [AKO], [AOP], [B], [PW1], [PW2].

Questions of asymptotic stability of general sfde's are treated in Kushner [Kus], Mizel and Trutzer [MT], Mohammed [M1], [M3], [M4], Mohammed and Scheutzow [MS1][MS3], Scheutzow [S], Kolmanovskii and Nosov [KN]. Mao ([Ma], Chapter 5) gives several results concerning the maximal exponential growth rate for sdde's driven by Kunita-type $C$-valued semimartingales. These results assume that the second-order characteristics of the driving semimartingales are time-dependent and decay to zero exponentially fast in time, uniformly in the space variable.

The results in this chapter are joint work of M. Scheutzow and the author ([MS2]).

Throughout this chapter, $W$ stands for the one-dimensional Wiener process on a complete filtered Wiener space $\left(\Omega, \mathcal{F},\left(\mathcal{F}_{t}\right)_{t \geq 0}, P\right)$.

\section{The noisy feedback loop revisited once more.}

The noisy feedback loop is modeled by the one-dimensional linear sdde:

$$
\left.\begin{array}{rl}
d x(t) & =\sigma x(t-r)) d W(t), \quad t>0 \\
\left(x(0), x_{0}\right) & =(v, \eta) \in M_{2}:=\mathbf{R} \times L^{2}([-r, 0], \mathbf{R}) .
\end{array}\right\}
$$

The delay $r$ in (I) is positive.

By Theorem III.3, (I) is singular with respect to $M_{2}$. 
Consider the more general one-dimensional linear sfde:

$$
\left.\begin{array}{rl}
d x(t) & =\int_{-r}^{0} x(t+s) d \nu(s) d W(t), \quad t>0 \\
\left.(0), x_{0}\right) & \in M_{2}:=\mathbf{R} \times L^{2}([-r, 0], \mathbf{R}),
\end{array}\right\}
$$

where $\nu$ is a fixed finite real-valued Borel measure on $[-r, 0]$.

Equation $\left(I I^{\prime}\right)$ is regular if $\nu$ has a $C^{1}$ (or even $L_{1}^{2}$ ) density with respect to Lebesgue measure on $[-r, 0]([\mathrm{MS} 1])$. If $\nu$ satisfies Theorem III.3, then $\left(I I^{\prime}\right)$ is singular. In the singular case, there is no stochastic semiflow (Theorem III.3) and we do not know whether a (discrete) set of Lyapunov exponents

$$
\lambda((v, \eta), \cdot):=\lim _{t \rightarrow \infty} \frac{1}{t} \log \left\|\left(x(t,(v, \eta)), x_{t}(\cdot,(v, \eta))\right)\right\|_{M_{2}}, \quad(v, \eta) \in M_{2},
$$

exists. The existence of Lyapunov exponents for singular equations appears to be a hard problem. However, we can still define the maximal exponential growth rate

$$
\bar{\lambda}_{1}:=\sup _{(v, \eta) \in M_{2}} \limsup _{t \rightarrow \infty} \frac{1}{t} \log \left\|\left(x(t,(v, \eta)), x_{t}(\cdot,(v, \eta))\right)\right\|_{M_{2}}
$$

for the trajectory random field $\left\{\left(x(t,(v, \eta)), x_{t}(\cdot,(v, \eta))\right): t \geq 0,(v, \eta) \in M_{2}\right\}$. In general, $\bar{\lambda}_{1}$ may depend on $\omega \in \Omega$, but $\bar{\lambda}_{1}=\lambda_{1}$ in the regular case.

In spite of the extremely erratic dependence on the initial paths of solutions of (I), it is shown in Theorem V.1 that for small noise variance, uniform almost sure global asymptotic stability still persists. For small $\sigma, \bar{\lambda}_{1} \leq-\sigma^{2} / 2+o\left(\sigma^{2}\right)$ uniformly in the initial path (Theorem V.1, and Remark (iii)). For large $|\sigma|$ and $\nu=\delta_{-r}$, one has

$$
\frac{1}{2 r} \log |\sigma|+o(\log |\sigma|) \leq \bar{\lambda}_{1} \leq \frac{1}{r} \log |\sigma|
$$

([MS2], Remark (ii) after proof of Theorem 2.3). This result is in sharp contrast with the nondelay case $(r=0)$, where $\lambda_{1}=-\sigma^{2} / 2$ for all values of $\sigma$. See Remark (ii) following the proof of Theorem V.1. The proofs of Theorems V.1 and V.2 involve very delicate constructions of new types of Lyapunov functionals on the underlying state space. 
Theorem V.1. ([MS2])

Let $\nu$ be a probability measure on $[-r, 0], r>0$, and consider the sfde

$$
\left.\begin{array}{rl}
d x(t) & =\sigma\left(\int_{[-r, 0]} x(t+s) d \nu(s)\right) d W(t), \quad t \geq 0 \\
\left.0), x_{0}\right) & =(v, \eta) \in M_{2}
\end{array}\right\}
$$

with $\sigma \in \mathbf{R},(v, \eta) \in M_{2}$. Let $x(\cdot,(v, \eta))$ be the solution of $\left(I I^{\prime}\right)$ through $(v, \eta) \in M_{2}$. Then there exists $\sigma_{0}>0$ and a continuous strictly negative nonrandom function $\phi:\left(-\sigma_{0}, \sigma_{0}\right) \rightarrow$ $\mathbf{R}^{-}$(independent of $(v, \eta) \in M_{2}$ and $\nu$ ) such that

$$
P\left(\limsup _{t \rightarrow \infty} \frac{1}{t} \log \left\|\left(x(t,(v, \eta)), x_{t}(\cdot,(v, \eta))\right)\right\|_{M_{2}} \leq \phi(\sigma)\right)=1 .
$$

for all $(v, \eta) \in M_{2}$ and all $-\sigma_{0}<\sigma<\sigma_{0}$.

\section{Remark.}

Theorem V.1 also holds for the state space $C$ with the supremum norm $\|\cdot\|_{\infty}$.

Proof of Theorem V.1. (Sketch).

For simplicity, and without loss of generality, assume that $r=1$. See Remark (i) below.

It is sufficient to consider $\left(I I^{\prime}\right)$ on $C \equiv C([-1,0], \mathbf{R})$, because $C$ is continuously embedded in $M_{2}$. Without loss of generality, assume that $\sigma>0$.

Define the Lyapunov functional $V: C \rightarrow \mathbf{R}^{+}$

$$
V(\eta):=(R(\eta) \vee|\eta(0)|)^{\alpha}+\beta R(\eta)^{\alpha}, \quad \eta \in C,
$$

where $R(\eta):=\bar{\eta}-\underline{\eta}$, the diameter of the range of $\eta, \bar{\eta}:=\sup _{-1 \leq s \leq 0} \eta(s)$ and $\underline{\eta}:=\inf _{-1 \leq s \leq 0} \eta(s)$. Fix $0<\alpha<1$. Using a lengthy computation, one can arrange for $\beta=\beta(\sigma)$ for sufficiently small $\sigma$ such that

$$
E\left(V\left({ }^{\eta} x_{1}\right)\right) \leq \delta(\sigma) V(\eta), \quad \eta \in C,
$$

where $\delta(\sigma) \in(0,1)$ is a continuous function of $\sigma$ defined near 0. Furthermore, there is a positive constant $K=K(\alpha)$ (independent of $\eta, \nu)$ such that $\delta(\sigma) \sim\left(1-K \sigma^{2}\right)$ ([MS2], pp. 12-18). The estimate (1) is hard ([MS2], pp. 12-18). Now $\left\{{ }^{\eta} x_{n}\right\}_{n=1}^{\infty}$ is a Markov 
process in $C$ (Theorem II.1). So (1) implies that $\delta(\sigma)^{-n} V\left({ }^{\eta} x_{n}\right), n \geq 1$, is a non-negative $\left(\mathcal{F}_{n}\right)$-supermartingale. Therefore there exists $Z: \Omega \rightarrow[0, \infty)$ such that

$$
\lim _{n \rightarrow \infty} \frac{V\left({ }^{\eta} x_{n}\right)}{\delta(\sigma)^{n}}=Z \quad \text { a.s. }
$$

Set $\phi(\sigma):=\frac{1}{\alpha} \log \delta(\sigma)$. Then the definition of $V$ together with (2) imply that

$$
\begin{aligned}
\varlimsup_{t \rightarrow \infty} \frac{1}{t} \log \left|{ }^{\eta} x(t)\right| & \leq \varlimsup_{n \rightarrow \infty} \frac{1}{n} \log \left[\left|{ }^{\eta} x(n)\right|+R\left({ }^{\eta} x_{n}\right)\right] \\
& =\frac{1}{\alpha} \varlimsup_{n \rightarrow \infty} \frac{1}{n} \log V\left({ }^{\eta} x_{n}\right) \leq \frac{1}{\alpha} \log \delta(\sigma)=\phi(\sigma)<0 .
\end{aligned}
$$

Note that $\delta(\sigma), \phi(\sigma)$ are independent of $\eta, \nu$, and the "domain" of $\phi$ is also independent of $\eta, \nu$.

\section{Remarks.}

(i) The choice of $\sigma_{0}$ in Theorem V.1 depends on $r$. In (I), the scaling $t \mapsto t / r$ has the effect of replacing $r$ by 1 and $\sigma$ by $\sigma \sqrt{r}$. If $\bar{\lambda}_{1}(r, \sigma)$ is the maximal exponential growth rate of (I), then $\bar{\lambda}_{1}(r, \sigma)=\frac{1}{r} \bar{\lambda}_{1}(1, \sigma \sqrt{r})$. Hence $\sigma_{0}$ decreases (like $\left.\frac{1}{\sqrt{r}}\right)$ as $r$ increases. Thus (for a fixed $\sigma$ ), a small delay $r$ tends to stabilize equation $(I)$. A large delay in (I) has a destabilizing effect. See Theorem V.2 below.

(ii) Using a Lyapunov function(al) argument, Theorem V.2 below shows that for sufficiently large $\sigma$, the singular delay equation (I) is unstable. This result is in sharp contrast with the nondelay case $r=0$, where

$$
\lim _{t \rightarrow \infty} \frac{1}{t} \log |x(t)|=-\sigma^{2} / 2<0
$$

for all $\sigma \in \mathbf{R}$. Furthermore, for fixed $r>0$, the delay equation (I) becomes more unstable as $\sigma$ increases, while the corresponding nondelay equation (for $r=0$ ) becomes more stable.

(iii) The growth rate function $\phi$ in Theorem V.1 satisfies

$$
\phi(\sigma)=-\sigma^{2} / 2+o\left(\sigma^{2}\right)
$$

as $\sigma \rightarrow 0^{+}$. This agrees with the nondelay case $r=0$. The above relation follows by suitably modifying the proof of Theorem V.1. 
Theorem V.2. ([MS2])

Consider the equation

$$
\left.\begin{array}{rl}
d x(t) & =\sigma x(t-r) d W(t), \quad t>0 \\
\left(x(0), x_{0}\right) & =(v, \eta) \in M_{2}:=\mathbf{R} \times L^{2}([-r, 0], \mathbf{R}),
\end{array}\right\}
$$

with a positive delay $r$ and $\sigma \in \mathbf{R}$. Then there exists a continuous function $\psi:(0, \infty) \rightarrow \mathbf{R}$ which is increasing to infinity such that

$$
P\left(\liminf _{t \rightarrow \infty} \frac{1}{t} \log \|\left(x(t,(v, \eta)), x_{t}(\cdot,(v, \eta)) \|_{M_{2}} \geq \psi(|\sigma|)\right)=1,\right.
$$

for all $(v, \eta) \in M_{2} \backslash\{0\}$ and all $\sigma \neq 0$. The function $\psi$ is independent of the choice of $(v, \eta) \in M_{2} \backslash\{0\}$.

\section{Remarks.}

(i) $\|\cdot\|_{M_{2}}$ can be replaced by the sup-norm $\|\cdot\|_{\infty}$ on $C$.

(ii) The proof of the theorem shows that $\psi(\sigma) \sim \frac{1}{2} \log \sigma$ for large $\sigma$.

Proof of Theorem V.2.

We will only give an outline of the proof. For more details see [MS2], pp. 20-24.

We break the proof up into two main steps.

Step 1:

We first show that

$$
P\left(\liminf _{n \rightarrow \infty} \frac{1}{n} \log \left\|\left(x(n,(v, \eta)), x_{n}(\cdot,(v, \eta))\right)\right\|_{M_{2}} \geq \psi(|\sigma|)\right)=1,
$$

for all $(v, \eta) \in M_{2} \backslash\{0\}$ and all $\sigma \neq 0$.

Without loss of generality assume that $r=1$ and $\sigma>0$.

Define the continuous Lyapunov functional $V: M_{2} \backslash\{0\} \rightarrow[0, \infty)$ by

$$
V((v, \eta))=\left(v^{2}+\sigma \int_{-1}^{0} \eta^{2}(s) d s\right)^{-1 / 4}
$$

Then an elementary (but somewhat lengthy) computation using the Gaussian nature of $\left(x(1,(v, \eta)), x_{1}(\cdot,(v, \eta))\right)$, shows that

$$
E V\left(\left(x(1,(v, \eta)), x_{1}(\cdot,(v, \eta))\right) \leq K \sigma^{-1 / 4} V((v, \eta))\right.
$$


for $(v, \eta) \neq 0$ and all $\sigma>0$, where $K$ is a positive constant suitably chosen independently of $(v, \eta) \in M_{2} \backslash\{0\}$. Set $X(t):=\left(x(t,(v, \eta)), x_{t}(\cdot,(v, \eta))\right), t \geq 0$. Then the above inequality and the Markov property imply that $K^{-n} \sigma^{n / 4} V(X(n)), n \geq 0$ is a nonnegative $\left(\mathcal{F}_{n}\right)_{n \geq 0^{-}}$ supermartingale. Hence

$$
\liminf _{n \rightarrow \infty} \frac{1}{n} \log \|X(n)\|_{M_{2}} \geq \psi(\sigma):=\frac{1}{2} \log \sigma-2 \log K
$$

almost surely. This proves a discrete version of the conclusion of the theorem.

Step 2:

We interpolate the result in Step 1 between the times $n$ and $n+1, n \geq 1$. The continuous a.s. limit

$$
\liminf _{t \rightarrow \infty} \frac{1}{t} \log \left\|\left(x(t,(v, \eta)), x_{t}(\cdot,(v, \eta))\right)\right\|_{M_{2}} \geq \psi(|\sigma|),
$$

then follows from the a.s. inequality

$$
\limsup _{n \rightarrow \infty} \frac{1}{n} Y_{n} \leq 0
$$

with

$$
Y_{n}:=-\log \inf _{n \leq t \leq n+1} \frac{\|X(t)\|_{M_{2}}}{\|X(n)\|_{M_{2}}} .
$$

For $(v, \eta) \in M_{2} \backslash\{0\}$, define

$$
Z(v, \eta):=-\inf _{0 \leq t \leq 1} \log \frac{\|X(t)\|_{M_{2}}}{\|(v, \eta)\|_{M_{2}}}
$$

By a Borel-Cantelli argument and the Markov property, the inequality $(*)$ is a consequence of the following estimate:

$$
\sup _{(v, \eta) \in M_{2} \backslash\{0\}} E\left(Z(v, \eta)^{\beta}\right)<\infty, \quad \beta>1 .
$$

The above estimate may be established using a time-change argument which exploits the Gaussian nature of the Wiener integral $\int_{0}^{t} \eta(s-1) d W(s)$ for $t \in[0,1]$. 


\section{Regular one-dimensional linear sfde's.}

The purpose of this section is to outline a general scheme for obtaining estimates on the top Lyapunov exponent for a class of one-dimensional regular linear sfde's. We then apply our scheme to specific examples within the above class. Although this will not be discussed here, the method also covers multidimensional linear sfde's with multiple delays.

A different approach in [Kus] uses Lyapunov functionals; it yields weaker estimates in all cases.

Consider the class of one-dimensional linear sfde's

$$
\left.\begin{array}{rl}
d x(t)=\left\{\nu_{1} x(t)\right. & \left.+\mu_{1} x(t-r)+\int_{-r}^{0} x(t+s) \sigma_{1}(s) d s\right\} d t \\
& +\left\{\nu_{2} x(t)+\int_{-r}^{0} x(t+s) \sigma_{2}(s) d s\right\} d M(t),
\end{array}\right\}
$$

where $r>0, \sigma_{1}, \sigma_{2} \in C^{1}([-r, 0], \mathbf{R})$, and $M$ is a continuous helix local martingale on $\left(\Omega, \mathcal{F},\left(\mathcal{F}_{t}\right)_{t \geq 0}, P\right)$ with (stationary) ergodic increments. The ergodic theorem gives the a.s. deterministic limit $\beta:=\lim _{t \rightarrow \infty} \frac{\langle M\rangle(t)}{t}$. Assume that $\beta<\infty$ and $\langle M\rangle(1) \in L^{\infty}(\Omega, \mathbf{R})$. Therefore (XVII) is regular with respect to $M_{2}$ and has a sample-continuous stochastic semiflow $X: \mathbf{R}^{+} \times M_{2} \times \Omega \rightarrow M_{2}$ (Theorem III.5). The stochastic semiflow $X$ has a fixed (non-random) Lyapunov spectrum (Theorem IV.7). Let $\lambda_{1}$ be its top exponent. We wish to develop an upper bound for $\lambda_{1}$. By the spectral theorem (Theorem IV.7, cf. Theorem IV.2), there is a shift-invariant set $\Omega^{*} \in \mathcal{F}$ of full $P$-measure and a measurable random field $\lambda: M_{2} \times \Omega \rightarrow \mathbf{R} \cup\{-\infty\}$,

$$
\lambda((v, \eta), \omega):=\lim _{t \rightarrow \infty} \frac{1}{t} \log \|X(t,(v, \eta), \omega)\|_{M_{2}}, \quad(v, \eta) \in M_{2}, \omega \in \Omega^{*}
$$

giving the Lyapunov spectrum of (XVII).

Introduce the following family of equivalent norms on $M_{2}$ :

$$
\|(v, \eta)\|_{\alpha}:=\left\{\alpha v^{2}+\int_{-r}^{0} \eta(s)^{2} d s\right\}^{1 / 2}, \quad(v, \eta) \in M_{2}, \quad \alpha>0
$$

Then

$$
\lambda((v, \eta), \omega)=\lim _{t \rightarrow \infty} \frac{1}{t} \log \|X(t,(v, \eta), \omega)\|_{\alpha}, \quad(v, \eta) \in M_{2}, \omega \in \Omega^{*}
$$

for all $\alpha>0$; i.e. the Lyapunov spectrum of (XVII) with respect to $\|\cdot\|_{\alpha}$ is independent of $\alpha>0$. 
Let $x$ be the solution of (XVII) starting at $(v, \eta) \in M_{2}$. Define

$$
\rho_{\alpha}(t)^{2}:=\|X(t)\|_{\alpha}^{2}=\alpha x(t)^{2}+\int_{t-r}^{t} x(u)^{2} d u, \quad t>0, \quad \alpha>0 .
$$

For each fixed $(v, \eta) \in M_{2}$, define the set $\Omega_{0} \in \mathcal{F}$ by $\Omega_{0}:=\left\{\omega \in \Omega: \rho_{\alpha}(t, \omega) \neq\right.$ 0 for all $t>0\}$. If $P\left(\Omega_{0}\right)=0$, then by uniqueness there is a random time $\tau_{0}$ such that a.s. $X(t,(v, \eta), \cdot)=0$ for all $t \geq \tau_{0}$. Hence $\lambda_{1}=-\infty$. Now suppose that $P\left(\Omega_{0}\right)>0$. Itô's formula implies

$$
\begin{aligned}
\log \rho_{\alpha}(t) & =\log \rho_{\alpha}(0)+\int_{0}^{t} Q_{\alpha}\left(a(u), b(u), I_{1}(u)\right) d u \\
& +\int_{0}^{t} \tilde{Q}_{\alpha}\left(a(u), I_{2}(u)\right) d\langle M\rangle(u)+\int_{0}^{t} R_{\alpha}\left(a(u), I_{2}(u)\right) d M(u),
\end{aligned}
$$

for $t>0$, a.s. on $\Omega_{0}$, where

$$
\left.\begin{array}{rl}
Q_{\alpha}\left(z_{1}, z_{2}, z_{3}\right) & :=\nu_{1} z_{1}^{2}+\sqrt{\alpha} \mu_{1} z_{1} z_{2}+\sqrt{\alpha} z_{1} z_{3}+\frac{1}{2} \frac{z_{1}^{2}}{\alpha}-\frac{1}{2} z_{2}^{2} \\
\tilde{Q}_{\alpha}\left(z_{1}, z_{3}^{\prime}\right) & :=\alpha\left(\frac{1}{2}-z_{1}^{2}\right)\left(\frac{\nu_{2}}{\sqrt{\alpha}} z_{1}+z_{3}^{\prime}\right)^{2} \\
R_{\alpha}\left(z_{1}, z_{3}^{\prime}\right) & :=\nu_{2} z_{1}^{2}+\sqrt{\alpha} z_{1} z_{3}^{\prime},
\end{array}\right\}
$$

and

$$
a(t):=\frac{\sqrt{\alpha} x(t)}{\rho_{\alpha}(t)}, \quad b(t):=\frac{x(t-r)}{\rho_{\alpha}(t)}, \quad I_{i}(t):=\frac{\int_{-r}^{0} x(t+s) \sigma_{i}(s) d s}{\rho_{\alpha}(t)}
$$

for $i=1,2, t>0$, a.s. on $\Omega_{0}$.

$$
\begin{aligned}
& \text { If }\left\|\sigma_{i}\right\|_{2}:=\left\{\int_{-r}^{0} \sigma_{i}(s)^{2} d s\right\}^{1 / 2}, i=1,2 \text {, then } \\
& \qquad\left|I_{i}(t)\right| \leq \frac{1}{\rho_{\alpha}(t)}\left(\int_{-r}^{0} x(t+s)^{2} d s\right)^{1 / 2}\left\|\sigma_{i}\right\|_{2}=\sqrt{1-a^{2}(t)}\left\|\sigma_{i}\right\|_{2},
\end{aligned}
$$

$i=1,2$, a.s. on $\Omega_{0}$. Therefore the variables $z_{1}, z_{2}, z_{3}, z_{3}^{\prime}$ in (6) must satisfy

$$
\left|z_{1}\right| \leq 1, \quad z_{2} \in \mathbf{R}, \quad\left|z_{3}\right|^{2} \leq\left(1-z_{1}^{2}\right)\left\|\sigma_{1}\right\|_{2}^{2}, \quad\left|z_{3}^{\prime}\right|^{2} \leq\left(1-z_{1}^{2}\right)\left\|\sigma_{2}\right\|_{2}^{2}
$$

Let $\tau_{1}:=\inf \left\{t>0: \rho_{\alpha}(t)=0\right\}$. Then the local martingale

$$
\int_{0}^{t \wedge \tau_{1}} R_{\alpha}\left(a(u), I_{2}(u)\right) d M(u), t>0
$$


is a time-changed (possibly stopped) Brownian motion. Since

$$
\left|R_{\alpha}\left(a(u), I_{2}(u)\right)\right| \leq\left|\nu_{2}\right|+\sqrt{\alpha}\left\|\sigma_{2}\right\|_{2}
$$

for all $u \in\left[0, \tau_{1}\right)$, a.s., then

$$
\lim _{t \rightarrow \infty} \frac{1}{t} \int_{0}^{t \wedge \tau_{1}} R_{\alpha}\left(a(u), I_{2}(u)\right) d M(u)=0 \quad \text { a.s. }
$$

Divide (5) by $t$ and let $t \rightarrow \infty$, to get

$\lambda((v, \eta), \omega) \leq \limsup _{t \rightarrow \infty} \frac{1}{t} \int_{0}^{t} Q_{\alpha}\left(a(u), b(u), I_{1}(u)\right) d u+\limsup _{t \rightarrow \infty} \frac{1}{t} \int_{0}^{t} \tilde{Q}_{\alpha}\left(a(u), I_{2}(u)\right) d\langle M\rangle(u)$.

a.s. on $\Omega_{0}$, for all $\alpha>0$.

We wish to develop upper bounds for $\lambda_{1}$ in the following special cases.

Our first example is the one-dimensional linear sfde:

$$
d x(t)=\left\{\nu_{1} x(t)+\mu_{1} x(t-r)\right\} d t+\left\{\int_{-r}^{0} x(t+s) \sigma_{2}(s) d s\right\} d W(t), \quad t>0
$$

with real constants $\nu_{1}, \mu_{1}$, and $\sigma_{2} \in C^{1}([-r, 0], \mathbf{R})$. (VII) is a special case of (XVII). Hence it is regular with respect to $M_{2}$. Observe that (VII) has a smooth memory in the white-noise term. Indeed the process $\int_{-r}^{0} x(t+s) \sigma_{2}(s) d s$ has $C^{1}$ paths in $t$. Hence the stochastic differential $d W$ in (VII) may be interpreted in the Itô or Stratonovich sense without changing the solution $x$.

Theorem V.3. ([MS2])

Suppose $\lambda_{1}$ is the top a.s. Lyapunov exponent of (VII). Define the function

$$
\theta(\delta, \alpha):=-\delta+\left(\nu_{1}+\delta+\frac{1}{2} \alpha \mu_{1}^{2} e^{2 \delta r}+\frac{1}{2 \alpha}\right) \vee\left(\frac{\alpha}{2}\left\|\sigma_{2}\right\|_{2}^{2} e^{2 \delta^{+} r}\right)
$$

for all $\alpha \in \mathbf{R}^{+}, \delta \in \mathbf{R}$, where $\delta^{+}:=\max \{\delta, 0\}$.

Then

$$
\lambda_{1} \leq \inf \left\{\theta(\delta, \alpha): \delta \in \mathbf{R}, \alpha \in \mathbf{R}^{+}\right\} .
$$


Proof.

In the right-hand-side of (9), set $M=W$ and maximize the function $Q_{\alpha}\left(z_{1}, z_{2}, z_{3}\right)+$ $\tilde{Q}_{\alpha}\left(z_{1}, z_{3}^{\prime}\right)$ over its domain of definition. This gives the upper bound

$$
\lambda_{1} \leq\left(\nu_{1}+\frac{1}{2} \mu_{1}^{2}+\frac{1}{2 \alpha}\right) \vee\left(\frac{\alpha}{2}\left\|\sigma_{2}\right\|_{2}^{2}\right)
$$

Next we shift the Lyapunov spectrum of (VII) by an arbitrary amount $\delta \in \mathbf{R}$. We do this by setting $y(t):=\mathrm{e}^{\delta t} x(t), t \geq-r$, for a fixed $\delta \in \mathbf{R}$. Then $y$ solves a linear sfde of the type (VII) where $\nu_{1}, \mu_{1}, \sigma_{2}$ are replaced (respectively) by $\nu_{1}+\delta, \mu_{1} \mathrm{e}^{\delta r}$ and $\hat{\sigma}_{2}$, where $\hat{\sigma}_{2}(s):=\mathrm{e}^{-\delta s} \cdot \sigma_{2}(s), \quad s \in[-r, 0]$.

Now apply the above estimate to the top exponent of the transformed equation, and then minimize over $\alpha, \delta$ to get (10) ([MS2], Theorem 4.1).

The next corollary shows that the estimate in Theorem V.3 reduces to a well-known estimate in the deterministic case $\sigma_{2} \equiv 0$ ([Ha], pp.17-18).

Corollary V.3.1. ([MS2])

In (VII), suppose $\mu_{1} \neq 0$ and let $\delta_{0}$ be the unique real solution of the transcendental equation

$$
\nu_{1}+\delta+\left|\mu_{1}\right| e^{\delta r}=0
$$

Then

$$
\lambda_{1} \leq-\delta_{0}+\frac{1}{2} \frac{\left\|\sigma_{2}\right\|_{2}^{2}}{\left|\mu_{1}\right|} e^{\left|\delta_{0}\right| r}
$$

If $\mu_{1}=0$ and $\nu_{1} \geq 0$, then $\lambda_{1} \leq \frac{1}{2}\left(\nu_{1}+\sqrt{\nu_{1}^{2}+\left\|\sigma_{2}\right\|_{2}^{2}}\right)$. If $\mu_{1}=0$ and $\nu_{1}<0$, then $\lambda_{1} \leq \nu_{1}+\frac{1}{2}\left\|\sigma_{2}\right\|_{2} e^{-\nu_{1} r}$.

Proof.

Suppose $\mu_{1} \neq 0$. Denote by $f(\delta), \delta \in \mathbf{R}$, the left-hand-side of (11). Then $f(\delta)$ is an increasing function of $\delta$. $f$ has a unique real zero $\delta_{0}$. Using (10), we may put $\delta=\delta_{0}$ and $\alpha=\left|\mu_{1}\right|^{-1} e^{-\delta_{0} r}$ in the expression for $\theta(\delta, \alpha)$. This gives (12).

Suppose $\mu_{1}=0$. Put $\delta=\left(-\nu_{1}\right)^{+}$in $\theta(\delta, \alpha)$ and minimize the resulting expression over all $\alpha>0$. This proves the last two assertions of the corollary ([MS2], pp. 35-36). 


\section{Remarks.}

(i) The upper bounds for $\lambda_{1}$ in Theorem (V.3) and Corollary V.3.1 agree with the corresponding bounds in the deterministic case (for $\mu_{1} \geq 0$ ), but are not optimal when $\mu_{1}=0$ and $\sigma_{2}$ is strictly positive and sufficiently small; cf. Theorem V.1 for small $\left\|\sigma_{2}\right\|_{2}$.

(ii) It is an interesting problem to develop the asymptotics of $\lambda_{1}$ for small delays $r \downarrow 0$ ?

Our second example is the stochastic delay equation

$$
d x(t)=\left\{\nu_{1} x(t)+\mu_{1} x(t-r)\right\} d t+x(t) d M(t), \quad t>0,
$$

where $M$ is the continuous helix local martingale appearing in (XVII) and satisfying the conditions therein. Hence (XVIII) is regular with respect to $M_{2}$. Theorem V.4 below gives an estimate on its top exponent.

Theorem V.4. ([MS2])

In (XVIII), define $\delta_{0}$ as in Corollary V.3.1. Then the top a.s. Lyapunov exponent $\lambda_{1}$ of (XVIII) satisfies

$$
\lambda_{1} \leq-\delta_{0}+\frac{\beta}{16}
$$

Proof.

First maximize the following functions separately over their appropriate ranges:

$$
\begin{aligned}
Q_{\alpha}\left(z_{1}, z_{2}\right) & :=\nu_{1} z_{1}^{2}+\sqrt{\alpha} \mu_{1} z_{1} z_{2}+\frac{1}{2} \frac{z_{1}^{2}}{\alpha}-\frac{1}{2} z_{2}^{2}, \\
\tilde{Q}_{\alpha}\left(z_{1}\right) & :=\left(\frac{1}{2}-z_{1}^{2}\right) z_{1}^{2}, \quad\left|z_{1}\right| \leq 1, z_{2} \in \mathbf{R} .
\end{aligned}
$$

Then use an exponential shift of the Lyapunov spectrum by an amount $\delta$ (cf. proof of Theorem V.3). Minimize the resulting bound over all $\alpha$ (for fixed $\delta$ ) and then over all $\delta \in \mathbf{R}$. This minimum is attained if $\delta$ solves the transcendental equation (11). Hence the conclusion of the theorem holds ([MS2]).

\section{Remarks.}

(i) The above estimate for $\lambda_{1}$ is sharp in the deterministic case $\beta=0$ and $\mu_{1} \geq 0$, but is not sharp when $\beta \neq 0$; e.g. $M=W$ and $\mu_{1}=0$.

(ii) When $M=\nu_{2} W$ for a fixed real $\nu_{2}$, the above bound may be considerably sharpened as in Theorem V.5 below. The sdde in this theorem corresponds to the model of dye circulation in the blood stream given by equation (VI) of Chapter I, Section 1 (cf. Bailey and Williams [BW]; Lenhart and Travis [LT]). 
Theorem V.5. ([MS2])

For the equation

$$
d x(t)=\left\{\nu_{1} x(t)+\mu_{1} x(t-r)\right\} d t+\nu_{2} x(t) d W(t), \quad t>0,
$$

set

$$
\phi(\delta):=-\delta+\frac{1}{4 \nu_{2}^{2}}\left[\left(\left|\mu_{1}\right| e^{\delta r}+\nu_{1}+\delta+\frac{1}{2} \nu_{2}^{2}\right)^{+}\right]^{2},
$$

for $\nu_{2} \neq 0$. Then

$$
\lambda_{1} \leq \inf _{\delta \in \mathbf{R}} \phi(\delta) .
$$

In particular, if $\delta_{0}$ is the unique solution of the equation

$$
\nu_{1}+\delta+\left|\mu_{1}\right| e^{\delta r}+\frac{1}{2} \nu_{2}^{2}=0,
$$

then $\lambda_{1} \leq-\delta_{0}$.

Proof.

Maximize

$$
Q_{\alpha}\left(z_{1}, z_{2}, 0\right)+\tilde{Q}_{\alpha}\left(z_{1}, 0\right)=\left(\nu_{1}+\frac{1}{2 \alpha}+\frac{\nu_{2}^{2}}{2}\right) z_{1}^{2}+\sqrt{\alpha} \mu_{1} z_{1} z_{2}-\frac{1}{2} z_{2}^{2}-\nu_{2}^{2} z_{1}^{4}
$$

over $\left|z_{1}\right| \leq 1, z_{2} \in \mathbf{R}$, and then minimize the resulting bound for $\lambda_{1}$ over $\alpha>0$. This gives

$$
\lambda_{1} \leq \frac{1}{4 \nu_{2}^{2}}\left[\left(\nu_{1}+\left|\mu_{1}\right|+\frac{\nu_{2}^{2}}{2}\right)^{+}\right]^{2} .
$$

The first assertion of the theorem follows from the above estimate by applying an exponential shift to (VI). The last assertion of the theorem is obvious.

Problem:

$$
\text { Is } \lambda_{1}=\inf _{\delta \in \mathbf{R}} \phi(\delta) ?
$$

\section{Remark.}

The estimate in Theorem V.5 agrees with the nondelay case $\mu_{1}=0$ whereby $\lambda_{1}=$ $\nu_{1}-\frac{1}{2} \nu_{2}^{2}=\inf _{\delta \in \mathbf{R}} \phi(\delta)$. Cf. also [AOP], [B] and [AKO]. 


\section{An sdde with Poisson noise.}

Consider the one-dimensional linear delay equation

$$
\left.\begin{array}{rl}
d x(t) & =x((t-1)-) d N(t), \quad t>0 \\
x_{0} & =\eta \in D:=D([-1,0], \mathbf{R}) .
\end{array}\right\}
$$

The process $N(t) \in \mathbf{R}$ is a Poisson process with i.i.d. inter-arrival times $\left\{T_{i}\right\}_{i=1}^{\infty}$ which are exponentially distributed with the same parameter $\mu$. The jumps $\left\{Y_{i}\right\}_{i=1}^{\infty}$ of $N$ are i.i.d. and independent of all the $T_{i}$ 's. Let $j(t):=\sup \left\{j \geq 0: \sum_{i=1}^{j} T_{i} \leq t\right\}$. Then $N(t)=\sum_{i=1}^{j(t)} Y_{i}$. Equation (V) can be solved pathwise in forward steps of lengths 1, using the almost sure relation

$$
x^{\eta}(t)=\eta(0)+\sum_{i=1}^{j(t)} Y_{i} x\left(\left(\sum_{j=1}^{i} T_{j}-1\right)-\right) \quad \text { a.s. }
$$

The trajectory $\left\{x_{t}: t \geq 0\right\}$ of $(\mathrm{V})$ is a Markov process in the state space $D$ (with the supremum norm $\left.\|\cdot\|_{\infty}\right)$. Furthermore, the above relation implies that $(\mathrm{V})$ is regular with respect to $D$; i.e., it admits a measurable flow $X: \mathbf{R}^{+} \times D \times \Omega \rightarrow D$ with $X(t, \cdot, \omega):=$ ${ }^{\eta} x_{t}(\cdot, \omega)$, continuous linear in $\eta$ for all $t \geq 0$ and almost all $\omega \in \Omega$ (cf. the singular equation (I)).

The almost sure Lyapunov spectrum of (V) may be characterized directly (without appealing to the Oseledec Theorem) by interpolating between the following sequence of random times:

$$
\begin{aligned}
\tau_{0}(\omega) & :=0 \\
\tau_{1}(\omega) & :=\inf \left\{n \geq 1: \sum_{j=1}^{k} T_{j} \notin[n-1, n] \quad \text { for all } k \geq 1\right\} \\
\tau_{i+1}(\omega) & :=\inf \left\{n>\tau_{i}(\omega): \sum_{j=1}^{k} T_{j} \notin[n-1, n] \text { for all } k \geq 1\right\}, \quad i \geq 1 .
\end{aligned}
$$

It is easy to see that $\left\{\tau_{1}, \tau_{2}-\tau_{1}, \tau_{3}-\tau_{2}, \cdots\right\}$ are i.i.d. and $E \tau_{1}=\mathrm{e}^{\mu}$.

Theorem V.6. ([S], [MS2])

Let $\xi \in D$ be the constant path $\xi(s)=1$ for all $s \in[-1,0]$. Suppose

$E \log \left\|X\left(\tau_{1}(\cdot), \xi, \cdot\right)\right\|_{\infty}$ exists (possibly $=+\infty$ or $-\infty$ ). Then the almost sure Lyapunov spectrum

$$
\lambda(\eta):=\lim _{t \rightarrow \infty} \frac{1}{t} \log \|X(t, \eta, \omega)\|_{\infty}, \quad \eta \in D, \omega \in \Omega
$$


of $(V)$ is $\left\{-\infty, \lambda_{1}\right\}$ where $\lambda_{1}=e^{-\mu} E \log \left\|X\left(\tau_{1}(\cdot), \xi, \cdot\right)\right\|_{\infty}$. In fact,

$$
\lim _{t \rightarrow \infty} \frac{1}{t} \log \|X(t, \eta, \omega)\|_{\infty}= \begin{cases}\lambda_{1}, & \eta \notin \operatorname{Ker} X\left(\tau_{1}(\omega), \cdot, \omega\right) \\ -\infty, & \eta \in \operatorname{Ker} X\left(\tau_{1}(\omega), \cdot \omega\right) .\end{cases}
$$

Proof.

The i.i.d. sequence

$$
S_{i}:=\frac{\left\|\left(X\left(\tau_{i}, \xi, \cdot\right)\right)\right\|}{\left\|\left(X\left(\tau_{i-1}, \xi, \cdot\right)\right)\right\|} \quad i=1,2, \ldots
$$

and the law of large numbers give

$$
\lim _{n \rightarrow \infty} \frac{1}{\tau_{n}} \log \left\|\left(X\left(\tau_{n}, \xi, \omega\right)\right)\right\|=e^{-\mu}\left(E \log S_{1}\right)
$$

for almost all $\omega \in \Omega$.

Now interpolate between the times $\tau_{1}, \tau_{2}, \tau_{3}, \cdots$ to get the continuous limit $\lim _{t \rightarrow \infty} \frac{1}{t} \log \|X(t, \eta, \omega)\|_{\infty}$ ([MS2], Proof of Theorem 3.1). 


\section{Chapter VI}

\section{Miscellanea}

\section{Malliavin calculus of sfde's.}

Our main objective in this section is to establish the existence of smooth densities for solutions of $\mathbf{R}^{d}$-valued sfde's of the form

$$
d x(t)=H\left(t, x_{t}\right) d t+g(t, x(t-r)) d W(t) .
$$

In the above equation, $W$ is an $m$-dimensional Wiener process, $r$ is a positive time delay, $H$ is a map $[0, \infty) \times C\left([-r, 0], \mathbf{R}^{d}\right) \rightarrow \mathbf{R}^{d}$ and $g:[0, \infty) \times \mathbf{R}^{d} \rightarrow \mathbf{R}^{d \times m}$ is a $d \times m$ matrix-valued function. For each $t \geq 0$, the $d \times d$-matrix $g(t, x) g(t, x)^{*}$ has degeneracies of polynomial order as $x$ runs on a hypersurface in $\mathbf{R}^{d}$. Our analysis of (XIX) gives a very general criterion for the hypoellipticity of a class of degenerate parabolic secondorder time-dependent differential operators with space-independent principal parts. More generally, the analysis of the sfde (XIX) works when $H$ is replaced by a non-anticipating functional which may depend on the whole history of the path ([BM4]).

The special case $H \equiv 0$ was studied in [BM1]. In [BM1], it is shown that the solution $x(t)$ has a smooth density with respect to Lebesgue measure on $\mathbf{R}^{d}$, under the hypothesis that the matrix $g(t, x) g(t, x)^{*}$ degenerates like $|x|^{2}$ near 0 (e.g. when $g(t, \cdot)$ is linear.) Our approach to (XIX) (as in [BM1]) uses the Malliavin calculus. However there are difficulties, which we outline below.

\section{Difficulties.}

(i) The infinitesimal generator of the trajectory Feller process $\left\{x_{t}: t \geq 0\right\}$ is a highly degenerate second-order differential operator on the state space: Its principal part degenerates on a surface of finite codimension (Theorem II.3). Hence one cannot use existing techniques from pde's.

(ii) The analysis by the Malliavin calculus requires the derivation of probabilistic lower bounds on the Malliavin covariance matrix of the solution $x$. These bounds are difficult because there is no stochastic flow in the singular case (Theorem III.3). Cf. the sode case, where the stochastic flow is invertible. See work by Kusuoka and Stroock in the uniformly elliptic case $([\mathrm{KS} 1])$.

(iii) The form of the Malliavin covariance allows only a polynomial (finite-type) rate of degeneracy near a hypersurface, coupled with limited contact of the initial path 
with the hypersurface. Note the sode case where degeneracies of infinite type are compatible with hypellipticity ([BM2]).

\section{Hypotheses (S).}

(i) $W:[0, \infty) \times \Omega \rightarrow \mathbf{R}^{m}$ is standard $m$-dimensional Wiener process, defined on a complete filtered probability space $\left(\Omega, \mathcal{F},\left(\mathcal{F}_{t}\right)_{t \geq 0}, P\right)$.

(ii) $g:[0, \infty) \times \mathbf{R}^{d} \rightarrow \mathbf{R}^{d \times m}$ is a continuous map into the space of $d \times m$ matrices, with bounded Fréchet derivatives of all orders in the space variable.

(iii) $r$ is a positive real number, and $\eta:[-r, 0] \rightarrow \mathbf{R}^{d}$ is a continuous initial path.

(iv) $H:[0, \infty) \times C \rightarrow \mathbf{R}^{d}$ is a globally bounded continuous map with all partial Fréchet derivatives of $H(t, \eta)$ with respect to $\eta$, globally bounded in $(t, \eta) \in \mathbf{R}^{+} \times C$. Think of $H\left(t, \xi_{t}\right)$ as a smooth $\mathbf{R}^{d}$-valued functional in $\xi \in C\left([-r, t], \mathbf{R}^{d}\right)$. Denote its Fréchet derivative with respect to $\xi \in C\left([-r, t], \mathbf{R}^{d}\right)$ by $H_{\xi}(t, \xi)$. Set

$$
\alpha_{t}:=\sup \left\{\left\|H_{\xi}(u, \xi)\right\|: u \in[0, t], \xi \in C\left([-r, u], \mathbf{R}^{d}\right)\right\}, \quad t>0
$$

and

$$
\alpha_{\infty}:=\sup \left\{\left\|H_{\xi}(u, \xi)\right\|: u \in[0, \infty), \xi \in C\left([-r, u], \mathbf{R}^{d}\right)\right\}
$$

where $\left\|H_{\xi}(u, \xi)\right\|$ is the operator norm of the partial Fréchet derivative $H_{\xi}(u, \xi)$ : $C\left([-r, u], \mathbf{R}^{d}\right) \rightarrow \mathbf{R}^{d}$.

Theorem VI.1. ([BM4])

Assume Hypotheses (S) for the sfde (XIX). Suppose there exist positive constants $\rho$, $\delta$, an integer $p \geq 2$ and a function $\phi:[0, \infty) \times \mathbf{R}^{d} \rightarrow \mathbf{R}$ satisfying the following conditions:

(i)

$$
g(t, x) g(t, x)^{*} \geq\left\{\begin{array}{cl}
|\phi(t, x)|^{p} I, & |\phi(t, x)|<\rho \\
\delta I, & |\phi(t, x)| \geq \rho
\end{array}\right.
$$

for $(t, x) \in[0, \infty) \times \mathbf{R}^{d}$.

(ii) $\phi(t, x)$ is $C^{1}$ in $t$ and $C^{2}$ in $x$, with bounded first derivatives in $(t, x)$ and bounded second derivatives in $x \in \mathbf{R}^{d}$.

(iii) There is a positive constant c such that

$$
\|\nabla \phi(t, x)\| \geq c>0
$$


for all $(t, x) \in[0, \infty) \times \mathbf{R}^{d}$, with $|\phi(t, x)| \leq \rho$. In (2), $\nabla$ denotes the gradient operator with respect to the space variable $x \in \mathbf{R}^{d}$.

(iv) The set $\{s: s \in[-r, 0], \phi(s+r, \eta(s))=0\}$ has Lebesgue measure less than $\left(3 \alpha_{\infty}\right)^{-1} \wedge r$.

Define $s_{0} \in[-r, 0]$ by

$$
s_{0}:=\sup \left\{s \in[-r, 0]: \int_{-r}^{s} \phi(u+r, \eta(u))^{2} d u=0\right\} .
$$

Then for all $t>s_{0}+r$ the solution $x(t)$ of $(X I X)$ is absolutely continuous with respect to d-dimensional Lebesgue measure, and has a $C^{\infty}$ density.

\section{Remark.}

Condition (iv) of Theorem VI.1 is equivalent to the following:

(iv) ${ }^{\prime}$ There is a positive number $\delta_{0}$ such that $\delta_{0}<\left(3 \alpha_{\infty}\right)^{-1} \wedge r$ and for every Borel set $J_{0} \subseteq[-r, 0]$ of Lebesgue measure $\delta_{0}$ the following holds

$$
\int_{J_{0}} \phi(t+r, \eta(t))^{2} d t>0 .
$$

Theorem VI.2. ([BM4])

In the sfde

$$
\left.\begin{array}{c}
d y(t)=H\left(t, y_{t}\right) d t+F(t) d W(t), \quad t>a \\
y(t)=x(t), \quad a-r \leq t \leq a, \quad a \geq r
\end{array}\right\}
$$

suppose that $F:[a, \infty) \rightarrow \mathbf{R}^{d \times m}$ and $x:[a-r, a] \rightarrow \mathbf{R}^{d}$ are continuous. Assume that $H$ satisfies regularity hypotheses analogous to $(S)$. For each $t>a$, let

$$
\alpha_{t}^{\prime}:=\sup \left\{\left\|H_{\xi}(u, \xi)\right\|: u \in[0, t], \xi \in C\right\} .
$$

Suppose there exists a positive number $\delta^{*}<1 /\left(3 \alpha_{t}^{\prime}\right)$ such that

$$
\int_{t-\delta^{*}}^{t} \mu_{1}(s) d s>0
$$

where $\mu_{1}(s), s \geq a$, is the smallest eigenvalue of the non-negative definite matrix $F(s) F(s)^{*}$. Then for each $t>$ a the solution $y(t)$ of $(X X)$ has an absolutely continuous distribution with respect to d-dimensional Lebesgue measure and has a $C^{\infty}$ density.

In (XX), suppose $H(t, y)=h(t, y(t))$ for some Lipschitz function $h: \mathbf{R}^{+} \times \mathbf{R}^{d} \rightarrow \mathbf{R}^{d}$. Then $y$ is a (time-inhomogeneous) diffusion process. In this case the proof of Theorem VI.2 gives the following pde result: 
Theorem VI.3. ([BM4])

For each $t>0$, let $A(t)=\left[a_{i j}(t)\right]_{i, j=1}^{d}$ denote a symmetric non-negative definite $d \times d$-matrix. Let $\mu_{2}(t)$ be the smallest eigenvalue of $A(t)$. Assume the following:

(i) The map $t \longmapsto A(t)$ is continuous.

(ii) There exists $T>0$ such that $\int_{0}^{T} \mu_{2}(s) d s>0$.

(iii) The functions $b_{i}, i=1, \ldots, d, c: \mathbf{R}^{+} \times \mathbf{R}^{d} \rightarrow \mathbf{R}$ are bounded, jointly continuous in $(t, x)$ and have partial derivatives of all orders in $x$, all of which are bounded in $(t, x)$. Let $T_{0}:=\sup \left\{T>0: \int_{0}^{T} \mu_{2}(s) d s=0\right\}$, and let $L_{t, x}$ denote the differential operator

$$
L_{t, x}:=\frac{1}{2} \sum_{j=1}^{d} a_{i j}(t) \frac{\partial^{2}}{\partial x_{i} \partial x_{j}}+\sum_{i=1}^{d} b_{i}(t, x) \frac{\partial}{\partial x_{i}}+c(t, x) .
$$

Then the parabolic equation $\frac{\partial u}{\partial t}=L_{t, x} u$ has a fundamental solution $\Gamma(t, x, y)$ defined on $\left(T_{0}, \infty\right) \times \mathbf{R}^{2 d}$, which is $C^{1}$ in $t$ and $C^{\infty}$ in $(x, y)$. Furthermore, if the coefficients $a_{i j}(t), b_{i}(t, x), c(t, x), i, j=1, \ldots, d$, are $C^{\infty}$ in $(t, x)$, and

$$
\lim _{t \rightarrow T_{0}+}\left(t-T_{0}\right) \log \left\{\int_{T_{0}}^{t} \mu_{2}(s) d s\right\}=0
$$

then $\frac{\partial}{\partial t}-L_{t, x}$ is a hypoelliptic operator on $\left(T_{0}, \infty\right) \times \mathbf{R}^{d}$; (viz. if $\phi$ is a distribution on $\left(T_{0}, \infty\right) \times \mathbf{R}^{d}$ such that $\left(\frac{\partial}{\partial t}-L_{t, x}\right) \phi$ is $C^{\infty}$, then $\phi$ is also $C^{\infty}$.)

The mean ellipticity hypothesis in (ii) is much weaker than classical pointwise ellipticity that is usually assumed in the pde literature.

\section{Problem.}

Can Theorem VI.3 be proved using existing pde's techniques?

Proof of Theorem VI.1. (Outline).

Our objective is to get good probabilistic lower bounds on the Malliavin covariance matrix of the solution $x(t)$ of (XIX); (cf. the "conditioning argument" in [BM4] which uses $(\mathrm{XX})$ ). We establish the above-mentioned lower bounds using the following steps: 
Step 1.

In (XIX), we use piecewise linear approximations of $W$ to compute the Malliavin covariance matrix $C(T)$ of $x(T)$ as

$$
C(T)=\int_{0}^{T} Z(u) g(u, x(u-r)) g(u, x(u-r))^{*} Z(u)^{*} d u
$$

where the $(d \times d)$-matrix-valued process $Z:[0, T] \times \Omega \rightarrow \mathbf{R}^{d \times d}$ satisfies the advanced anticipating Stratonovich integral equation

$$
Z(t)=I+\int_{T \wedge(t+r)}^{T} Z(u) D_{2} g(u, x(u-r))(\cdot) \circ d W(u)+\int_{t}^{T} Z(u)\left[\left\{H_{x}(u, x)^{*}(\cdot)\right\}^{\prime}(t)\right]^{*} d u
$$

for $0 \leq t \leq T$. In the above integral equation, $H_{x}(u, x)$ is the Fréchet partial derivative of the map $(u, x) \mapsto H\left(u, x_{u}\right)$ with respect to $x \in C\left([-r, u], \mathbf{R}^{d}\right) ;$ and $D_{2} g(t, v): \mathbf{R}^{d} \rightarrow \mathbf{R}^{d \times m}$ is the partial (Fréchet) derivative of $g$ with respect to $v \in \mathbf{R}^{d}$. Let $W^{1,2}$ be the CameronMartin subspace of $C\left([-r, u], \mathbf{R}^{d}\right)$, and denote by $H_{x}(u, x)^{*}$ the Hilbert-space adjoint of the restriction $H_{x}(u, x) \mid W^{1,2}: W^{1,2} \rightarrow \mathbf{R}^{d}$.

We solve the above integral equation as follows.

Start with the terminal condition $Z(T)=I$. On the last delay period $[(T-r) \vee 0, T]$ define $Z$ to be the unique solution of the linear integral equation

$$
Z(t)=I+\int_{t}^{T} Z(u)\left[\left\{H_{x}(u, x)^{*}(\cdot)\right\}^{\prime}(t)\right]^{*} d u
$$

for a.e. $t \in((T-r) \vee 0, T)$. When $T>r$, use successive approximations to solve the anticipating integral equation, treating the stochastic integral as a predefined random forcing term. This gives a unique solution of the integral equation by successive backward steps of length $r$. The matrix $Z(t)$ need not be invertible for small $t$. It is interesting to compare $Z(t)$ with the analogous process for the diffusion case (sode). In the latter case $Z(t)$ is invertible for all $t$ and anticipating integrals are not needed.

Step 2.

Since $H_{x}(u, x)$ is globally bounded in $(u, x)$, then so is $\left[H_{x}(u, x)^{*}(\cdot)\right]^{\prime}(t)$ in $(u, x, t)$ ([BM4], Lemma 3.3). Hence we can choose a deterministic time $t_{0}<T$ sufficiently close to $T$ such that almost surely $Z(t)$ is invertible and $\left\|Z(t)^{-1}\right\| \leq 2$ for a.e. $t \in\left(t_{0}, T\right]([\mathrm{BM} 4]$, Lemma 3.4). 
Step 3.

The above lower bound on $\|Z(t)\|$ and the representation of $C(T)$ imply that

$$
\operatorname{det} C(T) \geq\left[\frac{1}{4} \int_{t_{0}}^{T}\{\hat{g}(u, x(u-r))\}^{2} d u\right]^{d} \quad \text { a.s. }
$$

where $\hat{g}(u, v):=\inf \left\{\left|g(u, v)^{*}(e)\right|: e \in \mathbf{R}^{d}, \quad|e|=1\right\}$, for all $u \geq 0, v \in \mathbf{R}^{d}$.

Step 4 .

Note the

Propagation Lemma.

Let $-r<a<b<a+r$. Then the statement

$$
P\left(\int_{a}^{b}|\phi(u+r, x(u))|^{2} d u<\epsilon\right)=o\left(\epsilon^{k}\right)
$$

as $\epsilon \rightarrow 0+$ for every $k \geq 1$,

implies that

$$
P\left(\int_{a+r}^{b+r}|\phi(u+r, x(u))|^{2} d u<\epsilon\right)=o\left(\epsilon^{k}\right)
$$

as $\epsilon \rightarrow 0+$ for every $k \geq 1$.

The proof of the above lemma uses Itô's formula, the lower bound on $\|\nabla \phi\|$, the polynomial degeneracy condition, and the Kusuoka-Stroock $\epsilon^{1 /(18)}$-lemma! ([BM4], Lemma $4.2)$.

Step 5 .

Using (iv)' of the remark following Theorem VI.1, we may successively apply Step 4 to propagate the "limited contact" hypothesis on the initial path $\eta$ ((i) of Theorem VI.1). This yields the estimate:

$$
P\left(\int_{t_{0}}^{T}|\phi(u, x(u-r))|^{2} d u<\epsilon\right)=o\left(\epsilon^{k}\right)
$$

as $\epsilon \rightarrow 0+$ for every $k \geq 1$. 
Step 6.

Using the polynomial degeneracy hypothesis ((i) of Theorem VI.1), Step 5, Jensen's inequality, and Lemma 4.3 of ([BM4]), we obtain

$$
P\left(\int_{t_{0}}^{T}\{\hat{g}(u, x(u-r))\}^{2} d u<\epsilon\right)=o\left(\epsilon^{k}\right)
$$

as $\epsilon \rightarrow 0+$ for every $k \geq 1$.

Step $\%$.

Combining Steps 3 and 6 gives $P(\operatorname{det} C(T)<\epsilon)=o\left(\epsilon^{k}\right)$ as $\epsilon \rightarrow 0+$ for every $k \geq 1$. This implies that $C(T)^{-1}$ exists almost surely and $\operatorname{det} C(T)^{-1} \in \bigcap_{q=1}^{\infty} L^{q}(\Omega, \mathbf{R})$.

\section{Diffusions via sdde's.}

In this section, we will prove the following well-known existence theorem for classical diffusions $([\mathrm{GS}])$. The proof uses Caratheodory approximations by small delays:

\section{Theorem VI.4.}

Let $h: \mathbf{R}^{d} \rightarrow \mathbf{R}^{d}, g: \mathbf{R}^{d} \rightarrow \mathbf{R}^{d \times m}$ be globally Lipschitz, and $W$ be $m$-dimensional Brownian motion on a filtered probability space $\left(\Omega, \mathcal{F},\left(\mathcal{F}_{t}\right)_{t \geq 0}, P\right)$. Suppose $x_{0} \in \mathbf{R}^{d}$. Then the sode

$$
\left.\begin{array}{rl}
d x(t) & =h(x(t)) d t+g(x(t)) d W(t), \quad t>0 \\
x(0) & =x_{0}
\end{array}\right\}
$$

has an $\left(\mathcal{F}_{t}\right)_{t \geq 0}$-adapted solution with continuous sample paths.

Proof. ([BM5])

For simplicity assume that $h \equiv 0$ and $d=m=1$.

Fix $0<T<\infty$. Define the sequence of processes $\left\{x^{k}\right\}_{k=1}^{\infty}$ by

$$
x^{k}(t)=\left\{\begin{array}{l}
x_{0}+\int_{0}^{t} g\left[x^{k}(u-1 / k)\right] d W(u), \quad 0 \leq t \leq T \\
x_{0}, \quad-\frac{1}{k} \leq t \leq 0
\end{array}\right.
$$

for each integer $k \geq 1$. Note that each $x^{k}$ exists, is $\left(\mathcal{F}_{t}\right)_{t \geq 0}$-adapted and continuous. 
Step 1.

Each $x^{k}:[0, \infty) \rightarrow L^{2}(\Omega, \mathbf{R})$ is $\left(\frac{1}{2}\right)$-Hölder continuous with Hölder constant independent of $k$. To prove this, observe first that by $(*)$ and the linear growth property of $g$, there is a positive constant $K$ independent of $k$ and such that

$$
E \sup _{0 \leq t \leq T}\left(\left|x^{k}(t)\right|^{2}+\left|g\left(x^{k}(t)\right)\right|^{2}\right) \leq K
$$

for all $k \geq 1$. Then $E\left[x^{k}(t)-x^{k}(s)\right]^{2} \leq K(t-s)$ for all $t, s \in[0, T]$.

Step 2.

For each $t \geq 0, x^{k}(t)$ converges to a limit $x(t)$ in $L^{2}(\Omega, \mathbf{R})$.

Let $L$ be the Lipschitz constant for $g$. Then for $l>k$ and $0 \leq t \leq T$, we have

$$
\begin{aligned}
E\left[x^{l}(t)-x^{k}(t)\right]^{2} & =E\left\{\int_{0}^{t}\left[g\left(x^{l}(u-1 / l)\right)-g\left(x^{k}(u-1 / k)\right)\right] d W(u)\right\}^{2} \\
& \leq L^{2} \int_{0}^{t} E\left[x^{l}(u-1 / l)-x^{k}(u-1 / k)\right]^{2} d u \\
& \leq 2 L^{2} \int_{0}^{t} E\left[x^{l}(u-1 / l)-x^{k}(u-1 / l)\right]^{2} d u \\
& \quad+2 L^{2} \int_{0}^{t} E\left[x^{k}(u-1 / l)-x^{k}(u-1 / k)\right]^{2} d u \\
& \leq 2 L^{2} \int_{-(1 / l)}^{t-(1 / l)} E\left[x^{l}(u)-x^{k}(u)\right]^{2} d u+2 K L^{2} t\left(\frac{1}{k}-\frac{1}{l}\right) \\
& \leq 2 L^{2} \int_{0}^{t} E\left[x^{l}(u)-x^{k}(u)\right]^{2} d u+2 T K L^{2}\left(\frac{1}{k}-\frac{1}{l}\right)
\end{aligned}
$$

by Step 1. Thus, by Gronwall's lemma,

$$
E\left[x^{l}(t)-x^{k}(t)\right]^{2} \leq 2 T K L^{2}\left(\frac{1}{k}-\frac{1}{l}\right) e^{2 L^{2} t}, \quad 0 \leq t \leq T .
$$

Therefore the sequence $\left\{x^{k}(t)\right\}_{k=1}^{\infty}$ converges in $L^{2}(\Omega, \mathbf{R})$ to $x(t)$. Also

$$
E\left[x(t)-x^{k}(t)\right]^{2} \leq \frac{2 T K L^{2}}{k} e^{2 L^{2} t}, \quad 0 \leq t \leq T
$$


Step 3.

To show that the process $x$ satisfies the sode (XXI) (for $h \equiv 0$ ), simply take limits as $k \rightarrow \infty$ in both sides of $(*)$. The left-hand side of $(*)$ converges to $x(t)$ in $L^{2}$. Furthermore, $x$ is $\left(\mathcal{F}_{t}\right)_{t \geq 0}$-adapted, since each $x^{k}$ is. Also

$$
\begin{aligned}
E\left\{\int_{0}^{t}\left[g\left(x^{k}(u-1 / k)\right)-g(x(u))\right] d W(u)\right\}^{2} \\
\quad \leq L^{2} \int_{0}^{t} E\left[x^{k}(u-1 / k)-x(u)\right]^{2} d u \\
\quad \leq 2 L^{2} \int_{0}^{t} E\left[x^{k}(u-1 / k)-x^{k}(u)\right]^{2} d u+2 L^{2} \int_{0}^{t} E\left[x^{k}(u)-x(u)\right]^{2} d u \\
\quad \leq \frac{2 L^{2} K t}{k}+\frac{4 L^{4} K T}{k} \int_{0}^{t} e^{2 L^{2} u} d u \\
\quad \leq \frac{2 K L^{2}}{k}\left[t+T\left(e^{2 L^{2} t}-1\right)\right], \quad 0 \leq t \leq T
\end{aligned}
$$

From the above inequality, it follows that the right-hand side of $\left(^{*}\right)$ converges in $L^{2}$ to $x_{0}+\int_{0}^{t} g(x(u)) d W(u)$ as $k \rightarrow \infty$. Therefore $x$ satisfies the sode (XXI). Since the Itô integral has an almost surely continuous modification, it follows from Doob's inequality that $x$ also has such a modification.

\section{Affine sfde's. A simple model of population growth.}

Recall the following simple population growth model introduced in Chapter I (Example 2):

$$
d x(t)=\{-\alpha x(t)+\beta x(t-r)\} d t+\sigma d W(t), \quad t>0,
$$

for a large population $x(t)$ with constant birth rate $\beta>0$ (per capita), constant death rate $\alpha>0$ (per capita), development period $r$ and a migration rate given by white noise with variance $\sigma$.

To determine the stability and growth rates of the population, we will consider the more general affine system :

$$
\begin{aligned}
d x(t) & =\left\{\int_{[-r, 0]} \mu(d s) x(t+s)\right\} d t+d Q(t), \quad t>0 \\
x_{0} & =\eta \in D:=D\left([-r, 0], \mathbf{R}^{d}\right) .
\end{aligned}
$$

In $(\mathrm{X}), D$ is the space of all cadlag paths $[-r, 0] \rightarrow \mathbf{R}^{d}$ with the sup norm $\|\cdot\|_{\infty}, \mu$ is a finite Borel measure on $[-r, 0]$, and $Q$ is a cadlag $\mathbf{R}^{d}$-valued process. 
Theorem VI.5. ([MS3])

Let $A^{H}: D\left(A^{H}\right) \subset D \rightarrow D$ be the generator of the homogeneous equation

$$
\left.\begin{array}{rl}
d x(t) & =\left\{\int_{[-r, 0]} \mu(d s) x(t+s)\right\} d t, \quad t>0 \\
x_{0} & =\eta \in D .
\end{array}\right\}
$$

Let $\left\{\beta_{i}: i \geq 1\right\}$ be the set of all real parts of the spectrum of $A^{H}$. Then the Lyapunov spectrum of $(X)$ coincides with $\left\{\beta_{i}: i \geq 1\right\}$ together with possibly $-\infty$.

We now consider the hyperbolic case when $\beta_{i} \neq 0$ for all $i \geq 1$. In this case, the following result establishes the existence of a hyperbolic splitting along a unique stationary solution of (X) ([MS3], Theorem 20) .

Theorem VI.6. ([MS3])

Suppose that $Q$ is cadlag and has stationary increments. Assume that the characteristic equation

$$
\operatorname{det}\left(\lambda I-\int_{[-r, 0]} e^{\lambda s} \mu(d s)\right)=0
$$

has no roots on the imaginary axis; i.e., $\beta_{i} \neq 0$ for all $i \geq 1$. Suppose also that

$$
\limsup _{t \rightarrow \pm \infty} \frac{1}{|t|} \log |Q(t)|<\left|\beta_{i}\right| \quad \text { a.s. }
$$

for all $i \geq 1$. Then there is a unique D-valued random variable $\eta_{\infty}$ such that the trajectory $\left\{x_{t}^{\eta_{\infty}}: t \geq 0\right\}$ of $(X)$ is a D-valued stationary process. The random variable $\eta_{\infty}$ is measurable with respect to the $\sigma$-algebra generated by $\{Q(t): t \in \mathbf{R}\}$. Furthermore, let $\beta_{1}>\beta_{2}>\cdots$, be an ordering of the Lyapunov spectrum of $(X)$. Let $m$ be such that $\beta_{m}>0$ and $\beta_{m+1}<0$. Then there exists a decreasing sequence of finite-codimensional subspaces $\left\{E_{i}: i \geq 1\right\}$ of $D$ such that

$$
\lim _{t \rightarrow \infty} \frac{1}{t} \log \left\|x_{t}(\omega)\right\|_{\infty}=\beta_{i}, \quad i \geq 1
$$

if $x_{0}(\omega) \in \eta_{\infty}+E_{i-1} \backslash E_{i}, 1 \leq i \leq m$, and

$$
\varlimsup_{t \rightarrow \infty} \frac{1}{t} \log \left\|x_{t}(\omega)\right\|_{\infty} \leq \beta_{m+1}
$$

if $x_{0}(\omega) \in \eta_{\infty}+E_{m}$.

Results on the existence of $p$-th moment Lyapunov exponents appear in ([MS3]). Under a mild non-degeneracy condition on the stationary solution, one gets the existence 
of only one $p$-th moment exponent $\left(=p \beta_{1}\right)$ which is independent of all random (possibly anticipating) initial conditions in $D$. This result is in agreement with the affine linear (finite-dimensional) nondelay case $(r=0)$ ([AOP], [B], $[\mathrm{AKO}])$.

\section{Problem.}

Under what conditions on the parameters $\alpha, \beta$ does (II) have a stationary solution?

We conclude this section by noting the following interesting fact:

The affine hereditary system (X) may be viewed as a finite-dimensional stochastic perturbation of the associated infinitely degenerate deterministic homogeneous system $\left(X^{\prime}\right)$ with countably many Lyapunov exponents. However, these finite-dimensional perturbations provide noise that is generically rich enough to account for a single $p$-th moment Lyapunov exponent in the affine stochastic system $(\mathrm{X})$.

\section{Random delays.}

Sfde's with random delays are discussed in the monograph ([M1], pp. 167-186). In [M1], the delays are allowed to be random, independent of the noise and essentially bounded. The Markov property fails, but one gets a measure-valued process with random Markov transition measures on the state space $C$.

\section{Infinite delays. Stationary solutions.}

For a discussion of these issues, the reader may consult the pioneering work of Itô and Nisio ([IN], pp. 1-75). The main results in [IN] are summarized in [M1], pp. 230-233. For sfde's with fading memory, see the works of Mizel and Trutzer [MT], and Marcus and Mizel [MM]. 


\section{Bibliography}

[A] Ahmed, T.A., M.Sc. Thesis, University of Khartoum, (1983).

[AKO] Arnold, L., Kliemann, W. and Oeljeklaus, E. Lyapunov exponents of linear stochastic systems, in Lyapunov Exponents, Springer Lecture Notes in Mathematics, 1186 (1989), 85-125.

[AOP] Arnold, L. Oeljeklaus, E. and Pardoux, E., Almost sure and moment stability for linear Itô equations, in Lyapunov Exponents, Springer Lecture Notes in Mathematics, 1186 (ed. L. Arnold and V. Wihstutz) (1986), 129-159.

[B] Baxendale, P. H., Moment stability and large deviations for linear stochastic differential equations, in Ikeda, N. (ed.) Proceedings of the Taniguchi Symposium on Probabilistic Methods in Mathematical Physics, Katata and Kyoto (1985), 31-54, Tokyo: Kinokuniya (1987).

[BW] Bailey, H. R. and Williams, M. Z., Some results on the differential difference equation $x^{\prime}(t)=\sum_{i=0}^{N} A_{i} x\left(t-T_{i}\right)$, J. Math. Anal. Appl. 15 (1966), 569-587.

[B] Bell, D. R., Degenerate Stochastic Differential Equations and Hypoellipticity, Pitman Monographs and Surveys in Pure and Applied Mathematics, Vol. 79, Longman, Essex, 1995.

[BM1] Bell, D. R. and Mohammed, S.-E. A., The Malliavin calculus and stochastic delay equations, J. Funct. Anal. 99, No. 1 (1991), 75-99.

[BM2] Bell, D.R. and Mohammed, S.-E. A., An extension of Hörmander's theorem for infinitely degenerate parabolic operators, Duke Math. Journal 78 (1995), no. 3, 453-475.

[BM3] Bell, D. R. and Mohammed, S.-E. A., Opérateurs paraboliques hypoelliptiques avec dégénérescences exponentielles, C.R. Acad. Sci. Paris, t. 317, Série I, (1993), 10591064.

[BM4 Bell, D. R., and Mohammed, S.-E. A., Smooth densities for degenerate stochastic delay equations with hereditary drift, Ann. Prob. 23, no. 4, 1875-1894, 1995.

[BM5] Bell, D. R. and Mohammed, S.-E. A., On the solution of stochastic ordinary differential equations via small delays, Stochastics Stochastics Rep. 28 (1989), no. 4, 293-299 
[CJPS] Çinlar, E., Jacod, J., Protter, P. and Sharpe, M., Semimartingales and Markov processes, Z. Wahrsch. Verw. Gebiete, 54 (1980), 161-219.

[DoD] Doleans-Dade, C., On the existence and unicity of solutions of stochastic integral equations, Z. Wahrsch. Verw. Gebiete, 36 (1976), 93-101.

[Do] Doss, H., Liens entre équations différentielles stochastiques et ordinairés, Ann. Inst. Henri Poincaré, Vol. XIII, no. 2 (1977), 99-125.

[Du] Dudley, R. M., The sizes of compact subsets of Hilbert space and continuity of Gaussian processes, J. Functional Analysis,1, (1967), 290-330.

[Dy] Dynkin, E. B., Markov Processes, Vols I, II, Springer-Verlag, Berlin (1965).

[DM] Delfour, M. C. and Mitter, S. K., Hereditary differential systems with constant delays. I. General case. J. Differential Equations 12 (1972), 213-235; erratum, ibid. 14 (1973), 397.

[DS] Dunford and Schwartz, Linear Operators, Part I: General Theory, Interscience Publishers, New York (1958).

[FS] Flandoli, F. and Schaumlöffel, K.-U., Stochastic parabolic equations in bounded domains: Random evolution operator and Lyapunov exponents, Stochastics and Stochastic Reports, 29, 4 (1990), 461-485.

[FT] Frampton, J. and Tromba, A. J. On the classification of spaces of Hölder continuous functions, J. Functional Analysis, 10 (1972), 336-345

[Fr] Friedman, A., Stochastic Differential Equations and Applications, Vol. 1, Academic Press, New York, San Francisco, London (1975).

[GRR] Garsia, A. M., Rodemich, E. and Rumsey, H., Jr., A real variable lemma and the continuity of paths of some Gaussian processes, Indiana Univ. Math. J., 20 (1970/1971), 565-578.

[GS] Gihman, I. I. and Skorohod, A. V., Stochastic Differential Equations, SpringerVerlag, New York (1973).

[H] Hale, J. K., Theory of Functional Differential Equations, Springer-Verlag, New York, Heidelberg, Berlin, (1977). 
[Ha] Has'minskii, R. Z., Stochastic Stability of Differential Equations, Sijthoff and Noordhoff (1980).

[IN] Itô, K. and Nisio, M., On stationary solutions of a stochastic differential equation, J. Math. Kyoto University, 4-1 (1964), 1-75.

[Kub] Kubo, R., The fluctuation-dissipation theorem and Brownian motion, in Many-Body Theory edited by R. Kubo, Syokabo and Benjamin (1966), 1-16.

[Kun] Kunita, H., Stochastic Flows and Stochastic Differential Equations, Cambridge University Press, Cambridge, New York, Melbourne, Sydney (1990).

[Kus] Kushner, H. J., On the stability of processes defined by stochastic differentialdifference equations, J. Differential Equations, 4, (1968), 424-443.

[KN] Kolmanovskii, V. B. and Nosov, V. R., Stability of Functional Differential Equations, Academic Press, London, Orlando (1986).

[KS1] Kusuoka, S., and Stroock, D. W., Applications of the Malliavin calculus, I, Taniguchi Sympos. SA Katata (1982), 271-306.

[KS2] Kusuoka, S., and Stroock, D. W., Applications of the Malliavin calculus, II, J. Fac. Sci. Univ. Tokyo, Sect. 1 A Math., 32, No. 1 (1985), 1-76.

[LT] Lenhart, S. M.; Travis, C. C., Global stability of a biological model with time delay, Proc. Amer. Math. Soc., 96, no. 1, (1986),75-78.

[LS] Lipster, R. S., and Shiryayev, A. N. Statistics of Random Processes , Springer, Berlin (1977).

[Ma] Mao, X.R., Exponential Stability of Stochastic Differential Equations, Pure and Applied Mathematics, Marcel Dekker, New York-Basel-Hong Kong (1994).

[Met] Metivier, M. Semimartingales, a Course on Stochastic Processes, Walter de Gruyter, Berlin-New York (1982).

[M1] Mohammed, S.-E.A. Stochastic Functional Differential Equations, Research Notes in Mathematics, 99, Pitman Advanced Publishing Program, Boston, London, Melbourne (1984).

[M2] Mohammed, S.-E. A., Non-linear flows for linear stochastic delay equations, Stochastics, 17, 3 (1986), 207-212. 
[M3] Mohammed, S.-E. A., The Lyapunov spectrum and stable manifolds for stochastic linear delay equations, Stochastics and Stochastic Reports, 29 (1990), 89-131.

[M4] Mohammed, S.-E. A., Lyapunov exponents and stochastic flows of linear and affine hereditary systems, (Survey article), in Diffusion Processes and Related Problems in Analysis, Volume II, edited by M., Pinsky and V., Wihstutz, Birkhäuser (1992), 141-169.

[MM] Marcus, M., and Mizel, V. J., Stochastic functional differential equations modeling materials with selective recall, Stochastics 25 (1988), no. 4, 195-232

[MP] Metivier, M. and Pellaumail, J. Stochastic Integration, Academic Press, LondonNew York (1980).

[MT] Mizel, V. J. and Trutzer, V., Stochastic hereditary equations: existence and asymptotic stability, Journal of Integral Equations 7 (1984), 1-72.

[MS1] Mohammed, S.-E. A. and Scheutzow, M. K. R., Lyapunov exponents of linear stochastic functional differential equations driven by semimartingales. Part I: The multiplicative ergodic theory, Ann. Inst. Henri Poincaré, Probabilités et Statistiques, vol. 32, (1996), 69-105.

[MS2] Mohammed, S.-E. A. and Scheutzow, M. K. R., Lyapunov exponents of linear stochastic functional differential equations driven by semimartingales. Part II: Examples and case studies, to appear in The Annals of Probability.

[MS3] Mohammed, S.-E. A. and Scheutzow, M. K. R., Lyapunov exponents and stationary solutions for affine stochastic delay equations, Stochastics and Stochastic Reports, Vol. 29, No. 2 (1990), 259-283

[PW1] Pardoux, E. and Wihstutz, V., Lyapunov exponent and rotation number of twodimensional stochastic systems with small diffusion, SIAM J. Applied Math., 48, (1988), 442-457.

[PW2] Pinsky, M. and Wihstutz, V., Lyapunov exponents of nilpotent Itô systems, Stochastics, 25, (1988), 43-57.

[P1] Protter, Ph. E., On the existence, uniqueness and explosion of solutions of systems of stochastic integral equations, Ann. Prob. vol. 5, (1977), 243-261. 
[P2] Protter, Ph. E., Semimartingales and measure-preserving flows, Ann. Inst. Henri Poincaré, Probabilités et Statistiques, vol. 22, (1986), 127-147.

[R] Ruelle, D., Characteristic exponents and invariant manifolds in Hilbert space, Annals of Mathematics, 115 (1982), 243-290.

[S] Scheutzow, M. K. R., Stationary and Periodic Stochastic Differential Systems: A study of Qualitative Changes with Respect to the Noise Level and Asymptotics, Habiltationsschrift, University of Kaiserslautern, Germany (1988).

[Sc] Schwartz, L., Radon Measures on Arbitrary Topological Spaces and Cylindrical measures, Tata Institute of Fundamental Research, Oxford University Press, (1973).

[Sk] Skorohod, A. V., Random Linear Operators, D. Reidel Publishing Company (1984).

[St] Stroock, D. W., The Malliavin calculus, a functional analytic approach, J. Funct. Anal. 44 (1981), 212-257.

[Su] Sussman, H. J., On the gap between deterministic and stochastic ordinary differential equations, Ann. Prob., 6, 1 (1978), 19-41.

[SM1] de Sam Lazaro, J. and Meyer, P. A., Méthodes de martingales et théorie des flots , Z. Wahrsch. Verw. Gebiete, Vol. 18, (1971), 116-140.

[SM2] de Sam Lazaro, J. and Meyer, P. A., Questions de théorie des flots, Seminaire de Probab. IX, Springer Lecture Notes in Mathematics, 465 (1975), 1-96.

[Tr] Tromba, A. J., On the isometries of spaces of Hölder continuous functions, Studia Math. 57 (1976), no. 3, 199-208. 